CENTRO UNIVERSITÁRIO FEI

MARCELO MACHADO AOYAMA

DISTORÇÃO HARMÔNICA EM MOSFETS IMPLEMENTADOS COM OS ESTILOS DE LEIAUTE DO TIPO DIAMANTE HÍBRIDO E CONVENCIONAIS

São Bernardo do Campo

2019 


\section{DISTORÇÃO HARMÔNICA EM MOSFETS IMPLEMENTADOS COM OS ESTILOS DE LEIAUTE DO TIPO DIAMANTE HÍBRIDO E CONVENCIONAIS}

Dissertação apresentada ao Centro Universitário FEI como parte dos requisitos necessários para obtenção do título de Mestre em Engenharia Elétrica. Orientado pelo Prof. Dr. Salvador Pinillos Gimenez.

São Bernardo do Campo 
Machado Aoyama, Marcelo.

DISTORÇÃO HARMÔNICA EM MOSFETs IMPLEMENTADOS COM OS ESTILOS DE LEIAUTE DO TIPO DIAMANTE HÍBRIDO E CONVENCIONAIS / Marcelo Machado Aoyama. São Bernardo do Campo, 2019.

90 p. : il.

Dissertação - Centro Universitário FEI.

Orientador: Prof. Dr. Salvador Pinillos Gimenez.

1. Geometria não-convencional de porta para MOSFETs. 2. MOSFET do tipo Diamante Híbrido. 3. Distorção Harmônica. 4. Caracterização elétrica experimental. I. Pinillos Gimenez, Salvador, orient. II. Título. 
Título do Trabalho: Distorção harmônica entre os mosfets implementados com os estilos de leiaute do tipo diamante híbrido e convencionais.

Área de Concentração: Nanoeletrônica e Circuitos Integrados

Orientador: Prof. Dr. Salvador Pinillos Gimenez

Data da realização da defesa: 11/11/2019

\section{ORIGINAL ASSINADA}

Avaliação da Banca Examinadora:

São Bernardo do Campo, / /

\section{MEMBROS DA BANCA EXAMINADORA}

Prof. Dr. Salvador Pinillos Gimenez

Ass.:

Profa Dra Michelly de Souza

Ass.:

Prof. Dr. Renan Trevisoli Doria

Ass.:

A Banca Julgadora acima-assinada atribuiu ao aluno o seguinte resultado:

$\begin{array}{ll}\text { APROVADO } \bigotimes & \text { REPROVADO }\end{array}$

VERSÃO FINAL DA DISSERTAC̄̃̃O

APROVO A VERSÃO FINAL DA DISSERTAÇÃO EM QUE FORAM INCLUÍDAS AS RECOMENDAÇÕES DA BANCA EXAMINADORA
Aprovação do Coordenador do Programa de Pós-graduação

Prof. Dr. Carlos Eduardo Thomaz 
A Deus que sempre guiou e guia os meus passos, aos meus pais, Marcelo e Iolanda, que dignamente me apresentaram a importância do estudo, da perseverança, da fé e o caminho da honestidade e à minha família e ao meu irmão Eduardo que sempre me apoiou nessa caminhada da vida. 


\section{AGRADECIMENTOS}

Primeiramente a Deus que permitiu que tudo ao longo da minha vida fosse possível, e não somente nestes anos como estudante de mestrado, mas em todos os momentos da vida que é o maior mestre que alguém pode conhecer.

Ao Centro universitário FEI, seu corpo docente, direção e administração que me oportunizaram a janela que hoje vislumbro um horizonte superior, eivado pela acendrada confiança no mérito e ética.

Ao Prof. Dr. Salvador Pinillos Gimenez, o meu reconhecimento pela oportunidade de realizar este trabalho ao lado de alguém que emana sabedoria; meu respeito e admiração pela sua serenidade, capacidade de análise do perfil de seus alunos, e pelo seu Dom no ensino da Ciência, inibindo sempre a vaidade em prol da simplicidade e eficiência.

Também gostaria de agradecer aos professores doutores Rodrigo Doria, Milene Galeti, Michelly de Souza e Renan Trevisoli que ajudaram no meu desenvolvimento não só como mestre, todavia, também, como pessoa.

O presente trabalho foi realizado com apoio da Coordenação de Aperfeiçoamento de Pessoal de Nível Superior - Brasil (CAPES) - Código de Financiamento 001. E também com o apoio do Conselho Nacional de Desenvolvimento Científico e Tecnológico (CNPq). 
"Dê-me, Senhor, agudeza para entender, capacidade para reter, método e faculdade para aprender, sutileza para interpretar, graça e abundância para falar, acerto ao começar, direção ao progredir e perfeição ao concluir." São Tomas de Aquino 


\section{RESUMO}

$\mathrm{Na}$ atualidade, há diferentes frentes de pesquisa (uso de novas estruturas de transistores, novos materiais, etc.) quer nas instituições de ensino, quer nos centros de pesquisa, visando a redução das dimensões do Transistor de Efeito de Campo Metal-Óxido-Semicondutor (MOSFET), para que, desta forma, possa continuar a atender a chamada Lei de Moore. Recentemente, foi proposta uma inovadora abordagem que não adiciona custos aos atuais processos de fabricação de circuitos integrados (CIs) MOS Complementar planares, chamada de MOSFET com leiaute de portas não convencionais, que são capazes de potencializar o seu desempenho elétrico em aplicações analógicas em relação ao MOSFET de geometria de porta retangular equivalente. Esta dissertação de mestrado visa realizar um estudo experimental comparativo do comportamento da distorção harmônica total normalizada pelo ganho de tensão (THD/Av) no MOSFET com leiaute de porta do tipo Diamante Híbrido (Composto pela associação paralela de 3 MOSFETs: ao centro um MOSFET do tipo Diamante e dois MOSFETs de porta retangular) em relação ao MOSFET convencional de geometria de porta retangular (CM), por se tratar de uma figura de mérito de grande importância em aplicações analógicas. O MOSFET do tipo Diamante Híbrido (HDM) foi desenvolvido devido às limitações do processo de fabricação de CIs mais sofisticados que não permitem que a região de porta seja definida de forma ortogonal à da região ativa do transistor. Os MOSFETs utilizados neste projeto de pesquisa foram fabricados com processo comercial de fabricação de CIs CMOS convencional (Bulk) de 0,18 $\mu \mathrm{m}$ da TSMC, via programa universitário Mini@sic do IMEC. O Método da Função Integral foi usado para realizar o estudo da THD/Av dos MOSFETs. Esse método usa somente a curva característica de corrente contínua do MOSFET operando na região de saturação (essa curva é a de corrente entre o dreno e a fonte em função da tensão entre a porta e a fonte). Um dos principais resultados obtidos mostra que o HDM com um ângulo alfa $(\alpha)$ igual a $45^{\circ}$ é capaz de reduzir a THD/Av cerca de $6,4 \mathrm{~dB}$ para uma sobretensão de porta igual a $600 \mathrm{mV}$ quando comparado ao CM, isso ocorre porque o MOSFET do tipo Diamante Híbrido com ângulo $\alpha$ igual a $45^{\circ}$ apresentou um ganho de tensão intrísico maior que à do $\mathrm{CM}$, visto que a THD/Av é fortemente dependente do ganho de tensão desses dispositivos.

Palavras-chave: Geometria não-convencional de porta para MOSFETs. Estilo de leiaute do tipo Diamante. MOSFET do tipo Diamante Híbrido. Linearidade. Distorção Harmônica. Caracterização elétrica experimental. Distorção Harmônica Total. CI. 


\begin{abstract}
Nowadays there are different researches fronts (use of new transistor structures, new materials, etc.) both in educational institutions and research centers, that aim to reduce the size of the Metal-Oxide-Semiconductor Field Effect Transistor (MOSFET), then to keep the socalled Moore Law. An innovative approach has been recently proposed that does not add additional costs to the current manufacturing processes of Planar Complementary MOS Integrated Circuits (ICs), called unconventional gate layout MOSFETs, which are able to enhance their electrical performance in analog applications over the equivalent rectangular gate geometry MOSFET. This master thesis aims to conduct a comparative experimental study of the behavior of the total harmonic distortion normalized by the voltage gain (THD/Av) in the Hybrid Diamond type gate layout MOSFET (Composed by the parallel association of 3 MOSFETs: in the center a MOSFET of Diamond type and two rectangular gate MOSFETs) compared to the conventional rectangular gate geometry MOSFET (CM), as it is a very important figure of merit in analog applications. Hybrid Diamond MOSFET (HDM) was developed due to the limitations of the manufacturing process of more sophisticated ICs that do not allow the gate region to be defined orthogonally to the active transistor region. The MOSFETs used in this research project were manufactured using commercial process TSMC's $0.18 \mu \mathrm{m}$ CMOS Bulk via IMEC's Mini @ sic university program. The Integral Function Method was used to perform the THD/Av study of MOSFETs. This method uses only the direct current characteristic curve of the MOSFET operating in the saturation region (this curve is the current between the drain and the source as a function of the voltage between the gate and the source). One of the main results obtained by this research project shows that HDM with an alpha $(\alpha)$ angle of $45^{\circ}$ is able to reduce THD/Av by about $6.4 \mathrm{~dB}$ for a gate overvoltage of $600 \mathrm{mV}$ when Compared to CM, this is because the Hybrid Diamond MOSFET with angle $\alpha$ equal to $45^{\circ}$ had a higher intrinsic voltage gain than CM, since THD/Av is strongly dependent on the voltage gain of these devices.
\end{abstract}

Key words: Non-conventional gate geometry for MOSFETs. Layout style of Diamond type. Hybrid Diamond-type MOSFET. Linearity. Harmonic Distortion. Experimental electrical characterization. Total Harmonic Distortion. IC. 


\section{LISTA DE SÍMBOLOS}

\begin{tabular}{|c|c|}
\hline $\mathrm{A}_{\mathrm{G}}$ & Área de porta $\left[\mu \mathrm{m}^{2}\right]$ \\
\hline Av & Ganho de tensão intrínseco do transistor [V/V] \\
\hline $\mathrm{B}$ & Maior comprimento de canal do MOSFET dos tipos Diamante e Octo $[\mu \mathrm{m}]$ \\
\hline $\mathrm{b}$ & Menor comprimento de canal do MOSFET dos tipos Diamante e Octo $[\mu \mathrm{m}]$ \\
\hline $\mathrm{C}$ & Capacitância $[\mathrm{F}]$ \\
\hline $\mathrm{C}_{0}$ & $\begin{array}{l}\text { Coeficiente de Fourier correspondente à amplitude do nível DC do sinal de } \\
\text { saída de um circuito elétrico }\end{array}$ \\
\hline $\mathrm{C}_{1}$ & $\begin{array}{l}\text { Coeficiente de Fourier correspondente à amplitude do sinal de frequência } \\
\text { fundamental na saída de um circuito elétrico }\end{array}$ \\
\hline $\mathrm{C}_{2}$ & $\begin{array}{l}\text { Coeficiente de Fourier correspondente à amplitude do segundo harmônico no } \\
\text { sinal de saída de um circuito elétrico }\end{array}$ \\
\hline $\mathrm{C}_{\mathrm{n}}$ & $\begin{array}{l}\text { Coeficiente de Fourier correspondente à amplitude do harmônico de ordem n } \\
\text { no sinal de saída }\end{array}$ \\
\hline $\mathrm{C}_{\mathrm{L}}$ & Capacitor de carga $[\mathrm{F}]$ \\
\hline Cox & Capacitância do óxido de porta por unidade de área $\left[\mathrm{F} / \mathrm{cm}^{2}\right]$ \\
\hline dMÁx & Largura máxima da região de depleção $[\mathrm{cm}]$ \\
\hline$\vec{\varepsilon}_{/ /}$ & $\begin{array}{l}\text { Campo elétrico longitudinal resultante do MOSFETs do tipo Diamante } \\
{[\mathrm{kV} / \mathrm{cm}]}\end{array}$ \\
\hline$\vec{\varepsilon}_{/ / 1}$ & $\begin{array}{l}\text { Componente } 1 \text { do campo elétrico longitudinal do MOSFETs do tipo Diamante } \\
{[\mathrm{kV} / \mathrm{cm}]}\end{array}$ \\
\hline$\vec{\varepsilon}_{/ / 2}$ & $\begin{array}{l}\text { Componente } 2 \text { do campo elétrico longitudinal do MOSFETs do tipo Diamante } \\
{[\mathrm{kV} / \mathrm{cm}]}\end{array}$ \\
\hline $\mathrm{f}$ & Frequência $[\mathrm{Hz}]$ \\
\hline $\mathrm{f}_{\mathrm{T}}$ & Frequência de ganho de tensão unitário $[\mathrm{Hz}]$ \\
\hline GI & $\begin{array}{l}\text { Ganho de IDS/(W/L) do MOSFET do tipo Diamante Híbrido em relação àquela } \\
\text { de MOSFET com leiaute de porta retangular }\end{array}$ \\
\hline GGI & $\begin{array}{l}\text { Ganho de } \mathrm{gm}_{\mathrm{m}} / \mathrm{IDS}_{\mathrm{DS}} \text { em função de IDS/(W/L) do MOSFET do tipo Diamante } \\
\text { Híbrido em relação ao do MOSFET com leiaute de porta retangular }\end{array}$ \\
\hline $\mathrm{GGV}_{\mathrm{GV}}$ & $\begin{array}{l}\text { Ganho de } \mathrm{g}_{\mathrm{m}} / \mathrm{IDS}_{\mathrm{DS}} \text { em função de } \mathrm{V}_{\mathrm{GT}} \text { do MOSFET do tipo Diamante Híbrido em } \\
\text { relação ao do MOSFET com leiaute de porta retangular }\end{array}$ \\
\hline$g_{D}$ & Condutância de dreno ou condutância de saída [S] \\
\hline
\end{tabular}




\begin{tabular}{|c|c|}
\hline $\mathrm{g}_{\mathrm{m}}$ & Transcondutância do MOSFET [S] \\
\hline$g_{\text {míx }}$ & Transcondutância máxima do MOSFET [S] \\
\hline \multirow[t]{2}{*}{$\mathrm{g}_{\mathrm{m}} / \mathrm{IDS}$} & Razão entre a transcondutância e a corrente entre o dreno e a fonte do \\
\hline & $\operatorname{MOSFET}\left[\mathrm{V}^{-1}\right]$ \\
\hline HD0 & Distorção harmônica correspondente à parcela DC do sinal de entrada [dB] \\
\hline HD2 & Distorção harmônica referente ao harmônico de segunda ordem [dB] \\
\hline HD3 & Distorção harmônica referente ao harmônico de terceira ordem [dB] \\
\hline HDn & Distorção harmônica referente ao harmônico de enésima ordem [dB] \\
\hline I & Corrente Elétrica $[\mathrm{A}]$ \\
\hline IDS & Corrente entre dreno e fonte $[\mathrm{A}]$ \\
\hline IDSsat & Corrente entre dreno e fonte de saturação [A] \\
\hline $\mathrm{I}_{\mathrm{G}}$ & Corrente de porta do MOSFET [A] \\
\hline ILEAK & Corrente de fuga do MOSFET [A] \\
\hline IOFF & Corrente de estado desligado entre dreno e fonte do MOSFET [A] \\
\hline ION & Corrente de estado ligado entre dreno e fonte do MOSFET [A] \\
\hline ION/IOFF & $\begin{array}{l}\text { Razão entre a corrente de estado ligado entre dreno e fonte e a corrente de } \\
\text { estado desligado entre dreno e fonte do MOSFET [A/A] }\end{array}$ \\
\hline $\mathrm{L}$ & Comprimento de canal do MOSFET $[\mu \mathrm{m}]$ \\
\hline $\mathrm{LD}$ & $\begin{array}{l}\text { Comprimento de canal de um MOSFET convencional que apresenta a mesma } \\
\text { área de porta que um MOSFET de leiaute de porta não convencional }[\mu \mathrm{m}]\end{array}$ \\
\hline Leff & Comprimento efetivo de canal do MOSFET $[\mu \mathrm{m}]$ \\
\hline $\mathrm{P}_{1}$ & Potência elétrica na frequência fundamental do sinal de saída [W] \\
\hline $\mathrm{P}_{2}$ & $\begin{array}{l}\text { Potência elétrica no segundo harmônico presente do sinal de saída de um } \\
\text { circuito elétrico }[\mathrm{W}]\end{array}$ \\
\hline $\mathrm{P}_{3}$ & $\begin{array}{l}\text { Potência elétrica no terceiro harmônico presente do sinal de saída de um } \\
\text { circuito elétrico }[\mathrm{W}]\end{array}$ \\
\hline $\mathrm{P}_{4}$ & $\begin{array}{l}\text { Potência elétrica no quarto harmônico presente do sinal de saída de um circuito } \\
\text { elétrico }[\mathrm{W}]\end{array}$ \\
\hline Pn & $\begin{array}{l}\text { Potência elétrica no enésimo harmônico presente do sinal de saída de um } \\
\text { circuito elétrico }[\mathrm{W}]\end{array}$ \\
\hline q & Carga elementar do elétron $[\mathrm{C}]$ \\
\hline $\mathrm{R}_{\mathrm{CH}}$ & Resistência de canal de um MOSFET $[\Omega]$ \\
\hline Rs & Resistência série de um MOSFET $[\Omega]$ \\
\hline RsD & Resistência total entre o dreno e a fonte do MOSFET $[\Omega]$ \\
\hline
\end{tabular}


RoN Resistência entre o dreno e a fonte de estado ligado do MOSFET [ $\Omega$ ] Inclinação de sublimiar do MOSFET [mV/década]

THD Distorção harmônica total [dB]

THD 0 Distorção harmônica total levando-se em conta o nível DC do sinal amplificado pelos MOSFETs [dB]

V Tensão Elétrica [V]

$\mathrm{V}_{0} \quad$ Nível de tensão DC entre porta e fonte [V]

$\mathrm{V}_{1} \quad$ Amplitude da tensão na frequência fundamental no sinal de saída de um circuito elétrico $[\mathrm{V}]$

$\mathrm{V}_{2} \quad$ Amplitude da tensão do segundo harmônico do sinal de saída de um circuito elétrico [V]

$\mathrm{V}_{3} \quad$ Amplitude da tensão do terceiro harmônico do sinal de saída de um circuito elétrico $[\mathrm{V}]$

V4 Amplitude da tensão do quarto harmônico do sinal de saída de um circuito elétrico $[\mathrm{V}]$

$\mathrm{Va}$ Amplitude máxima do sinal de entrada senoidal aplicado à entrada de um MOSFET [V]

VDD

VDS

Tensão positiva de alimentação de um inversor CMOS [V]

VEA

Tensão entre dreno e fonte [V]

VEA1

Tensão Early [V]

VEA2

Tensão Early determinada por uma dada sobretensão de porta 1 [V]

VEA3 Tensão Early determinada por uma dada sobretensão de porta 2 [V]

$\mathrm{V}_{\mathrm{FB}}$

$\mathrm{V}_{\mathrm{G}}$

$\mathrm{V}_{\mathrm{GS}}$

$\mathrm{V}_{\mathrm{GT}}$ Tensão Early determinada por uma dada sobretensão de porta 3 [V]

$\mathrm{V}_{\mathrm{GT} 1}$ Tensão de faixa plana [V]

VGT2 Tensão de porta [V] Tensão entre porta e fonte [V]

Sobretensão de porta [V] Sobretensão de porta de índice 1 [V]

Sobretensão de porta de índice 2 com valor maior que a sobretensão de porta 1 [V]

V Sobretensão de porta de índice 3 com valor maior que a sobretensão de porta 2 [V]

VIN Tensão de entrada de um MOSFET [V]

Vout $\quad$ Tensão de saída de um MOSFET [V] 
$\mathrm{V}_{\mathrm{TH}} \quad$ Tensão de limiar [V]

$\mathrm{X}_{0} \quad$ Ponto de polarização da tensão entre porta e fonte do MOSFET para o estudo da distorção harmônica utilizando o IFM [V]

W Largura de canal do MOSFET $[\mu \mathrm{m}]$

W/L Fator geométrico, ou razão de aspecto, do MOSFET

(W/L)HDM Fator geométrico, ou razão de aspecto, do MOSFET do tipo Diamante Híbrido

Xox $\quad$ Espessura do óxido de silício [nm]

$\alpha \quad$ Ângulo de abertura dos MOSFETs do tipo Diamante $\left[{ }^{\circ}\right]$

$\Delta \mathrm{IDS} \quad$ Variação de IDS [A]

$\Delta V_{D S} \quad$ Variação de VDS [V]

Eox Permissividade elétrica do óxido de silício [F/cm]

$\Phi_{\mathrm{F}} \quad$ Potencial de Fermi [V]

$\Phi_{\mathrm{MS}} \quad$ Diferença da função trabalho entre o metal e o semicondutor [V]

$\mu_{\mathrm{n}} \quad$ Mobilidade dos elétrons no silício $\left[\mathrm{cm}^{2} /(\mathrm{V} \cdot \mathrm{s})\right]$ 


\section{LISTA DE ABREVIATURAS}

AC Corrente elétrica alternada (Alternate Current)

AREA1 Área inferior do quadro normalizado pelo método da função integral

AREA2 Área superior do quadro normalizado pelo método da função integral

BBR Região de bico de pássaro (Bird's Beak Region)

CIs Circuitos Integrados (Integrated Circuit)

CM MOSFET convencional com leiaute de porta retangular (Conventional MOSFET)

$\mathrm{CM}_{1} \quad$ MOSFET convencional com leiaute de porta retangular presente na estrutura do MOSFET do tipo Diamante Híbrido

CMOS Metal-Óxido-Semicondutor Complementar (Complementary Metal-OxideSemiconductor)

D Contato de dreno (Drain Contact)

DC Corrente elétrica contínua (Direct Current)

DM MOSFET com leiaute de porta do tipo Diamante (geometria de porta retangular)

FET Transistor de Efeito de Campo (Field-Effect Transistor)

G Contato de porta (Gate Contact)

GAA Dispositivos silício sobre isolante de porta circundante (Gate-All-Around)

GC Canal gradual (Graded-Channel)

IFM Método da Função Integral (Integral Function Method)

JNT Transistors de nano fios sem junção (Junctionless Nanowire Transistors)

HD Distorção Harmônica (Harmonic Distortion)

HD2 Distorção Harmônica de Segunda Ordem (Second Order Harmonic Distortion)

HD3 Distorção Harmônica de Terceira Ordem (Third Order Harmonic Distortion)

HDn Distorção harmônica de ordem n (Harmonic Distortion n-order)

HDM MOSFET com leiaute de porta do tipo Diamante Híbrido (Hibrid Diamond MOSFET)

LEF $\quad$ Campo elétrico longitudinal (Longitudinal Electrical Field) 
LCE $\quad$ Efeito de canto longitudinal (Longitudinal Corner Effect)

LD Comprimento de canal do MOSFET convencional com leiaute de porta retangular que apresenta a mesma área de porta que um MOSFET do tipo Diamante

MOS Metal-Óxido-Semicondutor (Metal-Oxide-Semiconductor)

MOSFET Transistor de Efeito de Campo Metal-Óxido-Semicondutor (Metal-OxideSemiconductor Field-Effect Transistor)

MOSIS Metal-Oxide-Semiconductor Implementation Service

nMOSFET MOSFET do tipo $n$

og Ordem de grandeza

OTA Amplificador Operacional de Transcondutância (Operational

Transconductance Amplifier)

pMOSFET MOSFET do tipo $\mathrm{p}$

S

Contato de fonte (Source Contact)

$\mathrm{Si}$

Silício

SoCs Sistemas eletrônicos em um único circuito integrado (Systems-on-a-chip)

SOI Silício sobre Isolante (Silicon-on-Insulator)

$\mathrm{W}_{\mathrm{R}} \quad$ Largura de canal do MOSFET convencional com leiaute de porta retangular presente na estrutura do MOSFET do tipo Diamante Híbrido

WRR Largura de canal do MOSFET convencional com leiaute de porta retangular que tem a mesma área de porta que um MOSFET do tipo Diamante 


\section{LISTA DE ILUSTRAÇÕES}

Figura 1 - Fotografia de um MOSFET do tipo Diamante, do tipo n, implementado com tecnologia convencional (Bulk) de $350 \mathrm{~nm}$ da ON-Semiconductor.

Figura 2 - Fotografia de um MOSFET do tipo Octagonal, do tipo n, implementado com tecnologia SOI de $1 \mu \mathrm{m}$ da Universidade Católica de Louvain La Nueve (UCL), da Bélgica.13 Figura 3 - Fotografia de um MOSFET do tipo Elipsoidal, do tipo n, implementado com tecnologia Bulk de $350 \mathrm{~nm}$ da ON-Semiconductor.

Figura 4 - Leiaute simplificado de um MOSFET do tipo Diamante-Híbrido, do tipo n, de tecnologia Bulk.

Figura 5 - Secção transversal da estrutura de um MOSFET do tipo n construído com

tecnologia Bulk CMOS de CIs. 15

Figura 6 - Simbologias e as secções transversais dos MOSFETs.

Figura 7 - Gráfico da derivada da transcondutância de um nMOSFET em função da tensão de porta, para um VDS tendendo a zero (Neste caso $V_{D S}$ igual a $50 \mathrm{mV}$ ) utilizado para obter o valor de $\mathrm{V}_{\mathrm{TH}}$.

Figura 8 - Gráfico da transcondutância em função das variações da tensão entre a porta e a fonte para poder obter a transcondutância máxima.

Figura 9 - Exemplo de uma curva de $\mathrm{g}_{\mathrm{m}} / \mathrm{IDS}_{\mathrm{DS}}$ em função de IDS/(W/L) de um nMOSFET 20

Figura 10 - Ilustração esquemática da obtenção da VEA por meio da extrapolação da região linear da região de saturação da curva IDS em função de VDS para vários valores de VGS até

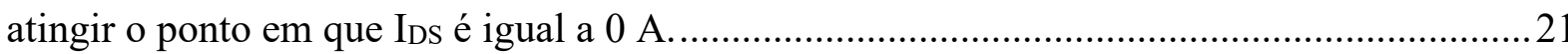

Figura 11 - Circuito amplificador de transcondutância de um único MOSFET.

Figura 12 - Efeito da distorção linear (a) e não-linear (b) sobre o espectro de f de um sistema eletrônico.

Figura 13 - Circuito utilizado para realizar a medida da distorção harmônica num nMOSFET (a) e a distorção da corrente de dreno devido a não-linearidade na curva IDs em função da VGS, para um determinado valor de VDS (b) quando é amplificado um sinal senoidal de amplitude Va e frequência f na entrada do MOSFET $\left(V_{G S}=V_{0}+V_{a} * \operatorname{sen}(\omega \cdot t)\right)$.

Figura 14 - Ilustração do processo de normalização de parte da curva característica IDs em função de $V_{\mathrm{GS}}$ que é dado pelo método IFM.

Figura 15 - Exemplo de uma curva característica IDs em função de $V_{G S}$ normalizada, em que são evidenciadas as áreas abaixo e acima da curva definido por $\mathrm{Y}=\mathrm{X}$. 
Figura 16 - Exemplo de uma curva característica normalizada do MOSFET que intercepta a bissetriz em um ponto qualquer e a correção feita através da função ys(x) pelo método IFM para determinação da distorção harmônica

Figura 17 - Vista superior simplificada do leiaute de um MOSFET do tipo Diamante mostrando as suas componentes do campo elétrico longitudinal, sua resultante e as características dimensionais.

Figura 18 - Representação física do MOSFET do tipo Diamante construído de infinitos MOSFETs com geometria de porta retangular associados em paralelo. 35

Figura 19 - Correspondente circuito elétrico equivalente de um DM. 36

Figura 20 - Linhas de campo elétrico longitudinais resultantes curvas entre as regiões de Dreno/Fonte e Canal do MOSFET do tipo Diamante (geometria de porta hexagonal) para um VDS maior do que $0 \mathrm{~V}$

Figura 21 - Vista superior do leiaute de um nMOSFET de geometria de porta hexagonal (DM) em paralelo com dois nMOSFETs com porta retangular $\left(\mathrm{CM}_{1}\right)$. 38 Figura 22 - Processo de transformação de um MOSFET convencional obtido de um projeto de CI CMOS analógico num HDM: Leiaute simplificado de um MOSFET convencional (a). MOSFET convencional híbrido implementado com um MOSFET retangular principal e dois MOSFETs secundários (b) e o HDM, onde o MOSFET principal é substituído por um DM equivalente de mesma área de porta.

Figura 23 - Circuito elétrico equivalente de um MOSFET de geometria de porta Diamante Híbrido contendo três MOSFETs, sendo um de geometria hexagonal e outros dois com geometrias de porta retangular.

Figura 24 - Leiaute do MOSFET do tipo retangular feito no software ICStation.

Figura 25 - Leiaute do MOSFET do tipo Diamante Híbrido com ângulo $\alpha$ de $45^{\circ}$ feito no software ICStation.

Figura 26 - Leiaute do MOSFET do tipo Diamante Híbrido com ângulo $\alpha$ de $90^{\circ}$ feito no software ICStation.

Figura 27 - Leiaute do MOSFET do tipo Diamante Híbrido com ângulo $\alpha$ de $135^{\circ}$ feito no software ICStation.

Figura 28 - Curvas das correntes entre o dreno e a fonte em função da tensão entre a porta e a fonte dos HDMs e o CM, para uma $\mathrm{V}_{\mathrm{DS}}$ igual a $50 \mathrm{mV}$. 46

Figura 29 - Curvas das correntes entre o dreno e a fonte normalizada em função da razão de aspecto em função da sobretensão de porta dos HDMs com diferentes ângulos $\alpha$ e do CM, para uma VDS igual a $700 \mathrm{mV}$ (Região de saturação). 
Figura 30 - Curvas das resistências entre o dreno e a fonte normalizadas pela razão de aspecto dos HDMs e o CM em função de $\mathrm{V}_{\mathrm{GT}}$, para uma $\mathrm{V}_{\mathrm{DS}}$ igual a $50 \mathrm{mV}$.

Figura 31 - Curvas das transcondutâncias normalizadas pelas razões de aspecto dos HDMs e do CM em função da $V_{G T}$ (a) e em função de $\mathrm{IDS}_{\mathrm{DS}} /(\mathrm{W} / \mathrm{L})$ (b), considerando uma tensão entre o dreno e a fonte igual a $700 \mathrm{mV}$

Figura 32 - Gráfico de IDS/(W/L) em função de $V_{\text {GT }}$ na escala logarítmica dos MOSFETs com leiautes de porta dos tipos Diamante Híbrido e retangular, para uma $\mathrm{V}_{\mathrm{DS}}$ igual a $700 \mathrm{mV}$.....53 Figura 33 - Curvas experimentais das razões de $g_{m} / I_{D S}$ em função da corrente entre o dreno e a fonte normalizada em função da razão de aspecto [IDS/(W/L)] dos MOSFETs com leiautes de porta dos tipos Diamante Híbrido e retangular.

Figura 34 - Curvas experimentais das razões de $\mathrm{g}_{\mathrm{m}} / \mathrm{I} \mathrm{DS}$ em função da sobretensão de porta dos MOSFETs com leiautes de porta dos tipos Diamante Híbrido e retangular.

Figura 35 - Curvas experimentais das IDS/(W/L) em função da $V_{D S}$, considerando-se uma $V_{G T}$ igual $700 \mathrm{mV}$, dos MOSFETs com leiautes de porta dos tipos Diamante Híbrido e retangular.

Figura 36 - Curvas experimentais das condutâncias de saída normalizadas pela razão de aspecto $\left[\mathrm{g}_{\mathrm{D}} /(\mathrm{W} / \mathrm{L})\right]$ em função da tensão entre o dreno e a fonte dos MOSFETs com leiautes de porta dos tipos Diamante Híbrido e o retangular.

Figura 37 - Curvas de Av em função da VGT (a) e IDS/(W/L) (b) dos MOSFETs com leiautes de porta dos tipos Diamante Híbrido e retangular, para um $\mathrm{V}_{\mathrm{DS}}=700 \mathrm{mV}$.

Figura 38 - Tensão Early em função da sobretensão de porta experimental dos MOSFETs com leiautes de porta dos tipos Diamante Híbrido e o retangular, para uma $\mathrm{V}_{\mathrm{DS}}$ igual a $700 \mathrm{mV} . .63$ Figura 39 - Curvas da THD em função de $V_{\text {GT }}$ (a) e em função $g_{m} / I_{D S}$ (b) dos MOSFETs, considerando uma $\mathrm{V}_{\mathrm{DS}}$ igual a $700 \mathrm{mV}$, Va igual a $50 \mathrm{mV}$ e com $0 \mathrm{~V} \leq \mathrm{V}_{\mathrm{GT}} \leq 0,7 \mathrm{~V}$. Figura 40 - Curvas da dgm/(W/L)/dVGT em função de $V_{\text {GT }}$ dos HDMs e do CM, para uma VDS igual a $700 \mathrm{mV}$.

Figura 41 - THD/Av em função de $V_{\text {GT }}$ e em função de $\mathrm{g}_{\mathrm{m}} / \mathrm{IDS}_{\mathrm{DS}}$ dos MOSFETs dos tipos Diamante Híbrido com ângulos iguais a $45^{\circ}, 90^{\circ}$ e $135^{\circ}$ e do retangular para um Va igual a 50 $\mathrm{mV}$ (a) e uma $\mathrm{V}_{\mathrm{DS}}$ igual a $700 \mathrm{mV}$ (b).

Figura 42 - Curva experimental de THD/Av em função de Va dos MOSFETs dos tipos Diamante Híbrido com ângulos iguais a $45^{\circ}, 90^{\circ}$ e $135^{\circ}$ e do retangular operando com um $\mathrm{V}_{\mathrm{GT}}$ igual a $600 \mathrm{mV}$ e um $\mathrm{V}_{\mathrm{DS}}$ igual a $700 \mathrm{mV}$. 


\section{LISTA DE TABELAS}

Tabela 1 - Tabela com os valores das dimensões dos MOSFETs estudados, no qual o valor de b é igual a $180 \mathrm{~nm}$ para todos os MOSFETs dos tipos Diamantes.

Tabela 2 - Valores das tensões de limiar para os diferentes dispositivos, extraído com uma $\mathrm{V}_{\mathrm{DS}}$ igual a $50 \mathrm{mV}$ 46

Tabela 3 - Valores das transcondutâncias máximas dos MOSFETs com leiautes de porta dos tipos Diamante Híbrido e retangular para uma $\mathrm{V}_{\mathrm{DS}}$ igual a $700 \mathrm{mV}$.

Tabela 4 - Valores de SS, Ion, Ioff e Ion/Ioff dos MOSFETs com leiautes dos tipos Diamante Híbrido e retangular, considerando-se uma VDS igual a $700 \mathrm{mV}$

Tabela 5 - Valores dos ganhos de tensão em função de $\mathrm{V}_{\mathrm{GT}}$ dos HDMs $\left(\alpha=45^{\circ} ; 90^{\circ} ; 135^{\circ}\right) \mathrm{e}$ o CM equivalente, considerando-se uma $\mathrm{V}_{\mathrm{DS}}$ igual a $700 \mathrm{mV}$..... 60

Tabela 6 - Valores das tensões de Early em função da sobretensão de porta dos HDMs $(\alpha=$ $45^{\circ} ; 90^{\circ} ; 135^{\circ}$ ) e o $\mathrm{CM}$ equivalente, considerando-se uma $\mathrm{V}_{\mathrm{DS}}$ igual a $700 \mathrm{mV}$.

Tabela 7 - THD/Av em função da Va dos MOSFETs dos tipos Diamantes Híbridos e retangular para um valor de $\mathrm{V}_{\mathrm{DS}}$ igual a $700 \mathrm{mV}$ e operando com uma $\mathrm{V}_{\mathrm{GT}}$ igual a $600 \mathrm{mV} .70$ 


\section{ÍNDICE}

1 INTRODUÇÃO .............................................................................................................................. 11

2 CONCEITOS FUNDAMENTAIS _.................................................................................

2.1 TECNOLOGIA CONVENCIONAL DO PROCESSO DE FABRICAÇÃO DE CIS CMOS

2.2 TENSÃO DE LIMIAR DO MOSFET DO TIPO ENRIQUECIMENTO 17

2.3 TRANSCONDUTÂNCIA DO MOSFET 18

2.4 A RAZÃO gm/Ids EM FUNÇÃO DA Ids NORMALIZADO PELA RAZÃO DE ASPECTO (W/L) DOS MOSFETS

2.5 TENSÃO EARLY E CONDUTÂNCIA DE DRENO DO MOSFET. 20

2.6 GANHO DE TENSÃO DE UM AMPLIFICADOR DE TRANSCONDUTÂNCIA DE UM ÚNICO MOSFET .22

2.7 RESISTÊNCIA SÉRIE 23

2.8 DISTORÇÃO HARMÔNICA. 24

2.9 MÉTODOS PARA DETERMINAR A DISTORÇÃO HARMÔNICA EM MOSFETS ..26

2.10 GEOMETRIAS DE PORTA NÃO CONVENCIONAIS PARA O MOSFET . 33

2.11 MOSFET DO TIPO DIAMANTE HÍBRIDO 37

3 ESTUDO EXPERIMENTAL COMPARATIVO ENTRE MOSFETS DOS TIPOS DIAMANTE HÍBRIDO E CONVECIONAL

3.1 CARACTERÍSTICAS DIMENSIONAIS DOS MOSFETS UTILIZADOS PARA REALIZAR O ESTUDO EXPERIMENTAL 42

3.2 TENSÃO DE LIMIAR 45

3.3 CURVA [IDs/(W/L)] EM FUNÇÃO DE VGT 47

3.4 CURVA [RDS*(W/L)] EM FUNÇÃO DE VGT 48

3.5 TRANSCONDUTÂNCIA MÁXIMA DO MOSFET 50

3.6 INCLINAÇÕES DE SUBLIMIAR DOS MOSFETS COM LEIAUTES DE PORTA TIPO DIAMANTE HÍBRIDO E DO TIPO RETANGULAR 52 
3.7 CURVA DE gm/IDs EM FUNÇÃO DE Ids/(W/L) PARA OS MOSFETS COM LEIAUTES DE PORTA DOS TIPOS DIAMANTE HÍBRIDO E RETANGULAR 55

3.8 CURVA IDs/(W/L) EM FUNÇÃO DE VDs E CONDUTÂNCIA DE DRENO 57

3.9 VALORES DE VEA E Av DOS MOSFETS COM LEIAUTES DE PORTA DOS TIPOS DIAMANTE HÍBRIDO E DO TIPO RETANGULAR 59

3.10 DISTORÇÕES HARMÔNICAS PARA OS MOSFETS OPERANDO NA REGIÃO DE SATURAÇÃO 63

3.10.1 Distorção Harmônica Total (THD) 63

3.10.2 Distorção Harmônica Total normalizada pelo ganho de tensão (THD/Av). 66 3.10.3 Distorção Harmônica Total normalizada pelo ganho de tensão em função da amplitude do sinal de entrada senoidal (Va) 68

4 CONCLUSÃO

REFERÊNCIAS

APÊNDICE A - ARTIGOS PUBLICADOS ....................................................................... 79 


\section{INTRODUÇÃO}

$\mathrm{Na}$ atualidade, há uma demanda muito grande por sistemas eletrônicos em um único circuito integrado (Systems-on-a-Chip, SoCs) e essa demanda tem a tendência de crescer mais e mais porque eles são baratos, confiáveis e leves [1][2].

Para poder atender essa demanda, é necessário melhorar cada vez mais os parâmetros elétricos (Transcondutância, tensão Early, etc.) e as figuras de mérito (Ganho de tensão, Distorção Harmônica Total, etc.) do transistor de efeito de campo metal-óxido-semicondutor (Metal-Oxide-Semiconductor, MOS, Field Effect Transistor, MOSFET), medida que deve aumentar o seu fator de integração em circuitos integrados, isto é, diminuir as suas dimensões físicas $[1][2][3]$.

$\mathrm{Na}$ grande maioria dos SoCs, existem partes analógicas implementadas em conjunto com os blocos digitais, sensores etc., e uma figura de mérito muito importante para as aplicações analógicas é a distorção harmônica [1][2].

A distorção harmônica é a figura de mérito responsável por quantificar a linearidade de um MOSFET [4][5]. A não linearidade no sinal, isto é, a distorção harmônica presente no sinal, é prejudicial em aplicações que necessitam de uma alta fidelidade do sinal, como por exemplo em aplicações médicas. Por isso, há a necessidade de MOSFETs que possuam uma baixa distorção harmônica [4].

Visando a redução da distorção harmônica, diferentes frentes de pesquisa estão sendo utilizadas para poder atingir esse objetivo, como por exemplo, o uso de dispositivos silício sobre isolante (Silicon-on-Insulator, SOI) de porta circundante (Gate-all-around, GAA), com a presença da estrutura de canal gradual (Graded-Channel, GC) e também sem ela, em que o GC GAA apresentou uma distorção harmônica total (THD) $30 \mathrm{~dB}$ menor, considerando as mesmas condições de polarização, na saturação, em relação à do GAA convencional, pois o GC GAA apresentou um ganho de tensão intrínsecos (Av) cerca de 50 vezes maior que os Avs obtidos nos transistores GAA convencionais [6].

Também há na literatura a pesquisa de transistores de nano fios sem junção (Junctionless Nanowire Transistors, JNT) com a temperatura variando entre $223 \mathrm{~K}$ e $473 \mathrm{~K}$, visando a redução da distorção harmônica. O resultado desse trabalho mostrou que a THD desse dispositivo é reduzida à medida que a temperatura aumentava, em relação ao trigate no modo inversão (Inversion Mode, IM), considerando as mesmas condições de polarização [em que apresentou uma THD cerca de 1,5 dB menor para um valor da razão entre a transcondutância e a corrente entre o dreno e a fonte $\left(\mathrm{g}_{\mathrm{m}} / \mathrm{IDS}\right)$ igual a $10 \mathrm{~V}^{-1}$ ], isso ocorreu em função do aumento 
do Av desses dispositivos com a elevação da temperatura [7]. Enquanto a temperatura era reduzida, a THD do JNT aumentava [7].

E há, também, a utilização dos MOSFETs de porta anelar circular, em que esses dispositivos com a configuração de dreno externo foram responsáveis por gerarem uma menor distorção harmônica total normalizada pelo ganho de tensão (THD/Av), cerca de 14,9 dB, para uma sobretensão de porta igual a 0,47 V e com uma tensão da amplitude do sinal de entrada de $30 \mathrm{mV}$ em relação ao SOI MOSFET de porta retangular. Isso foi justificado devido ao maior ganho de tensão do MOSFET de porta anelar circular operando com a configuração de dreno externo, resultando dessa forma uma menor (THD/Av) [8]

Mais recentemente foram propostos inovadores estilos de leiaute de porta para o MOSFET, que estão baseados na "Engenharia de Junção PN entre as Regiões de Dreno/Fonte e Canal" [9][10], ou seja, o uso de geometrias não convencionais (diferentes das retangulares que são utilizadas atualmente) para a região de porta dos MOSFETs para poder melhorar o desempenho elétrico dos MOSFETs, e consequentemente a dos CIs CMOS analógicos graças aos efeitos de canto longitudinal (Longitudinal Corner Effect, LCE) e o efeito da associação paralela de MOSFETs com diferentes comprimentos de canal (Parallel conection of MOSFETS with Different Channel Lengths Effect, PAMDLE) presentes em sua estrutura [9][11], sem gerar qualquer custo adicional para os atuais e sofisticados processos de fabricação planares de CIs CMOS [9].

Os estilos de leiaute de porta, para os MOSFETs dos tipos hexagonal (Diamante) [9] (Figura 1), octogonal (OCTO) (Figura 2) [12], e elipsoidal (Figura 3) para MOSFETs [13] são exemplos dessas geometrias de porta.

Porém, devido às regras de projeto mais recentes de algumas tecnologias de fabricação de CIs CMOS, não é permitido que a região de porta não seja ortogonal à região ativa do MOSFET. Dentro desse contexto, para poder sanar esse problema, foi proposto o MOSFET do tipo Diamante Híbrido (Hybrid Diamond MOSFET, HDM), em que a sua região de porta é composta de dois MOSFETs de porta retangular idênticos ligados em paralelo ao MOSFET do tipo Diamante, conforme ilustrado pelo leiaute da Figura 4. 
Figura 1 - Fotografia de um MOSFET do tipo Diamante, do tipo n, implementado com tecnologia convencional (Bulk) de $350 \mathrm{~nm}$ da ON-Semiconductor.

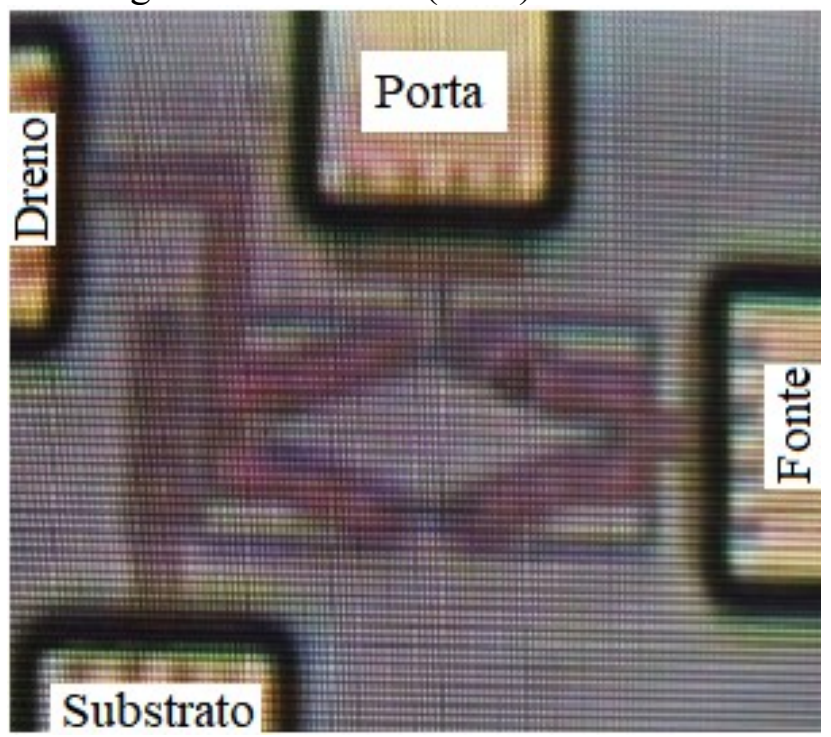

Fonte: Autor "Adaptado de" Gimenez, 2015

Figura 2 - Fotografia de um MOSFET do tipo Octagonal, do tipo n, implementado com tecnologia SOI de $1 \mu \mathrm{m}$ da Universidade Católica de Louvain La Nueve (UCL), da Bélgica.

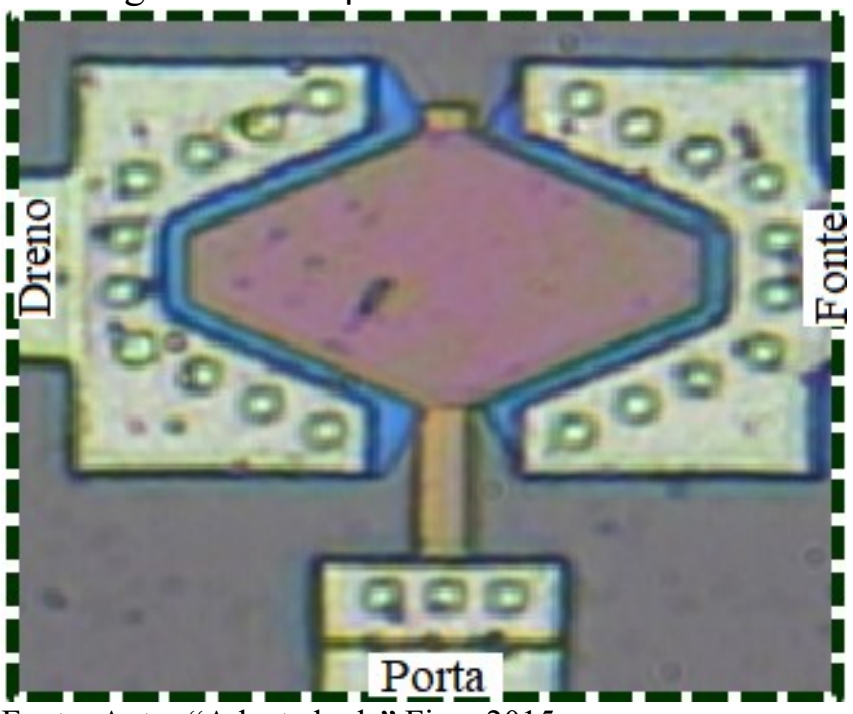

Fonte: Autor "Adaptado de" Fino, 2015 
Figura 3 - Fotografia de um MOSFET do tipo Elipsoidal, do tipo n, implementado com tecnologia Bulk de $350 \mathrm{~nm}$ da ON-Semiconductor.

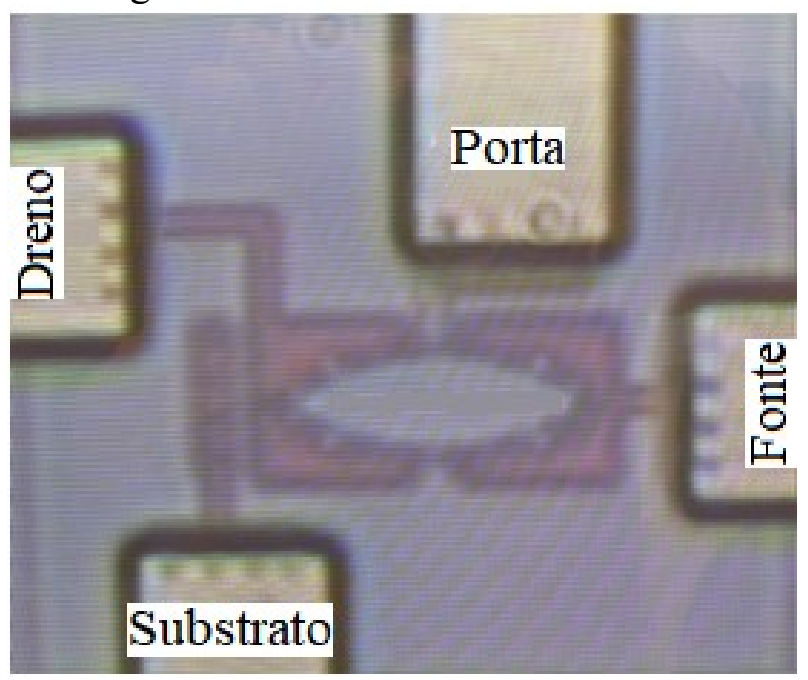

Fonte: Fonte: Autor “Adaptado de" Gimenez, 2015

Figura 4 - Leiaute simplificado de um MOSFET do tipo Diamante-Híbrido, do tipo n, de tecnologia Bulk.

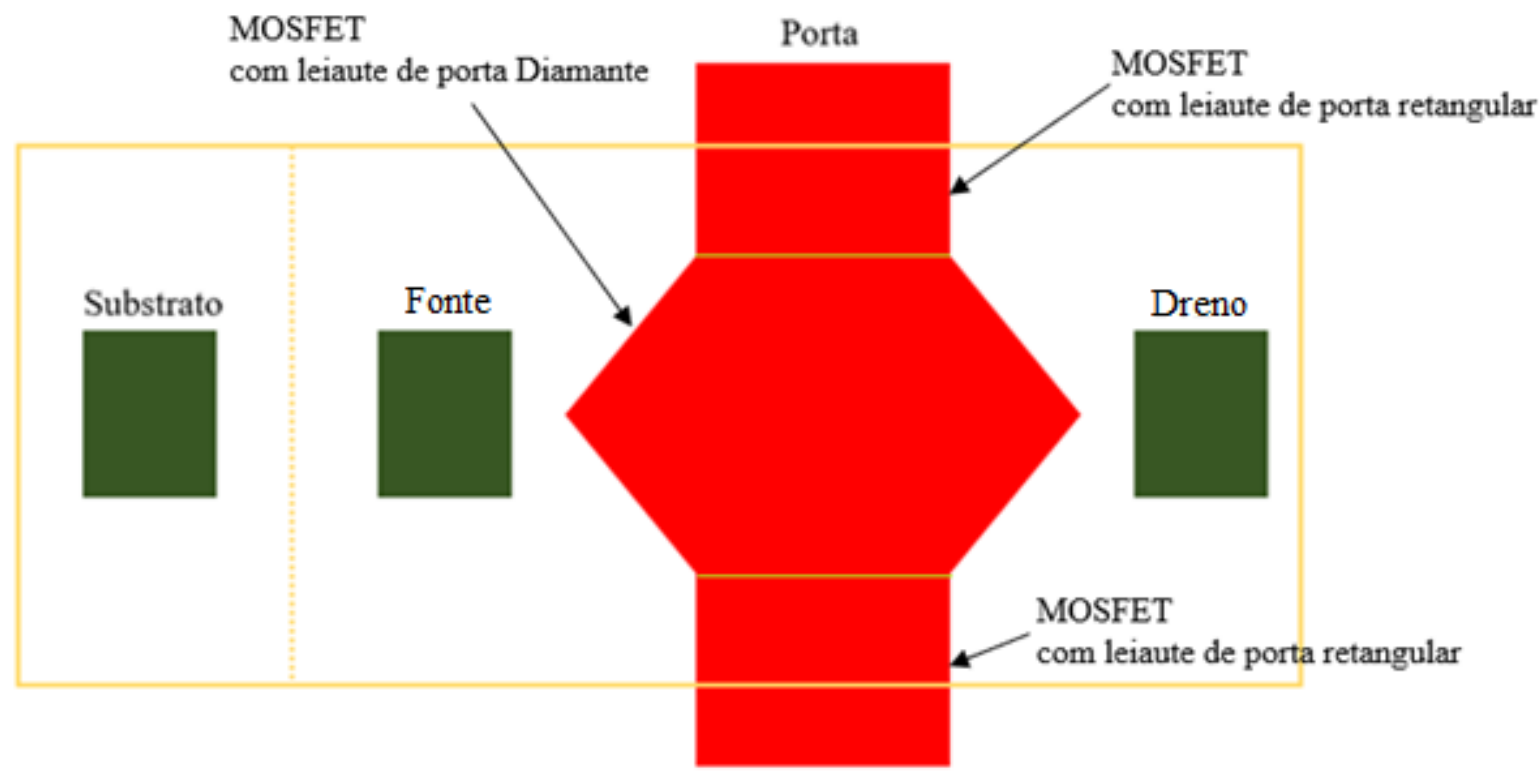

Fonte: Fonte: Autor

Dentro deste contexto, e sabendo que os HDMs nunca tiveram os seus desempenhos elétricos explorados pela literatura, a motivação desse projeto de mestrado é de estudar as principais figuras de mérito e parâmetros elétricos dos HDMs em relação aos MOSFETs de porta retangular convencional (CM), em especial nas aplicações analógicas, onde, geralmente, os dispositivos apresentam dimensões físicas elevadas.

Sendo assim, o objetivo principal desse trabalho é comparar experimentalmente como ocorre o efeito da distorção harmônica total normalizada pelo ganho de tensão no MOSFET do tipo Diamante Híbrido em relação ao CM de 180 nm da TSMC. 


\section{CONCEITOS FUNDAMENTAIS}

Este capítulo aborda os principais conceitos teóricos que são necessários para o entendimento dessa dissertação. Nele são discutidos assuntos pertinentes à tecnologia convencional de fabricação de CIs CMOS, aos MOSFETs, à tensão de limiar (VTH), à transcondutância $\left(\mathrm{g}_{\mathrm{m}}\right)$, à razão $\mathrm{g}_{\mathrm{m}} / \mathrm{I}_{\mathrm{DS}}$, à tensão Early $\left(\mathrm{V}_{\mathrm{EA}}\right)$, à resistência série $\left(\mathrm{R}_{\mathrm{s}}\right)$ do MOSFET, ao ganho de tensão intrínseco, à distorção harmônica (linearidade), aos métodos para determinação da distorção harmônica e às geometrias não convencionais de porta para os MOSFETs.

\subsection{TECNOLOGIA CONVENCIONAL DO PROCESSO DE FABRICAÇÃO DE CIs CMOS}

O MOSFET é um dispositivo eletrônico capaz de controlar o fluxo da corrente elétrica entre os seus terminais de dreno (D) e fonte (S) através de um potencial aplicado entre os seus terminais de porta $(\mathrm{G})$ e fonte e entre o dreno e a fonte [1][14].

$\mathrm{Na}$ tecnologia de fabricação de CIs CMOS convencionais, os MOSFETs são fabricados em lâminas de silício, com centenas de micrometros de espessura, mas, efetivamente, só a superfície da lâmina é utilizada para a fabricação desses dispositivos [1]. Um exemplo de uma secção transversal de um MOSFET do tipo n (nMOSFET) que foi fabricado utilizando o processo de fabricação convencional de CIs CMOS é ilustrado na Figura 5 [14].

Figura 5 - Secção transversal da estrutura de um MOSFET do tipo n construído com tecnologia Bulk CMOS de CIs.

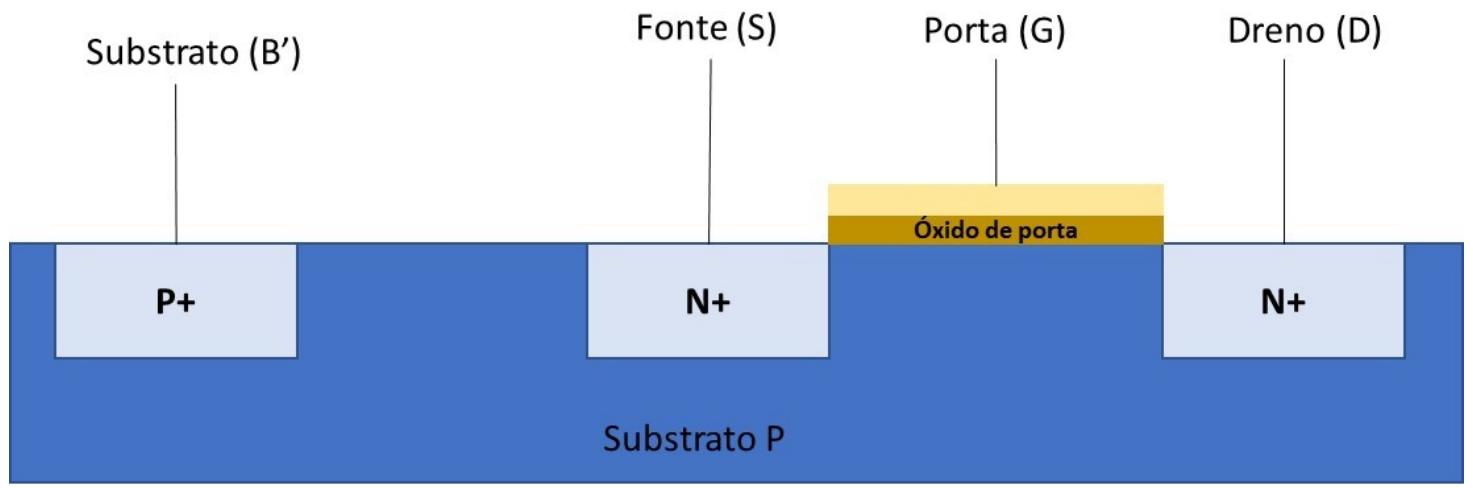

Fonte: Autor

Na Figura 5, o B' representa o substrato, N+ representa a região dopada do tipo N, P+ representa a região dopada do tipo $\mathrm{P}$ e o substrato $\mathrm{P}$. 
O MOSFET possui diversas aplicações, como por exemplo: um amplificador de sinais elétricos, chave eletrônica, sendo que sua função é interromper ou liberar a passagem de corrente elétrica para uma determinada carga [1], entre outras aplicações.

Para exemplificar, os MOSFETs planares, do tipo enriquecimento, podem ser classificados através do tipo de portador de carga predominante (elétron ou lacuna) em seu canal (Figura 6) [14][16]:

a) nMOSFET do tipo enriquecimento;

b) MOSFET do tipo $\mathrm{p}$ (pMOSFET) do tipo enriquecimento;

Figura 6 - Simbologias e as secções transversais dos MOSFETs.

a)

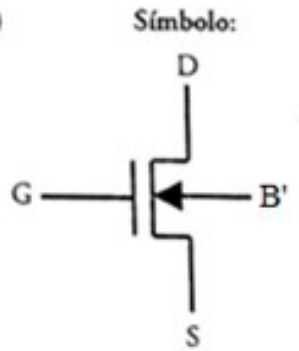

c)

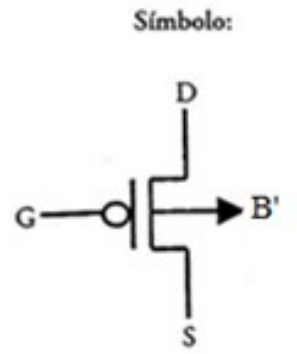

b)

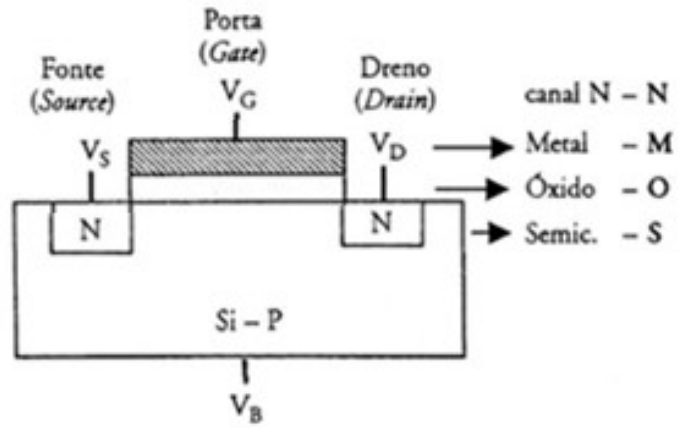

d)

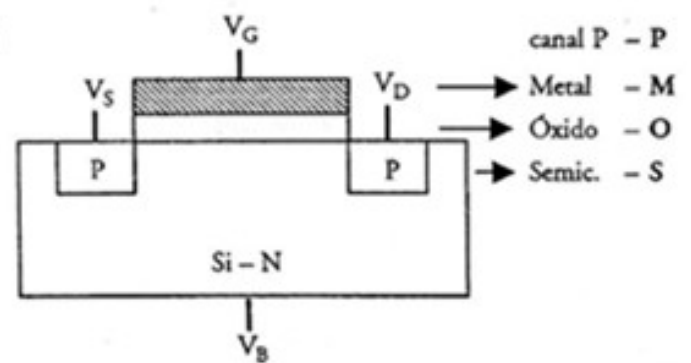

Fonte: Autor “Adaptado de” Martino, 2003, p.76

Na Figura 6, a) representa a simbologia de um nMOSFET do tipo enriquecimento; b) é a secção transversal de um nMOSFET do tipo enriquecimento; c) representa a simbologia de um pMOSFET do tipo enriquecimento; d) é a secção transversal de um nMOSFET do tipo enriquecimento; $V_{G}$ é a tensão de porta; $V_{D}$ representa a tensão de dreno; $V_{S}$ é a tensão de fonte; Vв é a tensão de substrato; $\mathrm{Si}-\mathrm{P}$ representa o silício dopado com dopantes do tipo p e $\mathrm{Si}-\mathrm{N}$ é o silício dopado com dopantes do tipo n.

O princípio de funcionamento do nMOSFET do tipo enriquecimento é baseado no controle das suas cargas no substrato posicionadas logo abaixo do óxido de porta [1][14].

O funcionamento do nMOSFET do tipo enriquecimento pode ser melhor detalhado da seguinte maneira: quando aplicada uma diferença de potencial entre os terminais de dreno e fonte $\left(V_{D S}\right)$ e com uma diferença de potencial entre a porta e a fonte $\left(V_{G S}\right)$ menor do que a 
tensão de faixa plana $\left(\mathrm{V}_{\mathrm{FB}}\right)$, praticamente não há passagem de corrente elétrica no transistor entre os seus terminais de fonte e dreno [16].

Contudo, se $\mathrm{V}_{\mathrm{GS}}$ for maior que $\mathrm{V}_{\mathrm{FB}}$, então este induzirá na superfície do silício do tipo P cargas negativas, ou seja, a criação da região de depleção antes da inversão de canal (Atração de cargas negativas) [1]. No início, terá a formação de uma região de depleção no substrato e à medida que $\mathrm{V}_{\mathrm{GS}}$ aumentar, maior será a região de depleção e, assim, maior será o campo elétrico vertical nessa região. Ao continuar aumentando $\mathrm{V}_{\mathrm{GS}}$, até atingir sua tensão de limiar, ocorre a formação de uma camada superficial de elétrons livres que possibilitará a condução da corrente elétrica entre o dreno e a fonte (IDS) [14]. Ao aumentar o $\mathrm{V}_{\mathrm{GS}}$ acima de $\mathrm{V}_{\mathrm{TH}}$, o transistor passa a operar no regime de inversão e terá um canal formado entre a região de fonte e dreno [15][16].

De maneira análoga, para o pMOSFET deve-se aplicar uma tensão negativa entre a porta e o substrato de tal forma que induza a formação de uma camada de lacunas, que permitirá a passagem de corrente IsD nesse dispositivo [1][16].

\subsection{TENSÃO DE LIMIAR DO MOSFET DO TIPO ENRIQUECIMENTO}

A tensão de limiar de um MOSFET do tipo enriquecimento, fabricado com tecnologia convencional de CIs CMOS, é definida como a tensão de porta capaz de criar uma camada de inversão na superfície do canal, isto é, na superfície abaixo da interface entre substrato do MOSFET e o óxido de porta, povoando-a com portadores minoritários, que são capazes de conduzir corrente elétrica ao longo do canal do MOSFET [3][17][18][19]. A tensão de limiar de um MOSFET convencional do tipo n é dado pela expressão mostrada na Equação (1) [3].

$$
V_{T H}=-\frac{q * N_{A} * d_{M A ́ X}}{C_{O X}}+2 * \varphi_{F}+V_{F B}
$$

Sendo q a carga elementar do elétron, $\mathrm{N}_{\mathrm{A}}$ a concentração por unidade de volume de portadores majoritários no substrato, dMÁx a espessura da região de depleção máxima, Cox a capacitância do óxido por unidade de área, $\varphi$ F o potencial de Fermi e VFB a tensão de faixa plana do Si [19].

Existem diversos métodos para extração de $\mathrm{V}_{\mathrm{TH}}$, como por exemplo, a extrapolação da região linear da corrente na curva da corrente entre o dreno e a fonte em função de $V_{G S}$ para VDS próximo de zero [18][19]. 
Neste trabalho foi usado o método da segunda derivada da corrente de dreno e fonte em função da $V_{G S}$, para $V_{D S}$ tendendo a zero, que também pode ser chamado de método da transcondutância [3][18][19], como é ilustrado na Figura 7.

Figura 7 - Gráfico da derivada da transcondutância de um nMOSFET em função da tensão de porta, para um $V_{D S}$ tendendo a zero (Neste caso $V_{D S}$ igual a $50 \mathrm{mV}$ ) utilizado para obter o valor de $\mathrm{V}_{\mathrm{TH}}$.

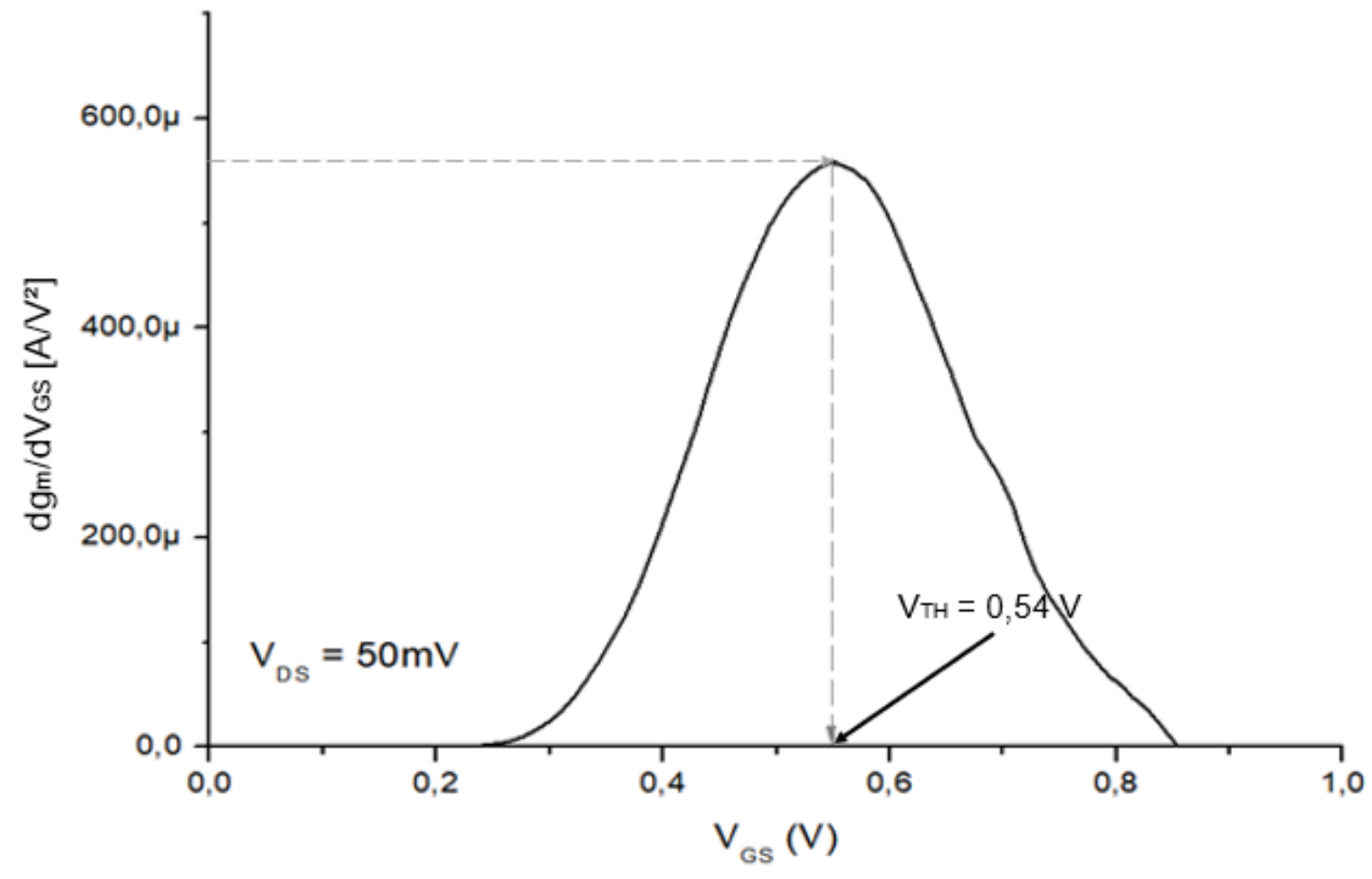

Fonte: Autor

\subsection{TRANSCONDUTÂNCIA DO MOSFET}

A transcondutância do MOSFET é a medida da efetividade do controle da corrente elétrica entre o dreno e a fonte pela tensão aplicada entre a porta e a fonte e é expressa através da Equação (2) [1][3].

$$
g_{m}=\frac{\Delta I_{D S}}{\Delta V_{G S}}=\frac{\partial I_{D S}}{\partial V_{G S}}
$$

Sendo $\Delta \mathrm{IDS}$ a variação da corrente elétrica entre fonte e dreno, $\Delta \mathrm{V}_{\mathrm{GS}}$ a variação de potencial entre porta e fonte, e $\partial \mathrm{IDS}_{\mathrm{DS}} \partial \mathrm{V}_{\mathrm{GS}}$ a derivada da corrente de dreno e fonte em relação às variações da tensão entre a porta e a fonte. 
Como descrito na Equação (2), a transcondutância é obtida através da primeira derivada da curva IDS em função de $V_{G S}$ do MOSFET, tanto na região de saturação, em que é utilizado como amplificador nas aplicações de CIs CMOS analógicos [3], quanto na região de triodo.

A Figura 8 ilustra de maneira gráfica a Equação (2), ou seja, a transcondutância do MOSFET em função da tensão entre porta e fonte. No ponto de máximo dessa curva se obtém o valor máximo da transcondutância do dispositivo $\left(\mathrm{g}_{\mathrm{m}} \mathrm{MAx}\right)$ [3].

Figura 8 - Gráfico da transcondutância em função das variações da tensão entre a porta e a fonte para poder obter a transcondutância máxima.

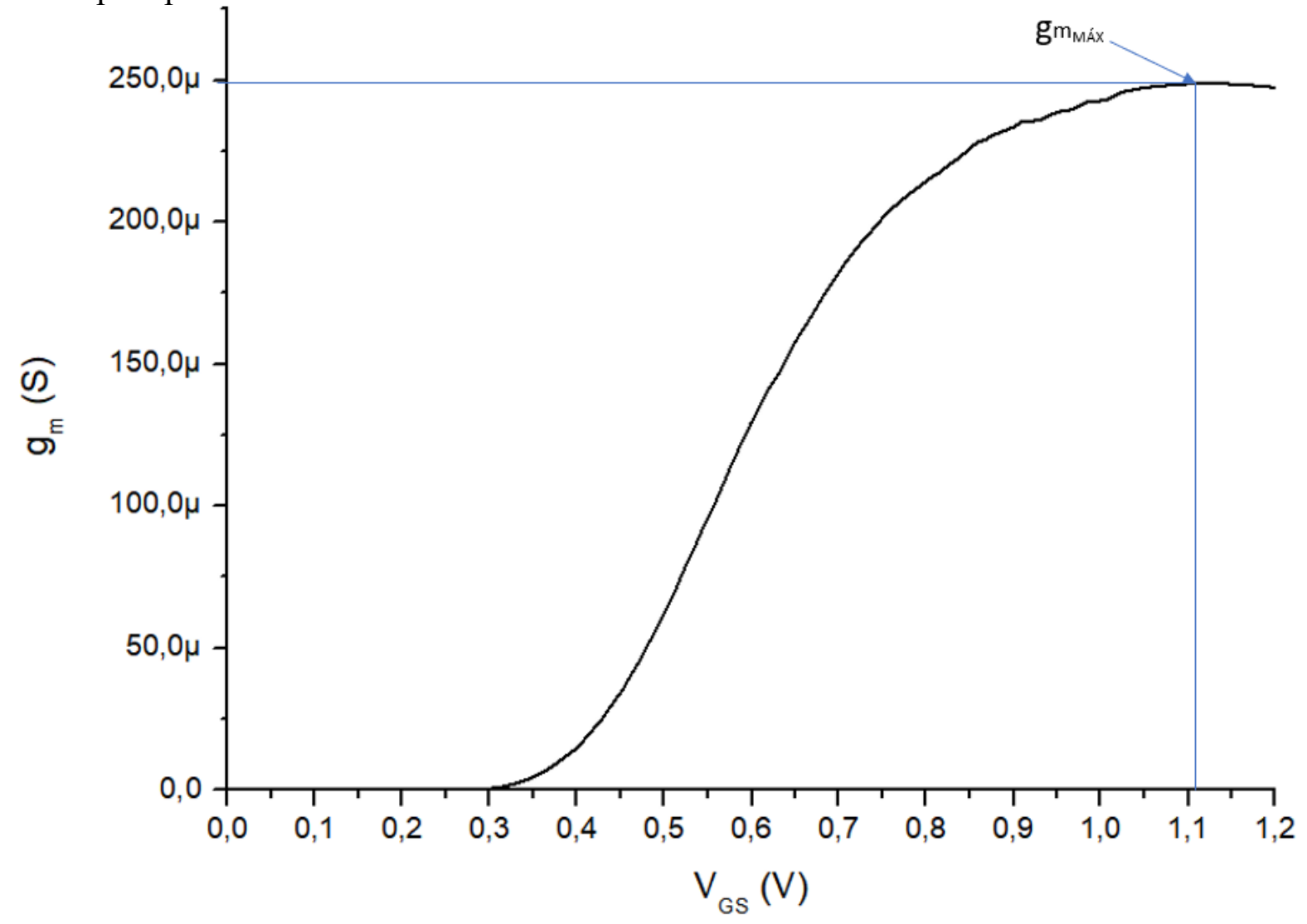

Fonte: Autor

\subsection{A RAZÃO gm/IDs EM FUNÇÃO DA IDs NORMALIZADO PELA RAZÃO DE ASPECTO (W/L) DOS MOSFETS}

A razão entre a transcondutância e a corrente entre o dreno e a fonte é um indicador do fator de qualidade do transistor no que diz respeito às suas características de amplificação de sinais [3][20]. Ela também é capaz de indicar em qual regime de inversão do canal o transistor está operando. É uma característica universal para todos os MOSFETs fabricados numa mesma tecnologia de CI CMOS e para um mesmo lote de fabricação [20]. 
Esse gráfico é utilizado para o projeto de circuitos analógicos CMOS de baixa tensão e baixa potência (Low Power - Low Voltage), em que a região de inversão de canal fraco possui um alto ganho de tensão, mas uma baixa velocidade de operação (resposta em frequências ou valores de frequência de ganho de tensão unitário, fT). Na região de inversão de canal moderado, é geralmente usada para implementar circuitos analógicos com um bom compromisso entre o ganho de tensão e velocidade de operação e a região de inversão de canal forte possui um baixo ganho de tensão, mas uma alta velocidade de operação [20].

A Figura 9 apresenta um exemplo de uma curva teórica da $g_{m} / I_{D S}$ em função da corrente de dreno e fonte normalizada pela razão de aspecto do MOSFET [IDS/(W/L)] [20].

Figura 9 - Exemplo de uma curva de $g_{m} / I_{D s}$ em função de IDs/(W/L) de um nMOSFET.

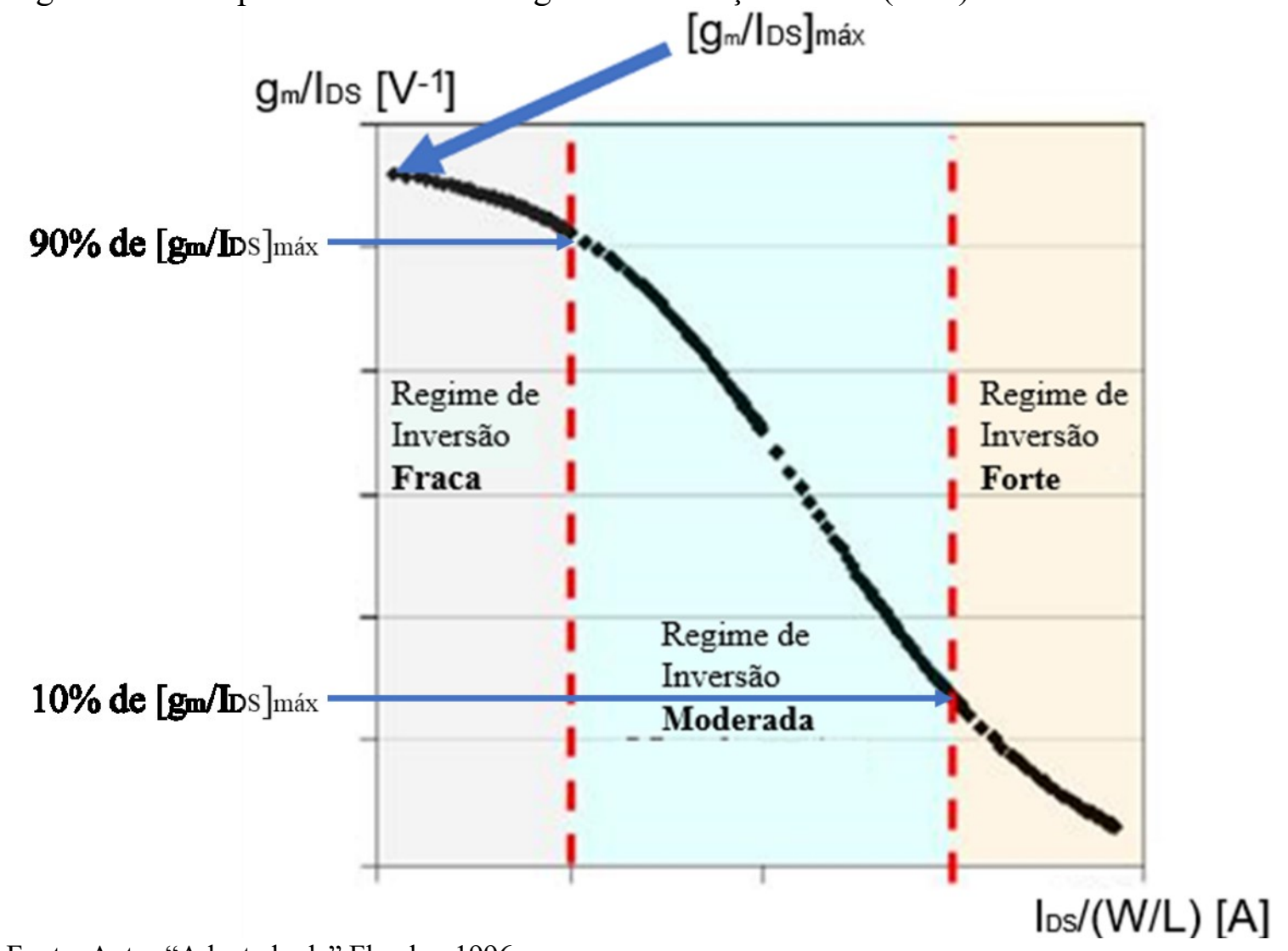

Fonte: Autor “Adaptado de" Flandre, 1996

\subsection{TENSÃO EARLY E CONDUTÂNCIA DE DRENO DO MOSFET}

A tensão Early é o parâmetro que mede a dependência da corrente elétrica entre o dreno e a fonte de saturação (IDSsat) em relação à variação da tensão entre dreno e a fonte, para uma determinada sobretensão de porta $\left(\mathrm{V}_{\mathrm{GT}}=\mathrm{V}_{\mathrm{GS}}-\mathrm{V}_{\mathrm{DS}}\right)$ ou para uma determinada $\mathrm{V}_{\mathrm{GS}}$ [1]. 
A tensão Early pode ser obtida, comumente, por meio da extrapolação da parte linear da região de saturação da curva IDS em função de VDS para um determinado valor da VGT , ou $\mathrm{V}_{\mathrm{GS}}$, até atingir o ponto em que a IDS é igual a 0 A [1][16], como ilustrado na Figura 10. O valor da tensão Early depende do comprimento de canal e da concentração de dopantes na região de canal [1], e é expresso pela Equação (3).

$$
V_{E A}=\frac{1}{\lambda}
$$

Sendo $\lambda$ o parâmetro que considera o efeito da modulação do comprimento de canal devido à tensão aplicada entre o dreno e a fonte no MOSFET [1].

Figura 10 - Ilustração esquemática da obtenção da $V_{E A}$ por meio da extrapolação da região linear da região de saturação da curva IDS em função de $V_{D S}$ para vários valores de $V_{G S}$ até atingir o ponto em que IDs é igual a $0 \mathrm{~A}$.

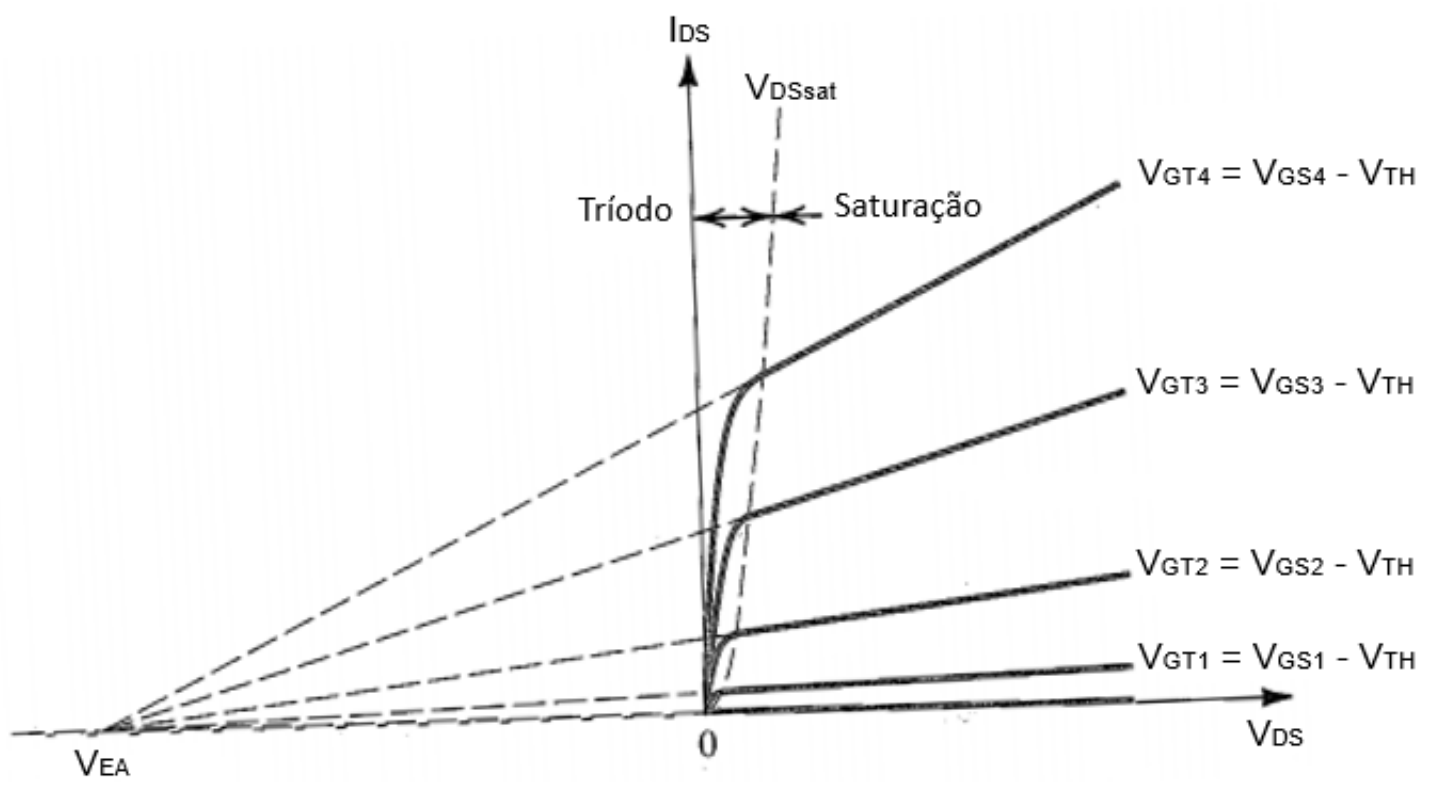

Fonte: Autor "Adaptado" de Colinge, 2002

Sendo $V_{G S 4}, V_{G S 3}, V_{G S 2}$ e $V_{G S 1}\left(V_{G S 4}>V_{G S 3}>V_{G S}>V_{G S 1}\right)$ as diferentes diferenças de potencial aplicadas entre a porta e a fonte, $\mathrm{V}_{\mathrm{GT} 4}, \mathrm{~V}_{\mathrm{GT}}, \mathrm{V}_{\mathrm{GT} 2}$ e $\mathrm{V}_{\mathrm{GT} 1}$ as sobretensões de porta e o $V_{D S s a t}\left(V_{D S s a t} \geq V_{G S}-V_{T H}\right)$ a tensão mínima aplicada entre os terminais de fonte e dreno para que o MOSFET opere na região de saturação.

Um outro método para a obtenção da VEA é por meio da razão de IDs e a condutância de dreno dos MOSFETs, em que é expresso matematicamente por intermédio da Equação (4). Este método foi utilizado para a obtenção da VEA dos MOSFETs usados neste projeto de pesquisa. 


$$
V_{E A}=\frac{I_{D S}}{g_{D}}
$$

Sendo $g_{D}$ a condutância de dreno, também conhecida como condutância de saída do MOSFET.

A condutância de dreno é obtida através da variação da corrente de dreno e fonte em função da variação da tensão entre o dreno e a fonte, para uma determinada tensão entre a porta e a fonte, de acordo com a Equação (5) [3][21].

$$
g_{D}=\left.\frac{\partial I_{D S}}{\partial V_{D S}}\right|_{V_{G S}=\text { constante }}
$$

\subsection{GANHO DE TENSÃO DE UM AMPLIFICADOR DE TRANSCONDUTÂNCIA DE UM ÚNICO MOSFET}

O ganho de tensão intrínseco do MOSFET é uma figura de mérito que descreve o quanto ele pode aumentar a amplitude de um sinal aplicado à sua entrada em sua saída [1][3].

A obtenção do Av do MOSFET é representada pela Figura 11, em que se encontra ilustrado um circuito elétrico simples de um MOSFET ligado a uma carga capacitiva $\mathrm{C}_{\mathrm{L}}$ na saída do MOSFET, sendo que o VDD é a tensão de alimentação, VIN é a tensão de entrada e Vout é a tensão de saída [8].

Figura 11 - Circuito amplificador de transcondutância de um único MOSFET.

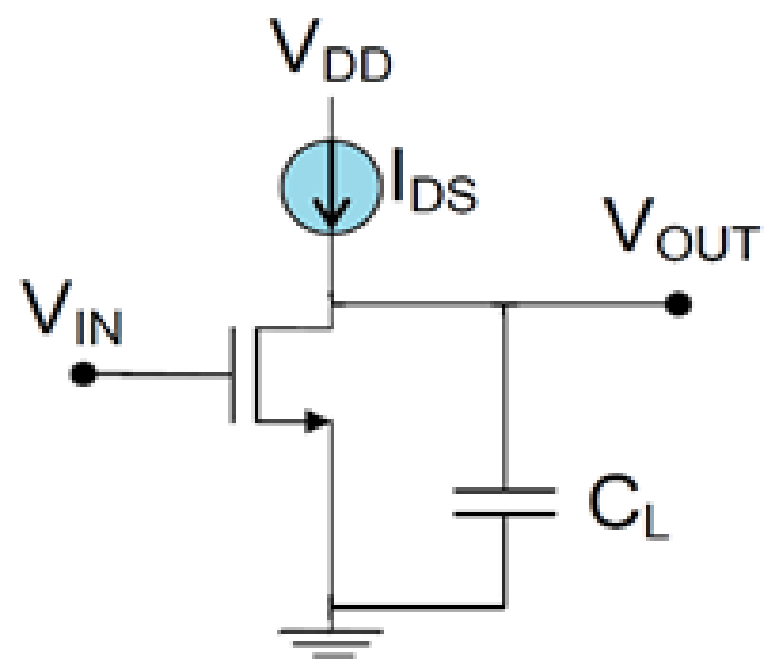

Fonte: Autor “Adaptado" de Dantas, 2008 
Analisando a Figura 11, é possível obter o valor de Av desse circuito elétrico, através da Equação (6) [3].

$$
A_{V}=\frac{\Delta V_{O U T}}{\Delta V_{I N}}=\frac{\Delta V_{D S}}{\Delta V_{G S}}=\frac{g_{m}}{g_{D}} \cong \frac{g_{m}}{I_{D S}} * V_{E A}
$$

Sendo $\Delta$ V OUT a variação da tensão de saída, $\Delta V_{\text {IN }}$ a variação da tensão de entrada, $\Delta V_{D S}$ a variação do potencial elétrico entre o dreno e a fonte [3].

Ao examinar a Equação (6), nota-se que para maximizar o Av é necessário que os MOSFETs tenham maiores valores de $g_{m}$ e menores valores de $g_{D}[1]$.

Os maiores valores de $\mathrm{g}_{\mathrm{m}} / \mathrm{IDS}$ ocorrem no regime de inversão fraca do MOSFET, visto que $\mathrm{g}_{\mathrm{m}} /$ IDs é praticamente constante e é inversamente proporcional a inclinação de sublimiar (SS) o que induz em um maior valor do Av [3]. Já os maiores valores de VEA são encontrados quando se utilizam MOSFETs com comprimentos de canal longos que são menos susceptíveis aos efeitos de canal curto [1].

\subsection{RESISTÊNCIA SÉRIE}

A resistência série $\left(\mathrm{R}_{\mathrm{s}}\right)$ de um MOSFET é o valor da resistência intrínseca das regiões de dreno e fonte do dispositivo, que interfere diretamente no valor da corrente de dreno [1].

Considerando a curva IDS em função de $V_{G S}$ do MOSFET para um valor de VDS constante, e dividindo VDS por IDS, determina-se a resistência total entre o dreno e a fonte do dispositivo (RDS), Equação (7) [3].

$$
R_{D S}=\frac{V_{D S}}{I_{D S}}=R_{C H}+R_{S}
$$

sendo que na Equação (7) o $\mathrm{R}_{\mathrm{CH}}$ é a resistência do canal do MOSFET.

Para baixos valores de $V_{D S}$ e altos valores de $V_{G S}$, o valor de $R_{C H}$ cai drasticamente, pois a interface entre o canal e o óxido de porta apresenta-se totalmente invertida, fazendo com que RSD se torne praticamente igual a Rs [3]. 


\subsection{DISTORÇÃO HARMÔNICA}

A distorção harmônica, ou a não-linearidade de um sistema, é qualquer deformação na forma de onda de um sinal de saída em relação à forma de onda do sinal de entrada, seja ele amplificado ou não [22][23][24][25].

O sinal da saída de um sistema pode sofrer dois tipos distintos de distorções, podendo estas serem lineares ou não-lineares [23].

- Distorção linear: A distorção é dita linear quando um sinal de entrada senoidal de frequência f e amplitude A entra no sistema e o sinal de saída também é senoidal e de frequência f. No entanto, as amplitudes e fases podem ser diferentes. Um exemplo típico desses sistemas são os filtros. Neste caso, não há formação de novos harmônicos no sinal de saída, apenas ocorre a supressão de algumas faixas de frequência [22][23].

- Distorção não-linear: A distorção é dita não-linear quando num dado sistema apresentar na sua entrada um sinal senoidal com frequência fundamental f e amplitude (AIN), e, em sua saída, o sinal tenha uma amplitude diferente da entrada (Aout) e com um espectro de frequências composto por múltiplos inteiros da frequência fundamental: $2 \mathrm{f}, 3 \mathrm{f}, \ldots$. É por essa razão que a distorção não-linear também pode ser denominada distorção harmônica [23]. Um exemplo de distorção não-linear ocorre em circuitos com diodos retificadores e em amplificadores [23].

Ambas as distorções, tanto a linear quanto a não-linear, são ilustradas na Figura 12 [8], através do espectro de frequências. 
Figura 12 - Efeito da distorção linear (a) e não-linear (b) sobre o espectro de f de um sistema eletrônico.

Sinal de Entrada

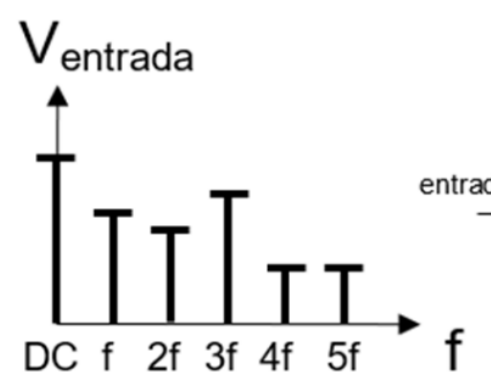

\section{Sinal de Saída}

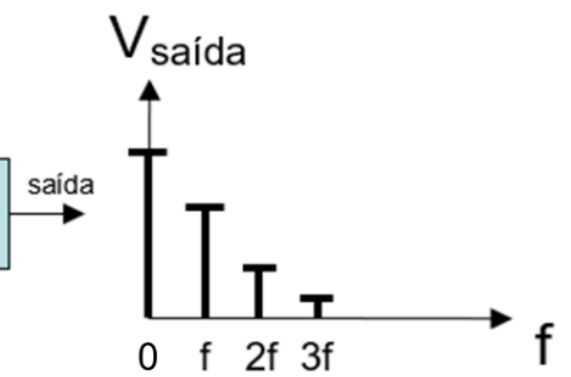

(a)

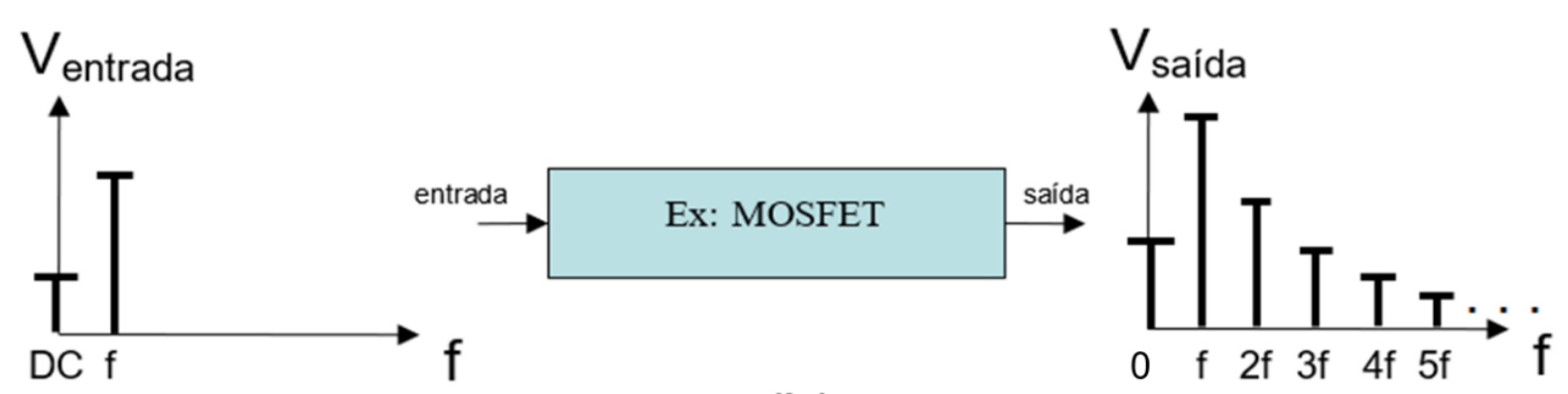

(b)

Fonte: Autor “Adaptado” de Dantas, 2008

$\mathrm{Na}$ Figura 12, $\mathrm{V}_{\text {entrada }}$ corresponde ao sinal de entrada quando aplicado ao sistema eletrônico, $\mathrm{V}_{\text {saída }}$ é o sinal de saída dado pelo sistema eletrônico, DC corresponde a componente contínua do sinal, f é a frequência fundamental, $2 \mathrm{f}$ é a segunda harmônica, $3 \mathrm{f}$ é a terceira harmônica, 4f é a quarta harmônica e, por fim, 5 f é a quinta harmônica [8].

A medida da distorção harmônica no MOSFET é medida aplicando-se um sinal variável entre os terminais da porta e da fonte $\left[\mathrm{V}_{\mathrm{GS}}=\mathrm{V}_{\mathrm{o}}+\mathrm{Va} \cdot \operatorname{sen}\left(2 . \pi\right.\right.$.f.t), sendo $\mathrm{V}_{\mathrm{o}}$ o nível de tensão de corrente contínua entre os terminais da porta e da fonte usado com a tensão de operação do sistema, Va a tensão da amplitude máxima do sinal senoidal de entrada, $\mathrm{f}$ a frequência do sinal senoidal de entrada e t o período] e, posteriormente, se deve medir a IDs para uma determinada VDS [23].

A Figura 13.(a) apresenta o circuito elétrico que foi utilizado para realizar a medida da distorção harmônica dos MOSFETs utilizados neste projeto de pesquisa e a Figura 13.(b) representa, de maneira ilustrada, a distorção harmônica do sinal de saída em decorrência da não-linearidade da curva característica de IDS em função da $V_{G S}$, para um determinado valor de VDS, em relação ao sinal aplicado em sua entrada [8]. 
Figura 13 - Circuito utilizado para realizar a medida da distorção harmônica num nMOSFET (a) e a distorção da corrente de dreno devido a não-linearidade na curva IDs em função da $\mathrm{V}_{\mathrm{GS}}$, para um determinado valor de $\mathrm{V}_{\mathrm{DS}}$ (b) quando é amplificado um sinal senoidal de amplitude $\mathrm{Va}$ e frequência f na entrada do MOSFET $\left(\mathrm{V}_{\mathrm{GS}}=\mathrm{Vo}_{\mathrm{o}}+\mathrm{Va}^{*} \operatorname{sen}(\omega . \mathrm{t})\right)$.

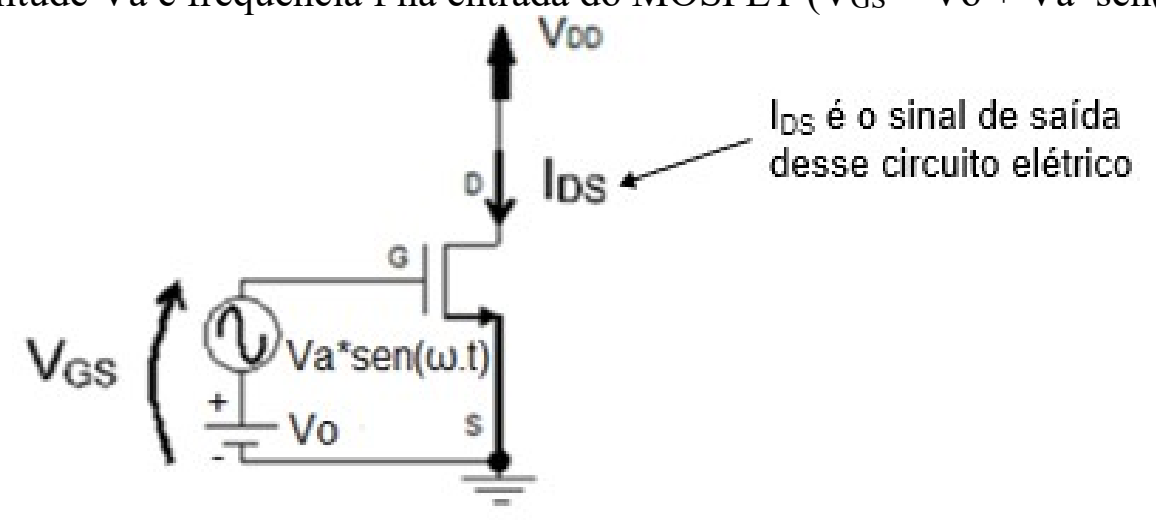

(a)

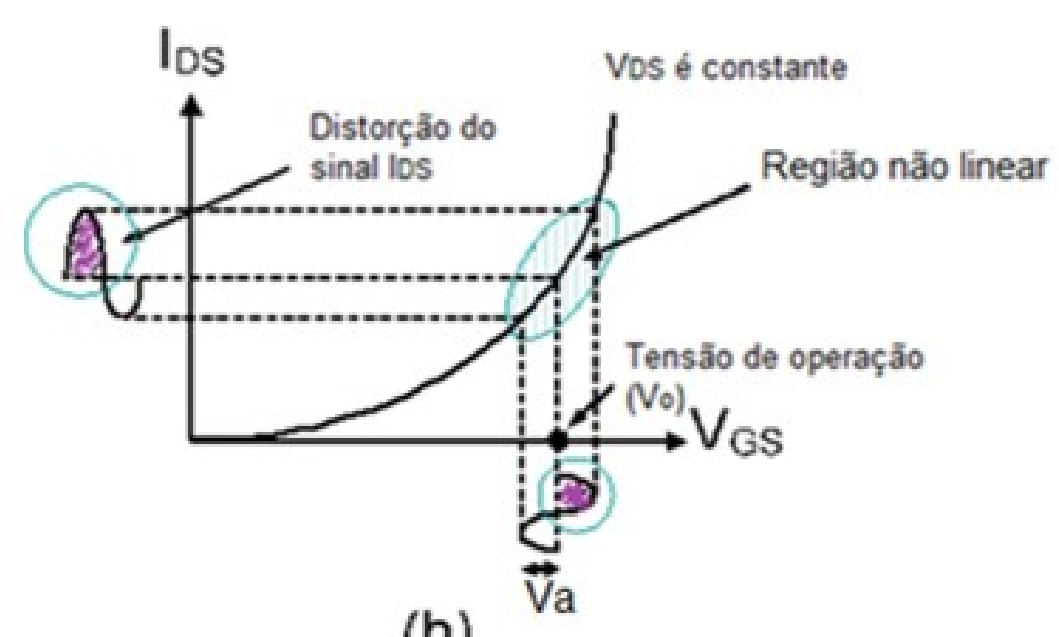

(b)

Fonte: Autor “Adaptado" de Dantas, 2008

Sendo, na Figura 13, VDD a tensão de alimentação.

\subsection{MÉTODOS PARA DETERMINAR A DISTORÇÃO HARMÔNICA EM MOSFETS}

Há três métodos mais conhecidos na literatura para poder calcular a distorção harmônica de sinais elétricos. São eles:

- Método da série de Fourier: Para poder aplicá-lo, é necessário realizar a caracterização elétrica dos dispositivos em corrente alternada $(\mathrm{AC})$, no qual o sinal de saída do dispositivo (que normalmente é visualizado no domínio do tempo) é transformado para o domínio da frequência, gerando uma distribuição espectral do sinal [24]. 
- Método da série de Taylor: Este método não necessita da caracterização elétrica em corrente alternada do circuito. Logo, torna-se primordial selecionar um ponto de polarização e o conhecimento da função de transferência do circuito. Todavia, a desvantagem desse método é a necessidade da realização de sucessivos cálculos de derivadas de ordem superior da função de transferência, tornando esse método muito suscetível a ruídos [23][24].

- Método da Função Integral (Integral Function Method, IFM): Este método considera apenas a característica de transferência da corrente contínua (DC), podendo ser aplicado tanto a circuitos quanto aos dispositivos isolados de qualquer tecnologia. É preciso uma análise geométrica da forma da curva de transferência para que se possa determinar a não-linearidade do objeto de estudo [25][26][27][28].

Como já visto em estudos anteriores, o IFM apresenta um número maior de vantagens com relação aos métodos das séries de Taylor e de Fourier. Diferentemente da série de Fourier, o IFM não necessita da caracterização em tensão alternada dos circuitos avaliados ou do cálculo das derivadas de ordens mais altas, como na série de Taylor [6][8]. Desta forma, este método apresenta maior imunidade à ruídos das medidas realizadas [6][25][26]. Ademais, o IFM dispensa informações em regime alternado de operação para caracterizar a distorção do componente ou do circuito a ser estudado [25][27][28].

O método IFM permite o cálculo de forma direta das principais figuras de mérito da distorção harmônica: a distorção harmônica total, distorções harmônicas de segunda ordem (HD2) e terceira ordem (HD3) [28].

Para poder explicar a metodologia do IFM, na perspectiva de usuário, consideramos um MOSFET operando como um amplificador de um único MOSFET e a curva IDS em função da $\mathrm{V}_{\mathrm{GS}}$ desse transistor operando na região de saturação [26]. O sinal de entrada do MOSFET é representado pela Equação (8) [28].

$$
X=X_{0}+A * \operatorname{sen}(\theta)
$$

sendo $\mathrm{X}$ o sinal de entrada, $\mathrm{X}_{0}$ o ponto de polarização DC para polarizar o MOSFET na região de saturação, A a amplitude do sinal de entrada e $\theta$ é igual ao produto $\omega . t$, no qual $\omega$ é a velocidade angular do sinal de entrada, dado por $2 \pi \mathrm{f}$.

O sinal de saída do MOSFET (Y) é expresso por uma função não-linear de $f(X)$, que é representada pela Equação (9) [28]: 


$$
Y=f(X)
$$

Considerando a curva IDS em função $V_{G S}$ não-linear dada por $Y=f(X)$ [28], deve-se selecionar a região de interesse dessa curva. Então, essa curva característica DC é normalizada em ambos os eixos [abscissas (x) e ordenadas (y)] no intervalo entre 0 e 1 , através das equações (10), (11) e (12), respectivamente [27][28].

$$
\begin{gathered}
x=\frac{X-\left(X_{0}-A\right)}{\left(X_{0}+A\right)-\left(X_{0}-A\right)}=\frac{X-\left(X_{0}-A\right)}{2 A} \\
y=\frac{Y(X)-Y\left(X_{0}-A\right)}{Y\left(X_{0}+A\right)-Y\left(X_{0}-A\right)} \\
X=X_{0}+A(2 x-1)
\end{gathered}
$$

A Figura 14 ilustra como é feita a normalização da curva característica IDS em função da $V_{G S}$ de um MOSFET, em que se aplica um sinal composto por uma componente DC e uma componente senoidal [8][28].

Figura 14 - Ilustração do processo de normalização de parte da curva característica IDs em função de $V_{G S}$ que é dado pelo método IFM.

\section{Curva normalizada}

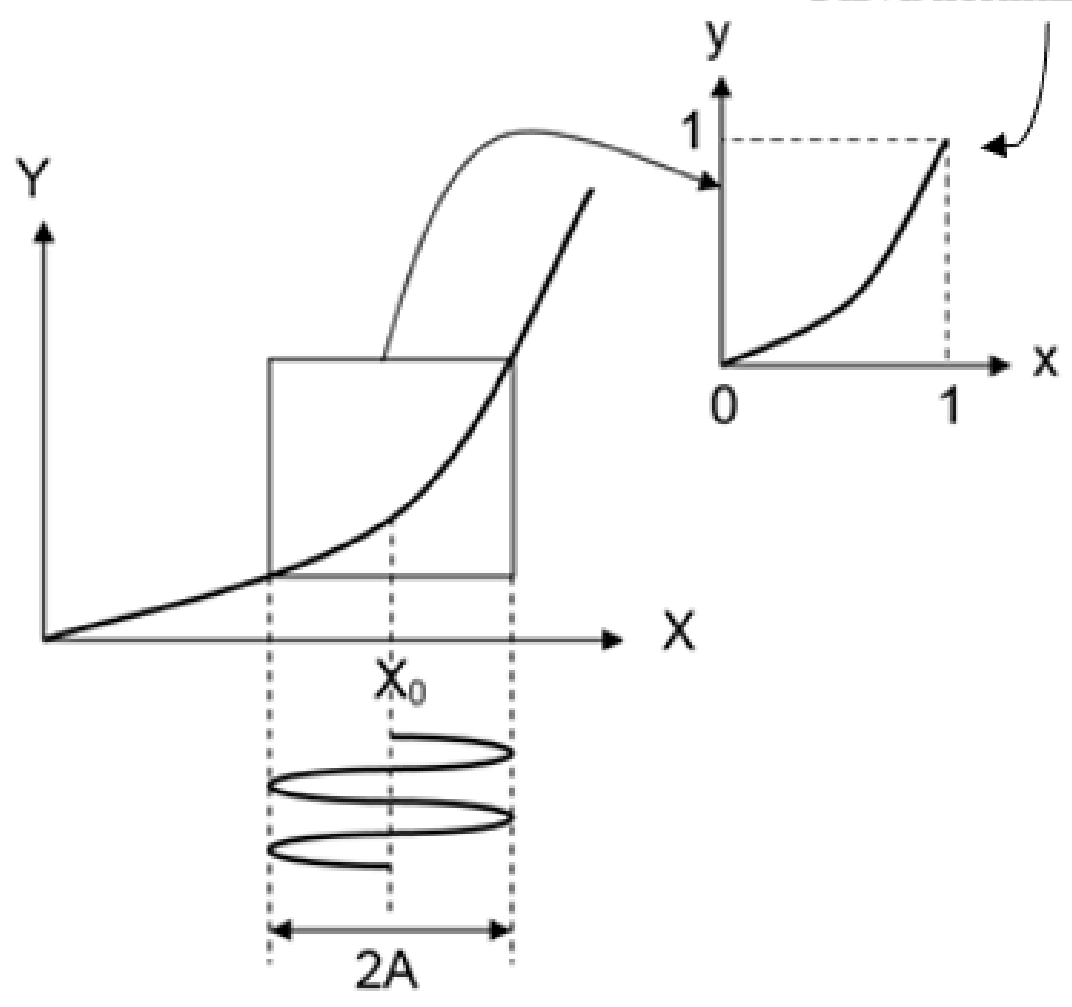

Fonte: Autor “Adaptado de” Dantas, 2007 
Ao substituir a Equação (12) na (11) obtém-se a equação (13), que expressa o sinal de saída $[\mathrm{y}(\mathrm{x})]$ normalizado em função de $\mathrm{X}_{0}$ e A [28].

$$
y(x)=\frac{Y\left(X_{0}+A(2 x-1)\right)-Y\left(X_{0}-A\right)}{Y\left(X_{0}+A\right)-Y\left(X_{0}-A\right)}
$$

Assim, o quadrado resultante da área igual a 1 é dividido em duas áreas, sendo que uma é a área superior (AREA2) e a outra é a área inferior (AREA1) que é resultado do processo de normalização, como pode ser visto na Figura 15 [25][26].

Figura 15 - Exemplo de uma curva característica IDs em função de $\mathrm{V}_{\mathrm{GS}}$ normalizada, em que são evidenciadas as áreas abaixo e acima da curva definido por $\mathrm{Y}=\mathrm{X}$.

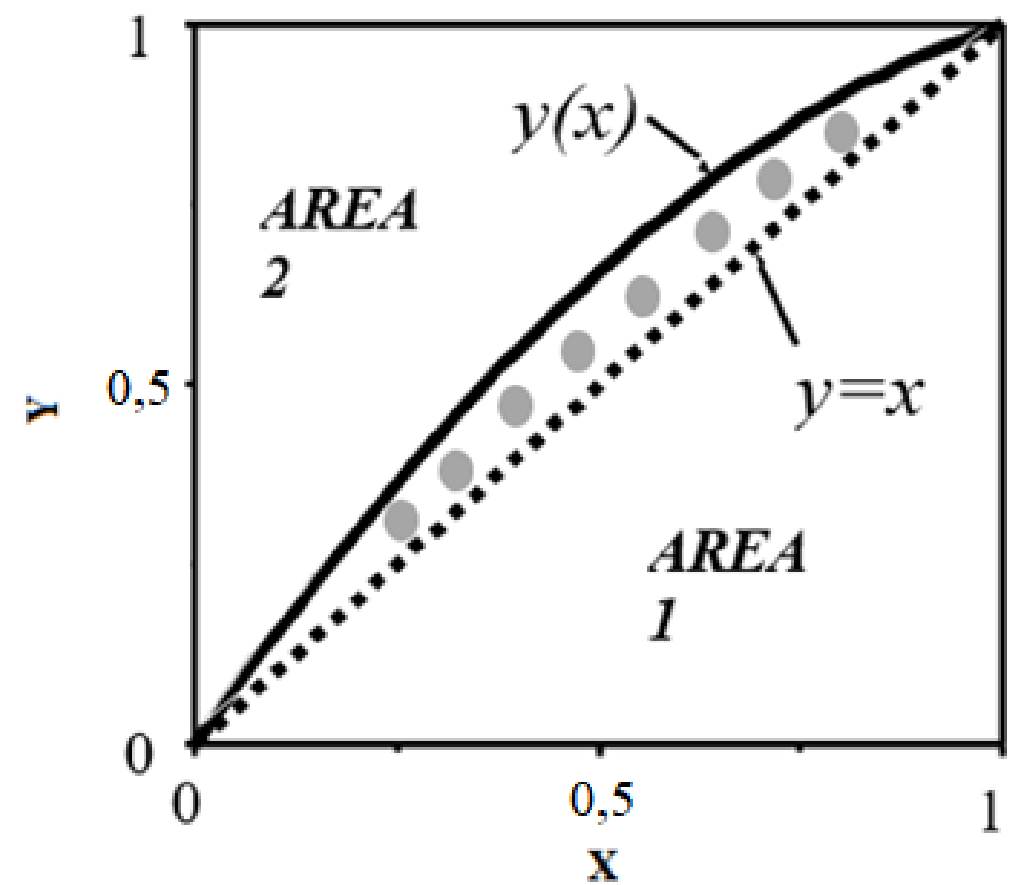

Fonte: Autor "Adaptado de" Cerdeira, 2008

Se a função $\mathrm{y}(\mathrm{x})$ fosse linear, a AREA 1 seria idêntica a AREA 2 e, consequentemente, $y(x)=x[25][26]$. A distorção harmônica está relacionada com a diferença entre as áreas abaixo (AREA 1) e acima (AREA 2) da função y(x) [28].

A área preenchida com círculos, na Figura 15, representa a diferença entre ambas as áreas (AREA 1 e AREA 2), sendo definida pela função D, que é dada pela Equação (14) [27]:

$$
D=|A ́ R E A 1-\hat{A} R E A 2|=\left|\int_{0}^{1} y(x) \cdot d x\right|-\left|\int_{0}^{1} y(x) \cdot d y\right|=\left|2 \cdot \int_{0}^{1} y(x) \cdot d x-1\right|
$$


Caso a curva característica DC apresente mais elementos que definam a não-linearidade (Ex: Na curva normalizada o sinal de saída passa mais de uma vez na bissetriz da curva DC), como ilustrado na Figura 16, a bissetriz da curva DC normalizada pode e deve ser cortada em mais de um ponto para poder compensar as não semelhanças entre as áreas (AREA 1 e AREA 2) [6][29].

Figura 16 - Exemplo de uma curva característica normalizada do MOSFET que intercepta a bissetriz em um ponto qualquer e a correção feita através da função ys(x) pelo método IFM para determinação da distorção harmônica

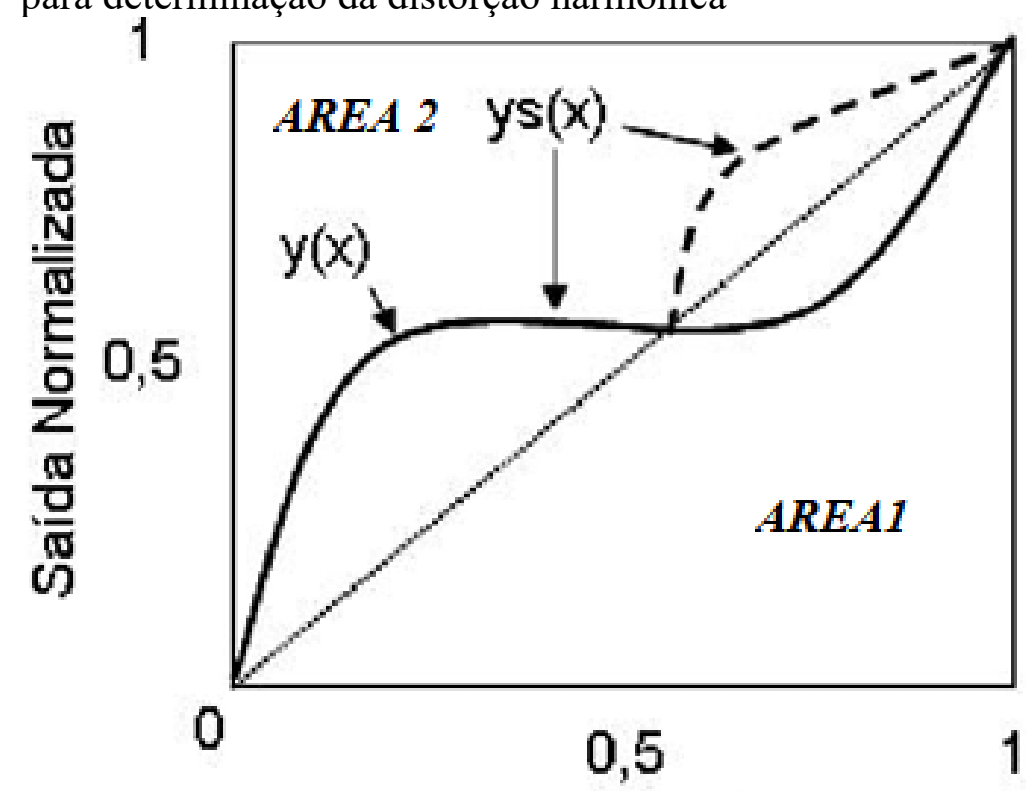

Entrada Normalizada

Fonte: Autor "Adaptado de" Doria, 2007

Para que esse aspecto não-linear possa ser levado em conta, é preciso introduzir uma nova função chamada de ys(x), que é dada pela Equação (15) [27][28].

$$
y s(x)=|y(x)-x|+x
$$

Em razão deste ajuste e para compensar as diferenças entre as áreas, uma nova função Ds é definida e é dado pela Equação (16) [28]:

$$
D_{s}=2 \cdot \int_{0}^{1} y s(x) \cdot d x-1
$$

O uso da função Ds evita a necessidade de calcular o ponto de intersecção entre a função $\mathrm{y}(\mathrm{x})$ e a bissetriz ilustrada no gráfico da Figura 16 [28]. 
Cabe ressaltar que, em muitos casos, o valor da distorção harmônica de terceira ordem (HD3) possui ordem de grandeza muito inferior a THD [28]. Em circuitos balanceados, as harmônicas de ordem par são suprimidas fazendo com que HD3 se torne representativa na THD [8][28].

Essa supressão das harmônicas pares é matematicamente expressa por meio da Equação (17) [28]:

$$
Y_{r}=y(x)-y(-x)
$$

Onde $Y_{\mathrm{r}}$ é normalizado da mesma forma que foi feito anteriormente [Equação (13)], obtém-se a função $\mathrm{y}_{\mathrm{r}}(\mathrm{x})$, que é dada pela Equação (18) [28]:

$$
y_{r}(x)=\frac{Y_{r}\left(X_{0}+A(2 x-1)\right)-Y_{r}\left(X_{0}-A\right)}{Y_{r}\left(X_{0}+A\right)-Y_{r}\left(X_{0}-A\right)}
$$

Assim, após a normalização do $\mathrm{Y}_{\mathrm{r}}$, têm-se uma nova função $\mathrm{D}$, que pode ser representado por $\mathrm{D}_{\mathrm{r}}$, que é calculada pela Equação (19) [28]:

$$
D_{r}=2\left|2 \int_{0}^{0,5} y_{r}(x) \cdot d x-(0,5)^{2}\right|=4 * \int_{0}^{0,5} y_{r}(x) \cdot d x-0,5
$$

Como já supracitado, é necessário considerar que essas funções normalizadas podem apresentar uma forte não-linearidade e, em função disto, dispor de múltiplos cruzamentos com a bissetriz da curva característica DC [27][28]. Para poder corrigir esse efeito no $\mathrm{y}_{\mathrm{r}}(\mathrm{x})$, é definida a função yrs(x), dada pela Equação (20) [28]:

$$
y_{r s}(x)=\left|y_{r}(x)-x\right|+x
$$

Que por sua vez, deve determinar uma nova função de $\mathrm{D}_{\mathrm{r}}$ a partir de $\mathrm{y}_{\mathrm{rs}}(\mathrm{x})$, determinando a nova expressão de Drs, apresentada pela Equação (21) [28]:

$$
D_{r s}=2 \int_{0}^{1} y_{r s}(x) \cdot d x-1
$$

Após determinar todas as quatro funções integrais, o passo seguinte consiste, sobretudo, em definir a distorção harmônica tendo como suporte estas funções [27][28]. 
Vale ressaltar, nesse momento, a definição da distorção harmônica. A distorção harmônica de ordem n (HDn) é definido pela equação (22), com n valendo $0,2,3, \ldots$ [25].

$$
H D n=\frac{\left|C_{n}\right|}{\left|C_{1}\right|}
$$

Sendo $C_{1}$ igual a amplitude de saída da frequência na fundamental e $C_{n}$ a amplitude de saída da harmônica de ordem $n$.

Logo, a distorção harmônica total, incluindo a componente DC do sinal de entrada ( $\mathrm{THD}_{0}$ ), é determinada a partir da raiz quadrada da soma dos quadrados dos harmônicos de ordem n [28]. Ressaltando, são predominantes as harmônicas de segunda e terceira ordem e, por essa razão, o THDo pode ser expresso pela Equação (23) [28].

$$
T H D_{0}=\sqrt{H D 0^{2}+H D 2^{2}+H D 3^{2}}
$$

Sendo HD0 a distorção harmônica correspondente à componente DC do sinal de entrada, HD2 a distorção do harmônico de segunda ordem e o HD3 a distorção harmônica de terceira ordem [28].

No caso de uma distorção harmônica fraca (Sinal com pouca distorção), a distorção da componente DC passa a ser praticamente igual àquela gerada pelos harmônicos pares, sendo que o HD2 é responsável quase que pela totalidade desta componente, posto que, os harmônicos de ordem mais alta acabam se tornando desprezíveis, resultando na expressão fornecida pela Equação (24) [26][27][28].

$$
H D 2 \approx \frac{3}{4} * D
$$

No caso da distorção do terceiro harmônico, pode ser aproximada pela função Dr [28], visto que esta função integral suprime os harmônicos pares, tornando a HD3 a principal harmônica no sinal resultante, obtendo assim, a expressão provida pela Equação (25) [25][26].

$$
H D 3 \approx D_{r}
$$

Portanto, a distorção harmônica total (THD) pode ser descrita como a $\mathrm{THD}_{0}$, excluindo a componente DC e, resultando na Equação (26) [28]. 


$$
T H D=\sqrt{H D 2^{2}+H D 3^{2}}
$$

Tendo como suporte as equações (23), (24), (25) e (26), uma nova equação em função de $\mathrm{D}_{\mathrm{s}}$ e de $\mathrm{D}_{\mathrm{rs}}$ foi desenvolvida para a obtenção de THD, de acordo com expressão da Equação (27) [25].

$$
T H D=\sqrt{(0,75 * D)^{2}+\left(D_{r}\right)^{2}}
$$

As funções Ds e Drs podem ser analisadas de forma análoga às funções D e Dr quando as considerações de distorção harmônica fraca não forem válidas [25]. Nesses casos, os cálculos das figuras de mérito de distorções harmônicas, como o THD, HD2 e HD3, podem ter seus valores de D e Dr substituídos por Ds e Drs [28].

Como usualmente os MOSFETs apresentam diferentes valores de VEA e diferentes Av, para uma mesma tensão de polarização $V_{D S}$, é necessário realizar a normalização de THD em função de Av [26][27].

\subsection{GEOMETRIAS DE PORTA NÃO CONVENCIONAIS PARA O MOSFET}

O intuito de estudar as novas geometrias de porta (Diamante, OCTO e Fish) se deve ao fato de constituir novas possibilidades de sobrevida para a tecnologia planar de fabricação de CI CMOS quando comparado ao desempenho elétrico e interferências às radiações ionizantes, face à tecnologia tridimensional de MOSFETs a um custo zero de produção de CIs CMOS [13].

O MOSFET do tipo Diamante (DM) possui geometria de porta hexagonal [13][29], enquanto o CM possui geometria de porta retangular. Ao modificar a geometria de porta retangular para hexagonal de um MOSFET, é possível aumentar o seu campo elétrico longitudinal, no canal em relação a do CM equivalente [13], refletindo no aumento de sua corrente entre o dreno e a fonte [13][29], na sua transcondutância [13][29], na sua razão gm/IDS, no seu ganho de tensão intrínseco [30] e na sua frequência de ganho de tensão unitário (fT) [31], entre outros, admitindo-se que esses transistores têm as mesmas condições de polarização, área de porta ou mesma razão de aspecto.

A ideia da criação do MOSFET do tipo Diamante foi de usar o efeito de canto na direção longitudinal do canal do MOSFET, denominado de "Efeito de Canto Longitudinal" para 
potencializar o campo elétrico longitudinal resultante ao longo do canal. Dessa forma, ao aumentar a velocidade média de deriva dos portadores móveis do canal ( $v=\mu * \vec{\varepsilon}_{/ /}$, sendo $\mathrm{V}$ a velocidade média de deriva dos portadores do canal, $\mu$ a mobilidade dos portadores móveis do canal e $\overrightarrow{\varepsilon_{/ /}}$o campo elétrico longitudinal resultante na região de canal), é possível ter um maior desempenho elétrico dos seus principais parâmetros elétricos e figuras de mérito [13][29][30][31].

A Figura 17 ilustra como é dado o aumento do campo elétrico longitudinal na região de canal do DM decorrente ao LCE em uma vista simplificada do DM, onde também mostra as componentes do campo elétrico longitudinal [13].

Figura 17 - Vista superior simplificada do leiaute de um MOSFET do tipo Diamante mostrando as suas componentes do campo elétrico longitudinal, sua resultante e as características dimensionais.

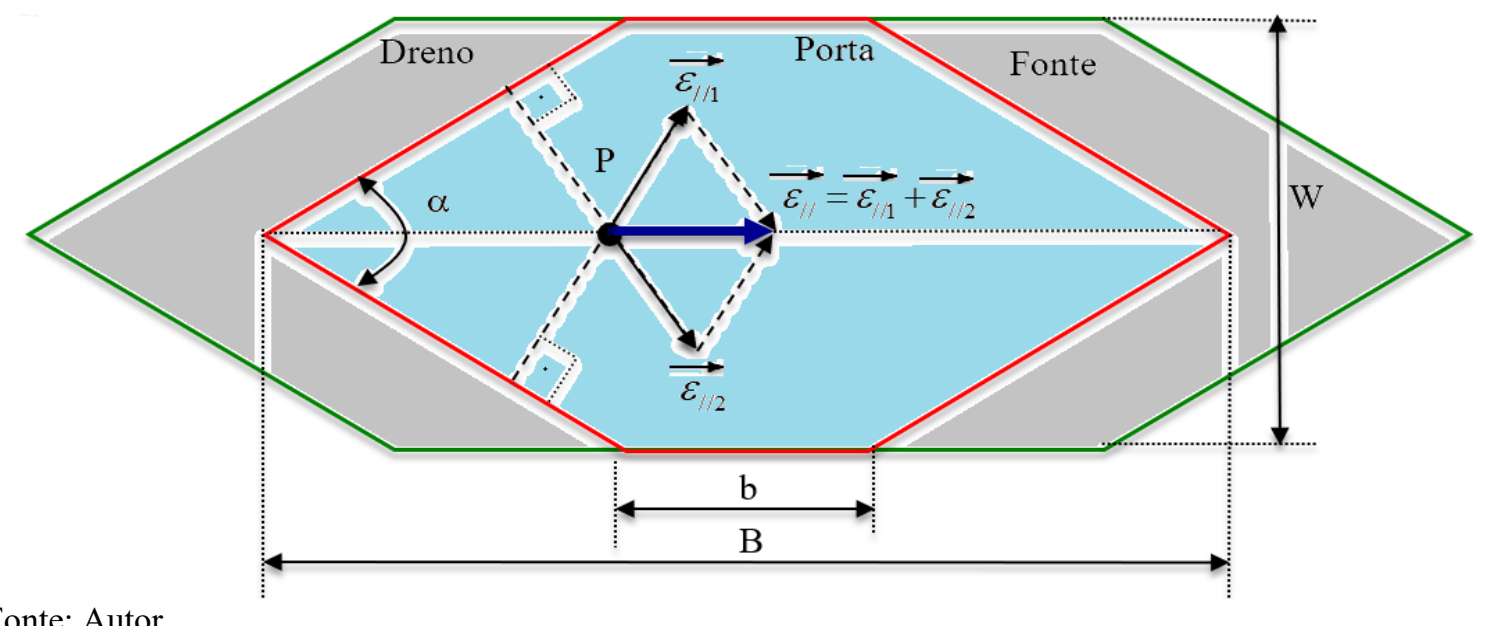

Na Figura 17, $\alpha$ representa o ângulo de abertura da geometria hexagonal de porta do DM, devido a forma geométrica hexagonal de porta, , $\overrightarrow{\varepsilon / / 1}$, e $\overrightarrow{\varepsilon_{/ / 2}}$ são as componentes do campo elétrico longitudinal ao longo do canal no ponto $\mathrm{P}$, sendo perpendiculares às interfaces formadas pelas regiões de dreno e canal no ponto $\mathrm{P}$, decorrente da aplicação da $\mathrm{V}_{\mathrm{DS}}$, B e b são os comprimentos de canal da porta de formato hexagonal, não convencional, maior e menor, respectivamente, e $\mathrm{W}$ é a largura de canal do transistor.

Outra decorrência que surge a partir dessa nova proposta de geometria de leiaute de porta é o efeito da associação paralela de MOSFETs com diferentes comprimentos de canal [13][29][30]. Esse efeito adicional tem a capacidade de potencializar a sua corrente elétrica entre o dreno e a fonte, uma vez que o MOSFET com geometria de porta hexagonal pode ser considerado como uma associação paralela de infinitos MOSFETs com leiaute de porta 
retangular de diferentes comprimentos de canal e com larguras de canal infinitesimais. Assim sendo, a corrente entre dreno e fonte tende a fluir mais pelas bordas do dispositivo, onde existem os MOSFETs com menores comprimentos de canal [13][29][30].

A Figura 18 ilustra o DM dividido em infinitos MOSFETs com geometria de porta retangulares, além das distintas dimensões de canal $\left(\mathrm{b} \leq \mathrm{L}_{\mathrm{i}} \leq \mathrm{B}\right.$, onde $\mathrm{L}_{\mathrm{i}}$ representa os diferentes comprimentos de canal da estrutura hexagonal de porta e i é definido como um número inteiro) e com larguras de canal infinitesimais [33].

Figura 18 - Representação física do MOSFET do tipo Diamante construído de infinitos MOSFETs com geometria de porta retangular associados em paralelo.

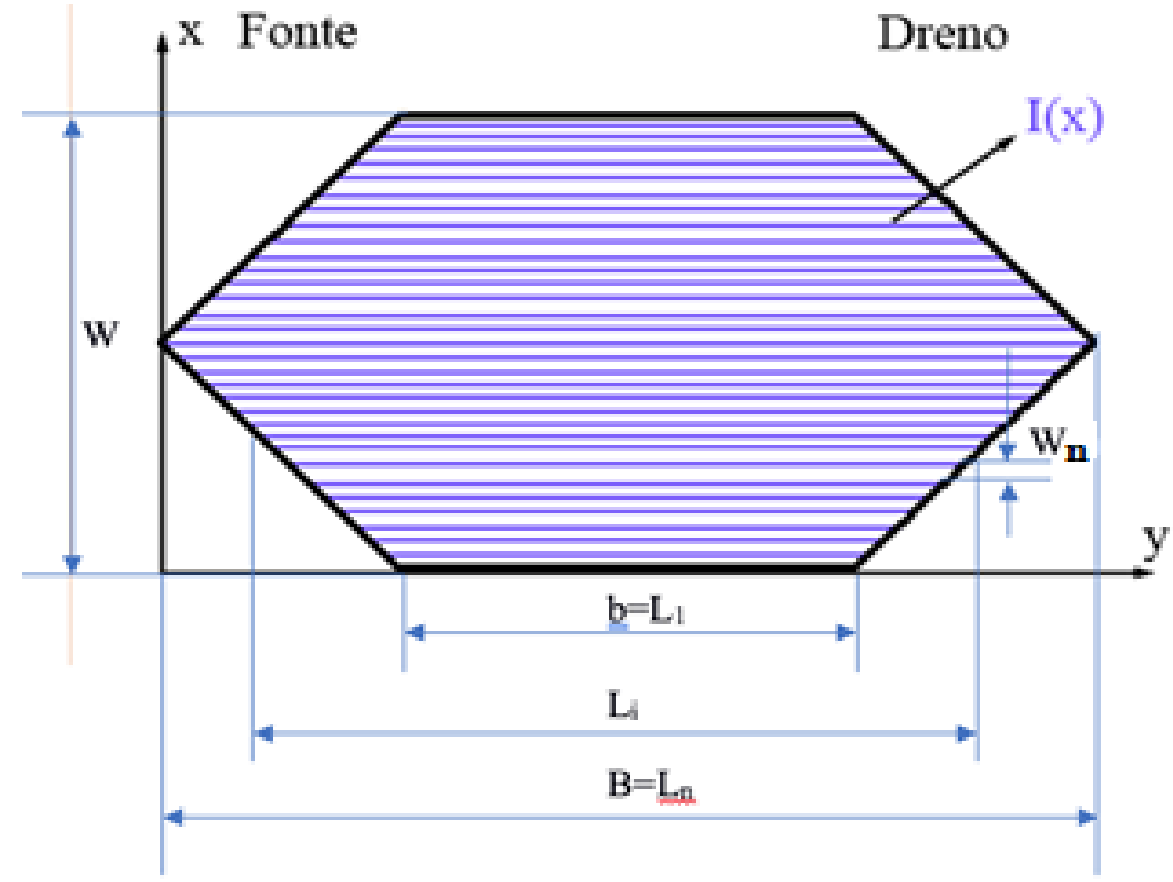

Fonte: Autor "Adaptado de" Gimenez, 2014

Na Figura 18, I(x) representa a corrente elétrica entre o dreno e a fonte correspondente a cada MOSFET de porta retangular que compõem o MOSFET do tipo Diamante, $\mathrm{W}_{\mathrm{n}}$ é a largura de canal infinitesimal, sendo n um número inteiro tendendo a infinito. Ademais, $\mathrm{L}_{1}, \mathrm{~L}_{2}, \ldots, \mathrm{L}_{\mathrm{i}}$, ..., $\mathrm{L}_{\mathrm{n}}$ são os diferentes comprimentos de canal do MOSFET do tipo Diamante.

O correspondente circuito elétrico equivalente do dispositivo visto na Figura 18 é ilustrado na Figura 19 [33]. 
Figura 19 - Correspondente circuito elétrico equivalente de um DM.

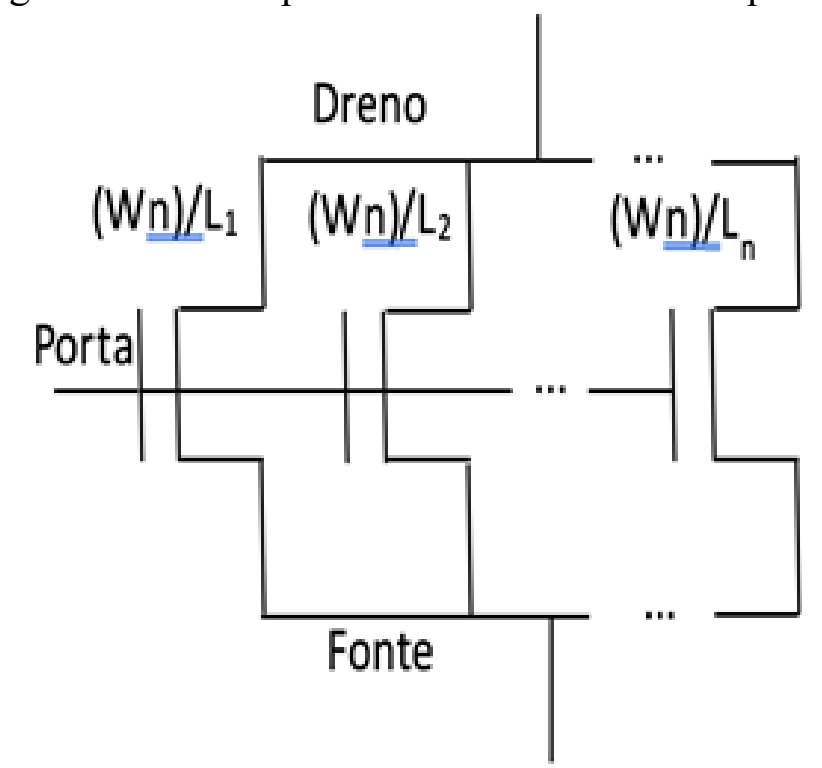

Fonte: Autor “Adaptado de” Gimenez, 2014

Ao equacionar o comprimento efetivo de canal do DM, verifica-se que ele tem a capacidade de reduzir o comprimento efetivo de canal ( $\mathrm{L}_{\text {eff }}$ ) dos MOSFET do tipo Diamante em relação ao comprimento de canal de um CM equivalente, considerando-se que eles apresentam a mesma área de porta $\left(\mathrm{A}_{\mathrm{G}}\right)$ e mesma largura de canal. A Equação (28) define o valor do comprimento de canal efetivo do MOSFET do tipo Diamante [13].

$$
L_{e f f}=\frac{(B-b)}{\ln \left(\frac{B}{b}\right)}
$$

O terceiro efeito que surgiu com a aplicação dessa técnica de leiaute é o "Efeito da Desativação dos MOSFETs Parasitários das Regiões de Bico de Pássaro" (Deactivation of Parasitic MOSFETs of the Bird's Beak Regions Effect, DEPAMBBRE) [33]. Esse efeito gera linhas de campo elétrico longitudinais, curvas entre as regiões de bico de pássaro dos DMs, que existem intrinsecamente entre as regiões de fonte e dreno, sendo capaz de desativar eletricamente os MOSFETs parasitários dessas regiões como indica a Figura 20 [33]. 
Figura 20 - Linhas de campo elétrico longitudinais resultantes curvas entre as regiões de Dreno/Fonte e Canal do MOSFET do tipo Diamante (geometria de porta hexagonal) para um VDS maior do que $0 \mathrm{~V}$.

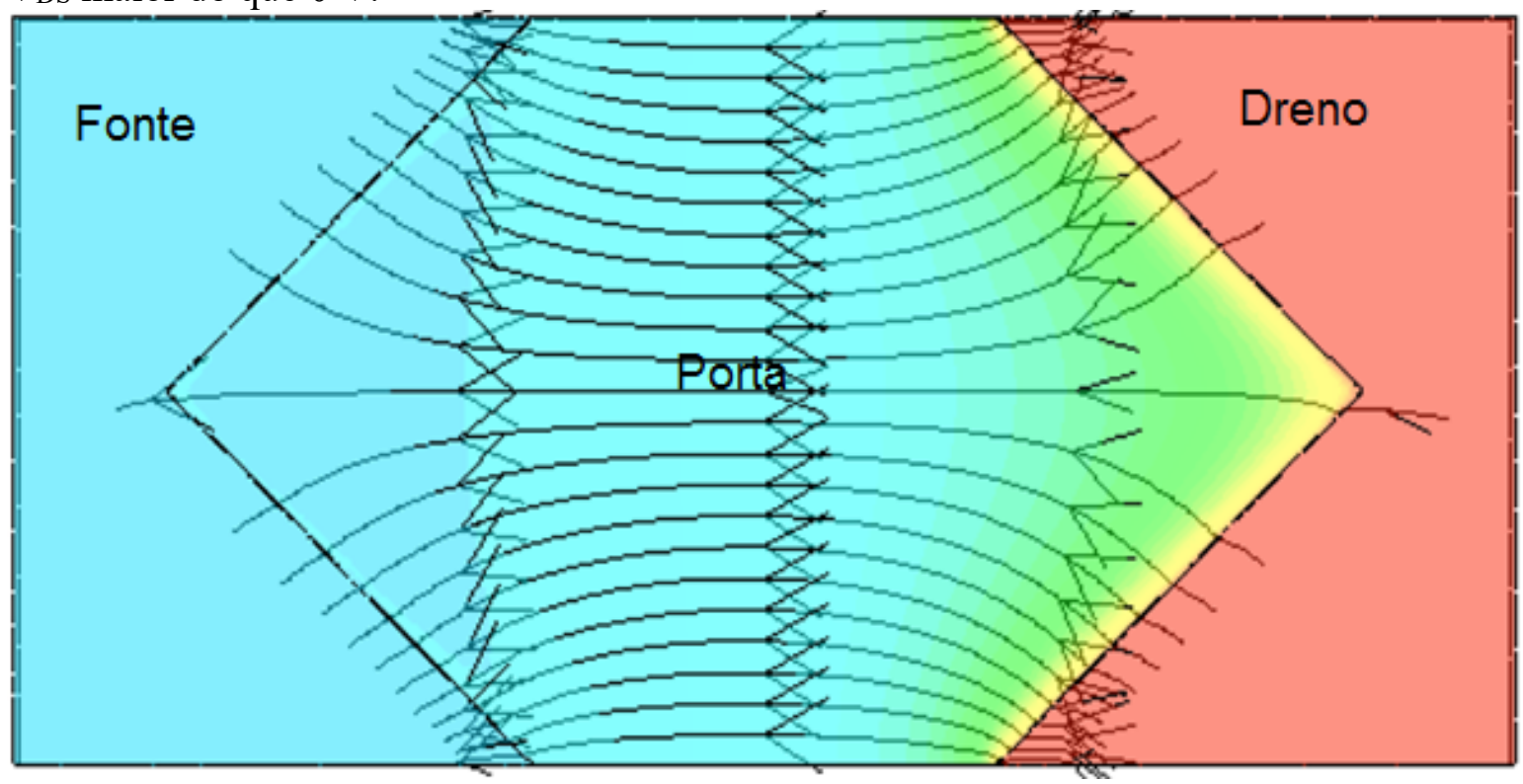

Fonte: Autor "Adaptado de" Gimenez, 2012

Nos MOSFETs de leiaute de porta retangular, esses transistores parasitários prejudicam o funcionamento dos dispositivos quando são submetidos às radiações ionizantes, como por exemplo, em aplicações espaciais de CIs CMOS [33]. Isso acontece em razão das radiações ionizantes que são responsáveis por reduzir a tensão de limiar desses MOSFETs parasitários, iniciando o seu funcionamento antes do transistor principal, e, consequentemente, tornando-se responsáveis por provocar um aumento da corrente de fuga (ILEAK) e da corrente de dreno de estado desligado (IoFF) do MOSFET principal [32][33].

\subsection{MOSFET DO TIPO DIAMANTE HÍBRIDO}

Em virtude das regras de projeto das tecnologias de fabricação de CIs CMOS mais recentes e sofisticadas não permitirem a fabricação de MOSFETs com geometrias de porta não convencionais que apresentam a região de porta do MOSFET não ortogonal à região ativa do dispositivo, foi proposto um inovador estilo de leiaute de MOSFET com geometria de porta não-convencional em paralelo com dois MOSFETs de geometria de porta retangular, criando assim uma nova forma de leiaute, o MOSFET de geometria de porta não convencional Híbrido (Hybrid MOSFET, HM). Para poder usufruir os ganhos que o DM proporciona [13][29][30][31] nessa tecnologia, foi implementado o MOSFET do tipo Diamante Híbrido, conforme está ilustrado na Figura 21. 
Figura 21 - Vista superior do leiaute de um nMOSFET de geometria de porta hexagonal (DM) em paralelo com dois nMOSFETs com porta retangular $\left(\mathrm{CM}_{1}\right)$.

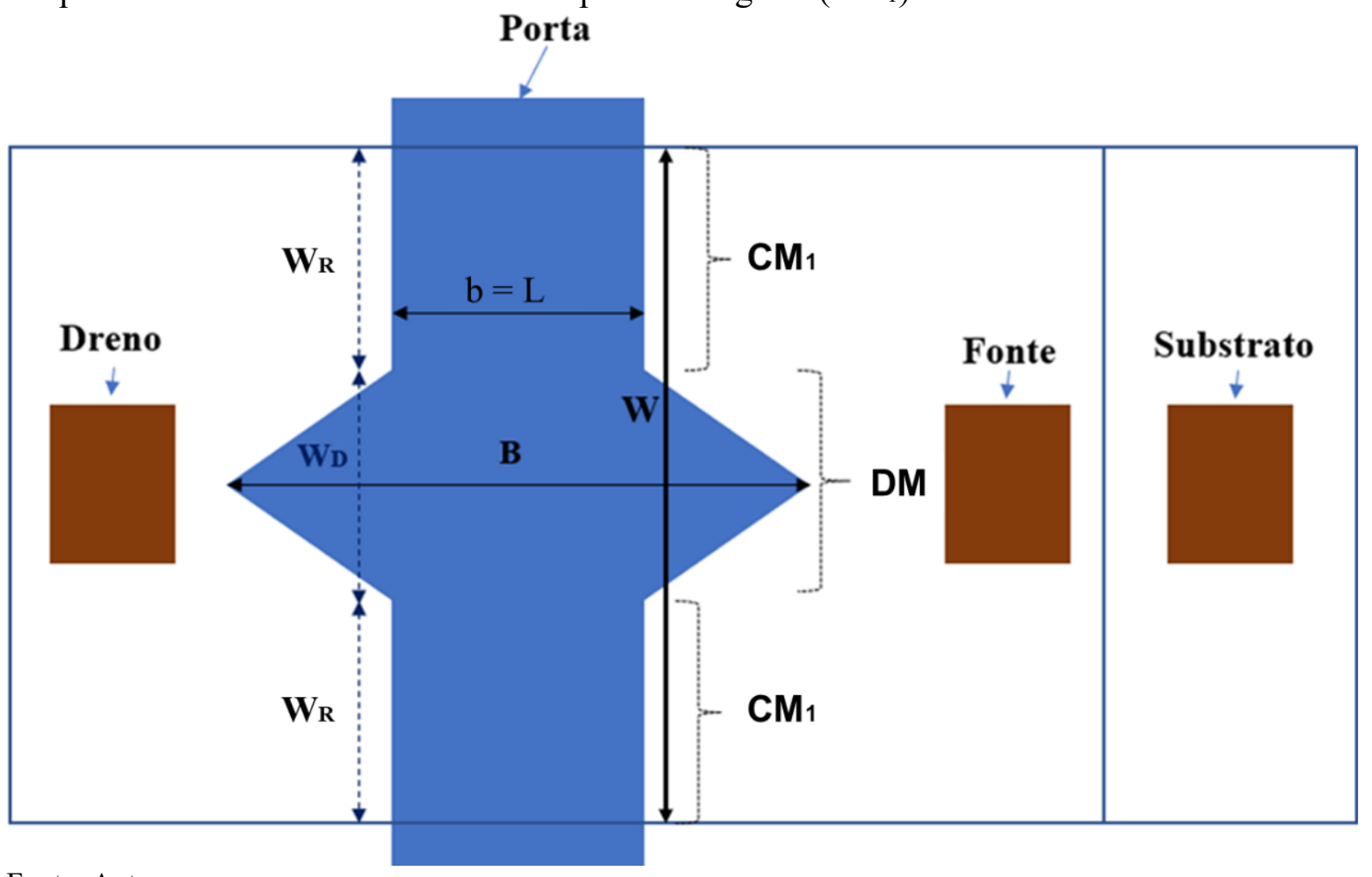

Fonte: Autor

Sendo L o comprimento de canal do MOSFET retangular, WD é a largura de canal do MOSFET não convencional (Diamante) e $W_{R}$ é a largura de canal do MOSFET de leiaute de porta retangular.

Num projeto de CI CMOS analógico são determinadas as razões de aspecto de todos os MOSFETs do circuito [1]. Essas dimensões sempre consideram geometrias de porta retangulares, dessa forma para transformar um MOSFET do tipo retangular num HDM, considerando que eles possuem as mesmas áreas de porta, é necessário seguir algumas etapas que estão ilustradas na Figura 22. 
Figura 22 - Processo de transformação de um MOSFET convencional obtido de um projeto de CI CMOS analógico num HDM: Leiaute simplificado de um MOSFET convencional (a). MOSFET convencional híbrido implementado com um MOSFET retangular principal e dois MOSFETs secundários (b) e o HDM, onde o MOSFET principal é substituído por um DM equivalente de mesma área de porta.

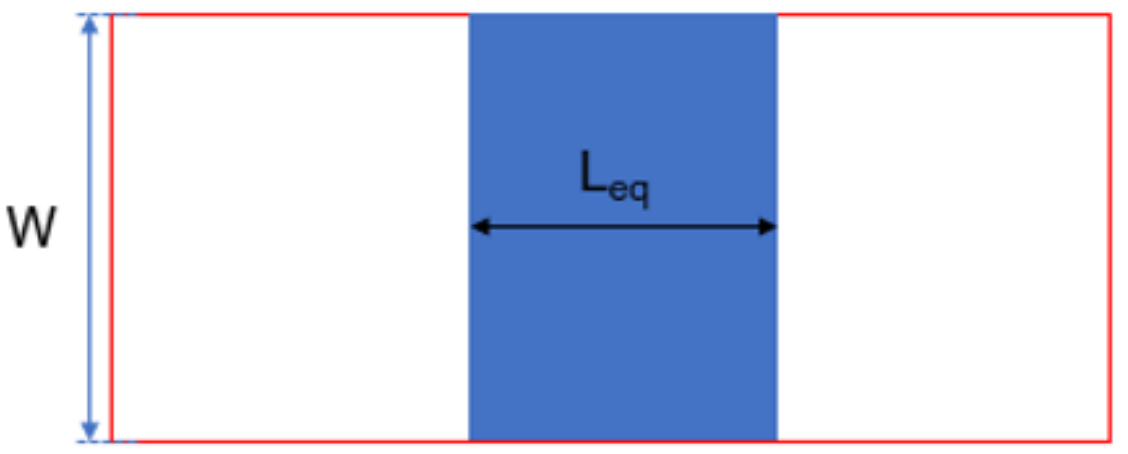

(a)

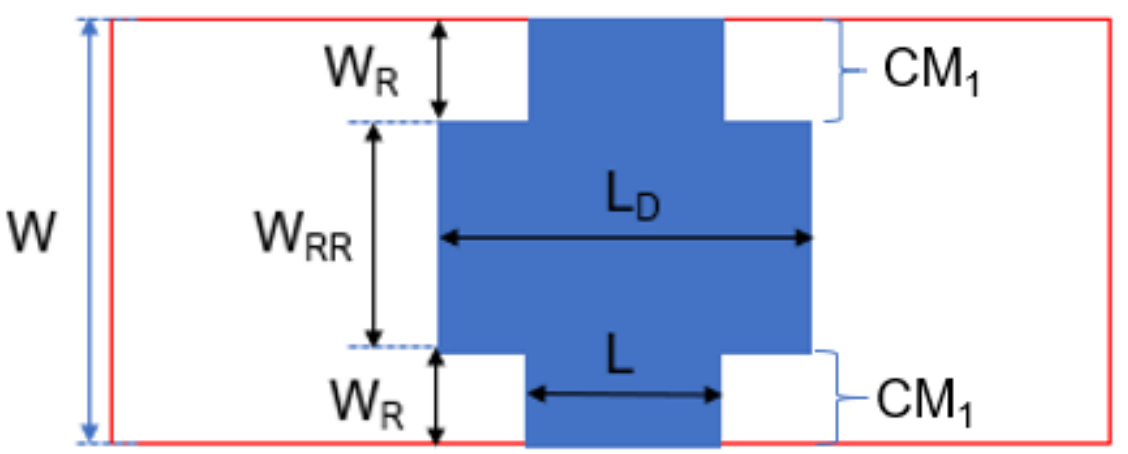

(b)

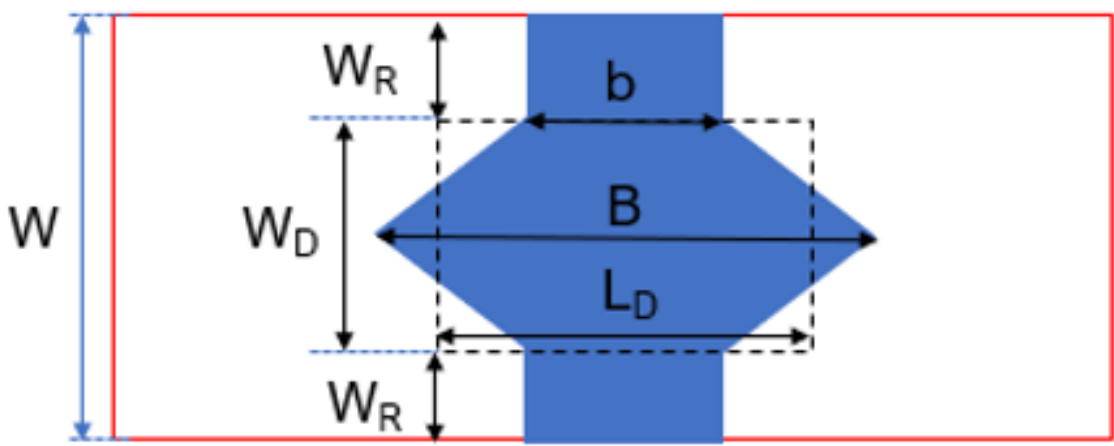

(c)

Fonte: Autor

Na Figura 22.(b), $W_{R R}$ é a largura de canal do MOSFET de porta retangular principal do circuito e o LD é o comprimento de canal do MOSFET de porta retangular principal do circuito. O LD é o comprimento de canal do MOSFET de porta retangular que apresenta a mesma área de porta que o MOSFET do tipo Diamante, que é calculado por intermédio da Equação (29) [13].

$$
L_{D}=\frac{B+b}{2}
$$


Abaixo é dada a descrição detalhada acerca das etapas de transformação de um CM para um DM que foram ilustradas na Figura 22.

a) Na primeira etapa é apresentado o MOSFET de porta retangular utilizado no projeto de CI CMOS analógico;

b) Mantendo a área de porta, é feito a mudança na estrutura do leiaute e como resultado se tem dois MOSFETs de porta retangular próximos a área ativa do dispositivo e um outro MOSFET de porta retangular no centro;

c) Com o MOSFET no centro da configuração dada pela Figura 22.(b) tem a mesma área de porta que um DM, o mesmo pode ser substituído pelo DM para que assim possa usufruir dos ganhos que esse transistor de leiaute de porta diferenciado proporciona em relação ao CM, obtendo dessa forma a configuração do HDM.

Para poder determinar a razão de aspecto do HDM, é necessário determinar o circuito elétrico equivalente do HDM apresentado na Figura 21, que está ilustrado na Figura 23.

Figura 23 - Circuito elétrico equivalente de um MOSFET de geometria de porta Diamante Híbrido contendo três MOSFETs, sendo um de geometria hexagonal e outros dois com geometrias de porta retangular.

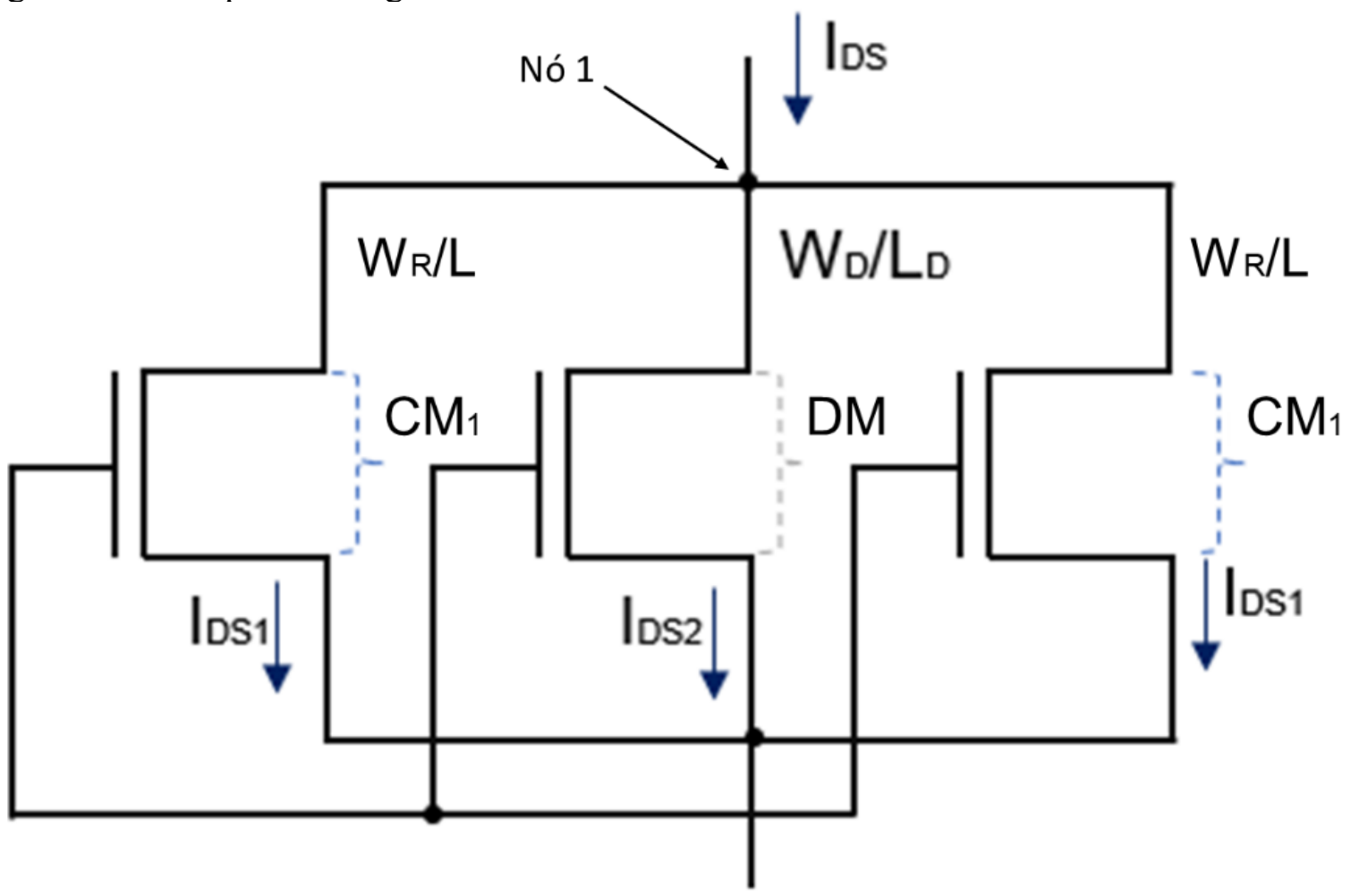

Fonte: Autor 
Sendo IDS1 a corrente entre o dreno e a fonte do MOSFET de porta retangular paralelo, IDS2 a corrente entre dreno e fonte do MOSFET com geometria de porta não convencional (hexagonal).

A razão de aspecto do HDM [(W/L)HDM] é obtido pela aplicação da primeira lei de Kirchoff no Nó 1 do circuito elétrico equivalente, ilustrado na Figura 23 [Equações (30) a (33)].

$$
\begin{aligned}
& I_{D S}=2 \cdot I_{D S 1}+I_{D S 2} \\
& \frac{1}{2} \cdot \mu_{n} \cdot C_{O X} \cdot\left(\frac{W}{L}\right)_{H D M} \cdot\left(V_{G S}-V_{T H}\right)^{2}=\frac{2}{2} \cdot \mu_{n} \cdot C_{O X} \cdot\left(\frac{W_{R}}{L}\right) \cdot\left(V_{G S}-V_{T H}\right)^{2}+\frac{1}{2} \cdot \\
& \mu_{n} \cdot C_{O X} \cdot\left(\frac{W_{D}}{L_{D}}\right) \cdot\left(V_{G S}-V_{T H}\right)^{2} \\
& \frac{1}{2} \cdot\left(\frac{W}{L}\right)_{H D M}=\left(\frac{W_{R}}{\mathrm{~L}}\right)+\frac{1}{2} \cdot\left(\frac{W_{D}}{L_{D}}\right) \\
& \left(\frac{W}{L}\right)_{H D M}=2 \cdot\left(\frac{W_{R}}{\mathrm{~L}}\right)+\left(\frac{W_{D}}{L_{D}}\right)
\end{aligned}
$$

Cabe ressaltar, para fins de comparação entre os HDMs e o CM equivalente a IDs do HDM será normalizado em função da razão de aspecto dada pela Equação (33). 


\section{ESTUDO EXPERIMENTAL COMPARATIVO ENTRE MOSFETS DOS TIPOS DIAMANTE HÍBRIDO E CONVECIONAL}

Neste capítulo apresentamos as características físicas dos MOSFETs estudados [Leiaute de porta Diamante Híbrido com diferentes ângulos $\alpha\left(45^{\circ}, 90\right.$ e $\left.135^{\circ}\right)$ e o de porta retangular] bem como os resultados experimentais das figuras de mérito mais importantes, tais como: a tensão de limiar, a transcondutância, a curva IDs/(W/L) em função do $\mathrm{V}_{\mathrm{GT}}$, a resistência série, a curva IDS/(W/L) em função de $V_{D S}$, a tensão Early, o ganho de tensão intrínseco, a inclinação de sublimiar, a corrente de estado ligado entre o dreno e a fonte, a corrente entre o dreno e a fonte de estado desligado e a distorção harmônica.

As curvas de IDS em função de $V_{G S}$ e IDS em função de $V_{D S}$ dos MOSFETs pesquisados foram extraídas à temperatura ambiente com o auxílio do equipamento de medição de curvas de CIs encapsulados LR: 8028 da Keithley.

\subsection{CARACTERÍSTICAS DIMENSIONAIS DOS MOSFETS UTILIZADOS PARA REALIZAR O ESTUDO EXPERIMENTAL}

Os circuitos integrados utilizados nesse projeto de pesquisa foram fabricados com tecnologia de fabricação de CIs CMOS convencional (Bulk) de $180 \mathrm{~nm}$ da TSMC. Os chips foram fabricados em Taiwan, através do programa universitário da Mini@asic do IMEC, Bélgica.

Nas figuras da Figura 24 a Figura 27 é possível visualizar os leiautes dos MOSFETs com porta retangular e dos MOSFETs dos tipos Diamante Híbrido com ângulos de abertura $\alpha$ iguais a $45^{\circ}, 90^{\circ}$ e $135^{\circ}$, respectivamente. 
Figura 24 - Leiaute do MOSFET do tipo retangular feito no software ICStation.

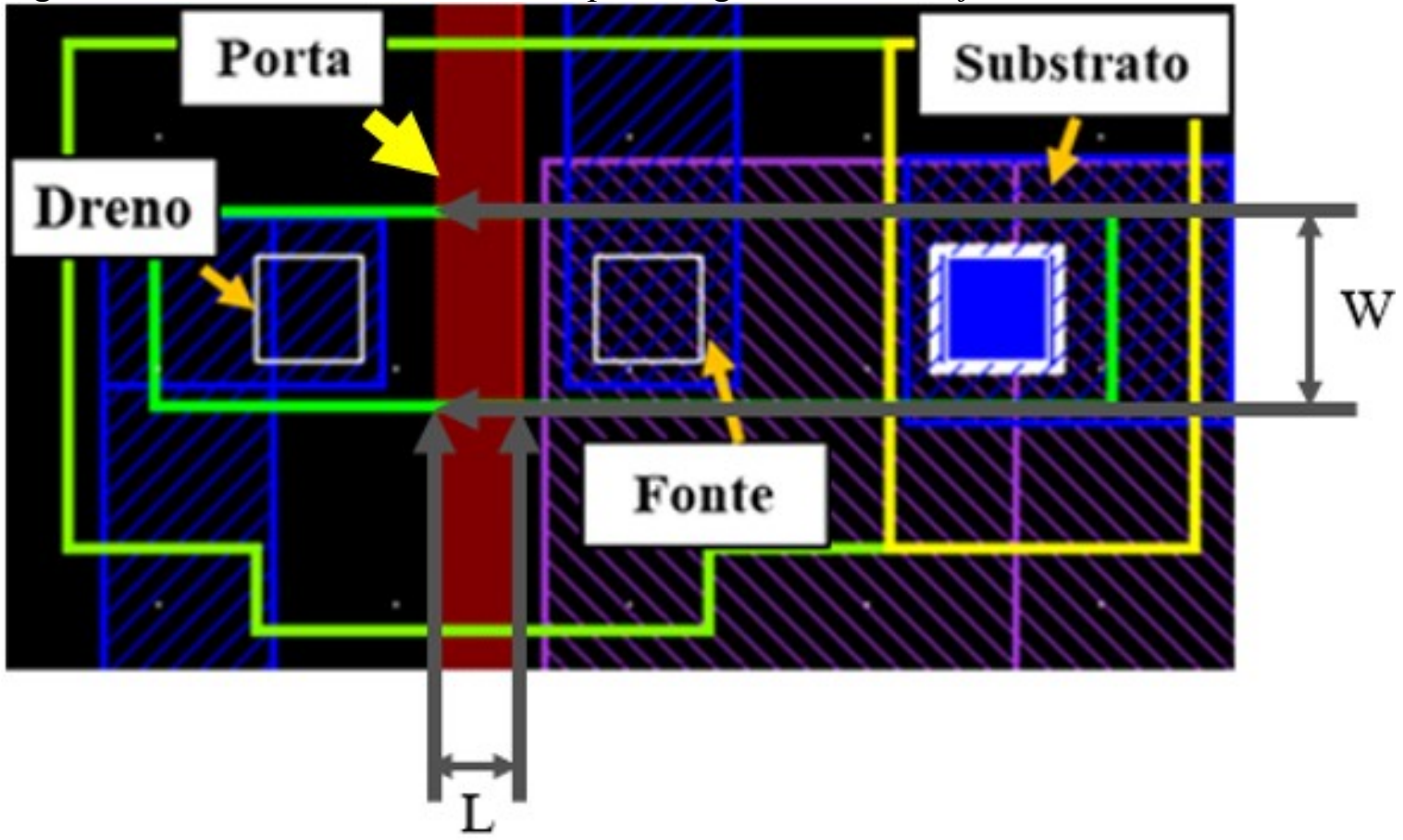

Fonte: Autor

Figura 25 - Leiaute do MOSFET do tipo Diamante Híbrido com ângulo $\alpha$ de $45^{\circ}$ feito no software ICStation.

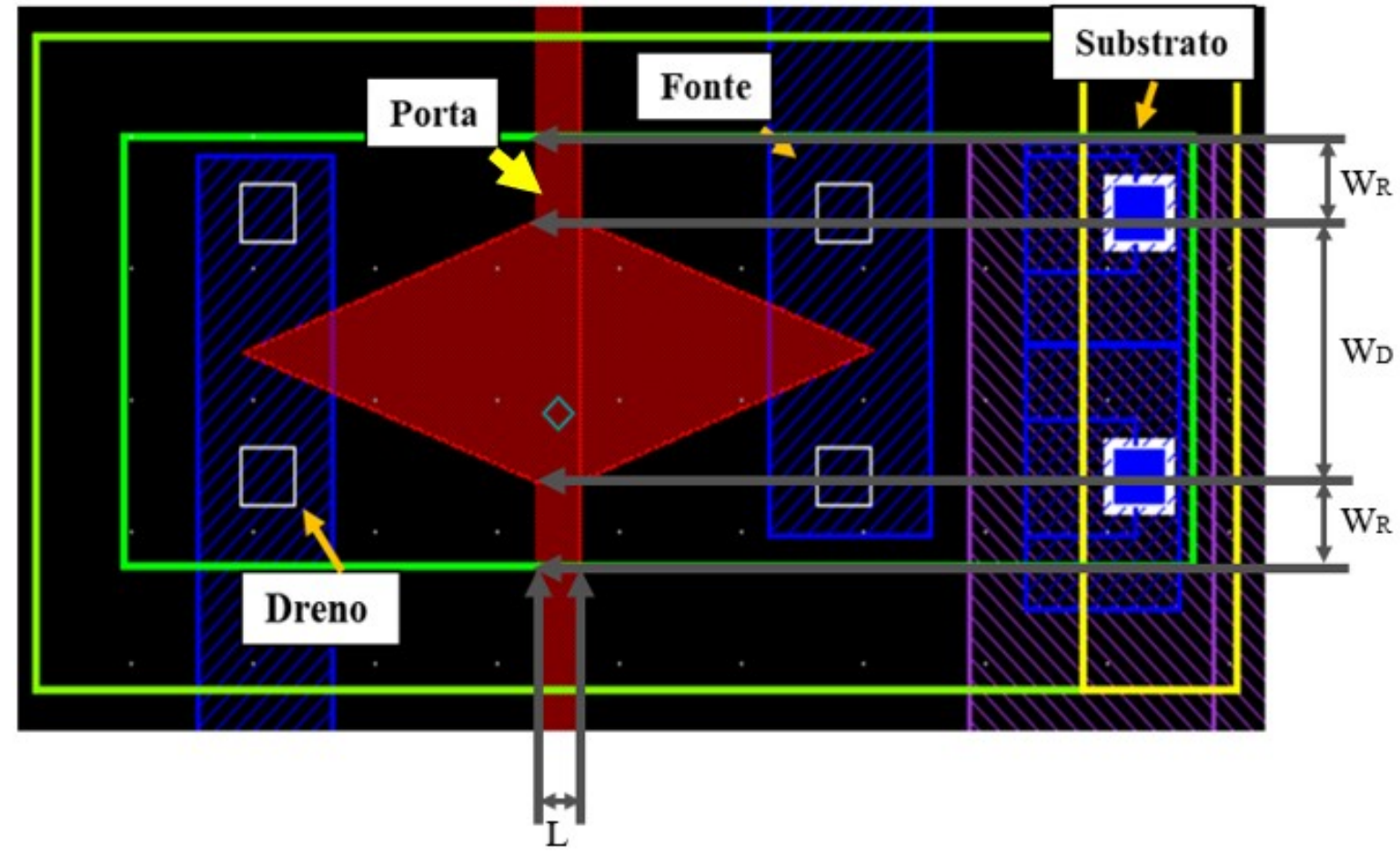

Fonte: Autor 
Figura 26 - Leiaute do MOSFET do tipo Diamante Híbrido com ângulo $\alpha$ de $90^{\circ}$ feito no software ICStation.

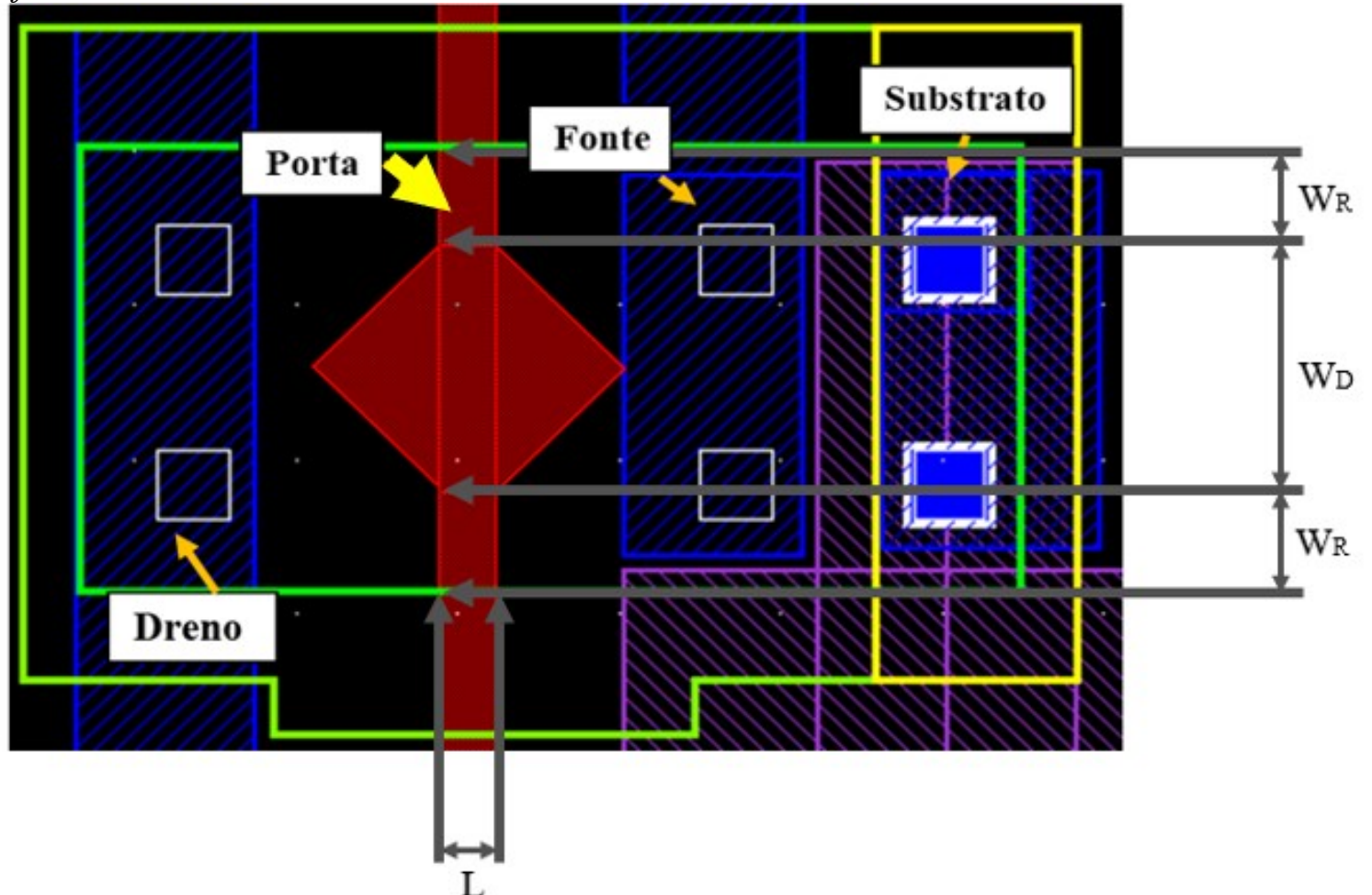

Fonte: Autor

Figura 27 - Leiaute do MOSFET do tipo Diamante Híbrido com ângulo $\alpha$ de $135^{\circ}$ feito no software ICStation.

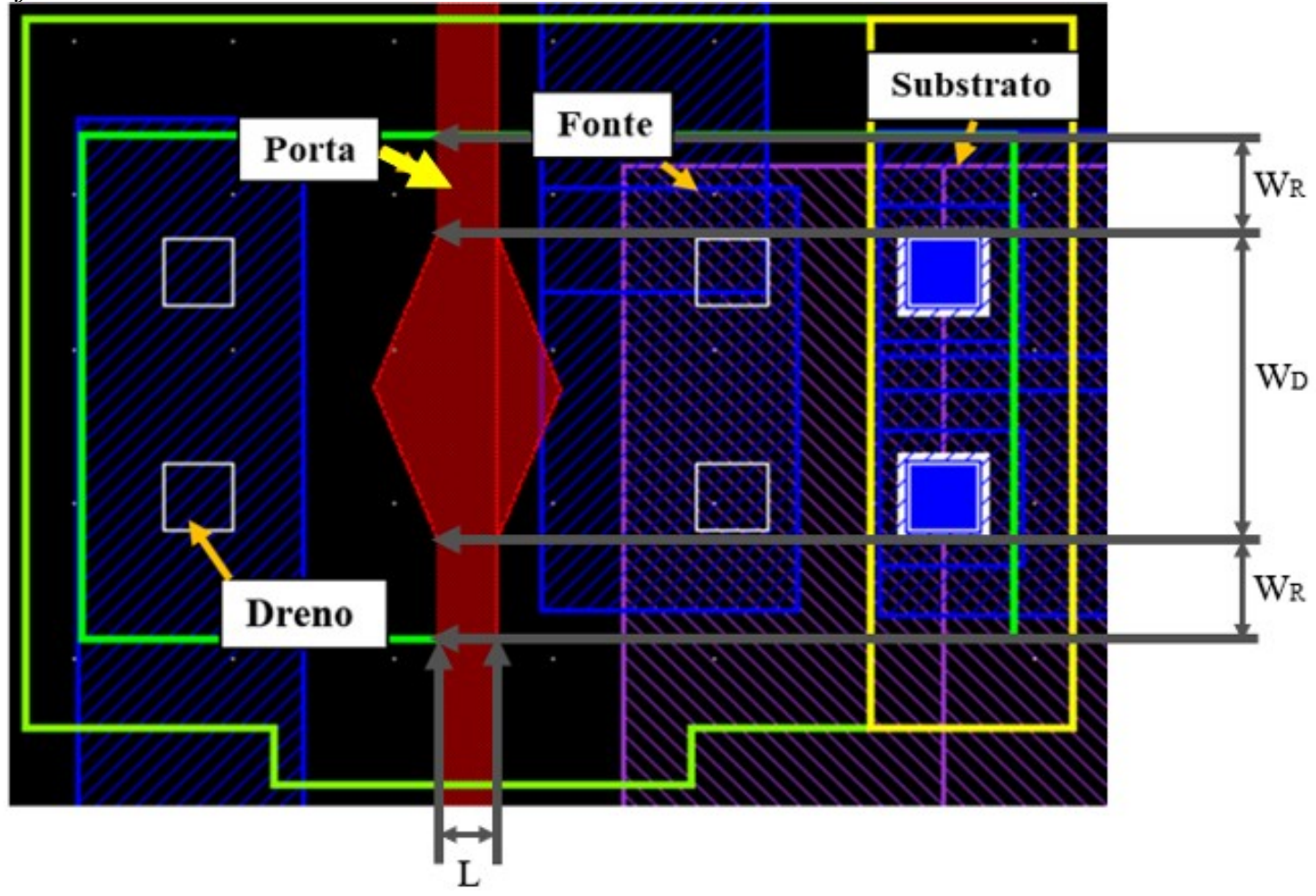

Fonte: Autor 
A Tabela 1 apresenta as dimensões dos MOSFETs Bulk que foram pesquisados neste projeto.

Tabela 1 - Tabela com os valores das dimensões dos MOSFETs estudados, no qual o valor de b é igual a $180 \mathrm{~nm}$ para todos os MOSFETs dos tipos Diamantes.

\begin{tabular}{c|c|c|c|c|c}
\hline MOSFET & $\mathbf{C M}$ & $\mathbf{C M}_{\mathbf{1}}$ & $\mathbf{D M ~}\left(\boldsymbol{\alpha}=\mathbf{4 5}^{\circ}\right)$ & $\mathbf{D M}(\boldsymbol{\alpha}=\mathbf{9 0})$ & $\mathbf{D M}\left(\boldsymbol{\alpha}=\mathbf{1 3 5}^{\circ}\right)$ \\
\hline $\mathrm{W}[\mu \mathrm{m}]$ & 0,42 & - & - & - & - \\
\hline $\mathrm{W}_{\mathrm{R}}[\mu \mathrm{m}]$ & - & 0,25 & - & - & - \\
\hline $\mathrm{W}_{\mathrm{D}}[\mu \mathrm{m}]$ & - & - & 1,60 & 1,44 & 1,60 \\
\hline $\mathrm{B}[\mu \mathrm{m}]$ & - & - & 2,57 & 0,98 & 0,58 \\
\hline $\mathrm{L}[\mu \mathrm{m}]$ & 0,18 & 0,18 & - & - & - \\
\hline $\mathrm{L}_{\mathrm{D}}[\mu \mathrm{m}]$ & - & - & 1,38 & 0,58 & 0,38 \\
\hline $\mathrm{L}_{\mathrm{eff}}[\mu \mathrm{m}]$ & - & - & 0,90 & 0,47 & 0,34 \\
\hline $\mathrm{A}_{\mathrm{G}}\left[\mu \mathrm{m}^{2}\right]$ & 0,08 & 0,05 & 2,21 & 0,83 & 0,61 \\
\hline$(\mathrm{W} / \mathrm{L})$ & 2,33 & 1,39 & - & - & - \\
\hline$(\mathrm{W} / \mathrm{L}) \mathrm{HDM}$ & - & - & 3,94 & 5,27 & 6,99 \\
\hline
\end{tabular}

Fonte: Autor

Como mencionado no capítulo anterior, o valor de (W/L)HDM será utilizado para realizar a normalização dos parâmetros elétricos e as figuras de mérito quando necessárias, a fim de eliminar os efeitos geométricos dos MOSFETs que estão sendo estudados.

\subsection{TENSÃO DE LIMIAR}

As curvas das IDS em função da $V_{G S}$ utilizadas para poder realizar a extração da $V_{\text {TH }}$ dos MOSFETs são apresentadas na Figura 28 e considerando-se uma tensão entre o dreno e a fonte igual a $50 \mathrm{mV}$. 
Figura 28 - Curvas das correntes entre o dreno e a fonte em função da tensão entre a porta e a fonte dos HDMs e o CM, para uma $\mathrm{V}_{\mathrm{DS}}$ igual a $50 \mathrm{mV}$.

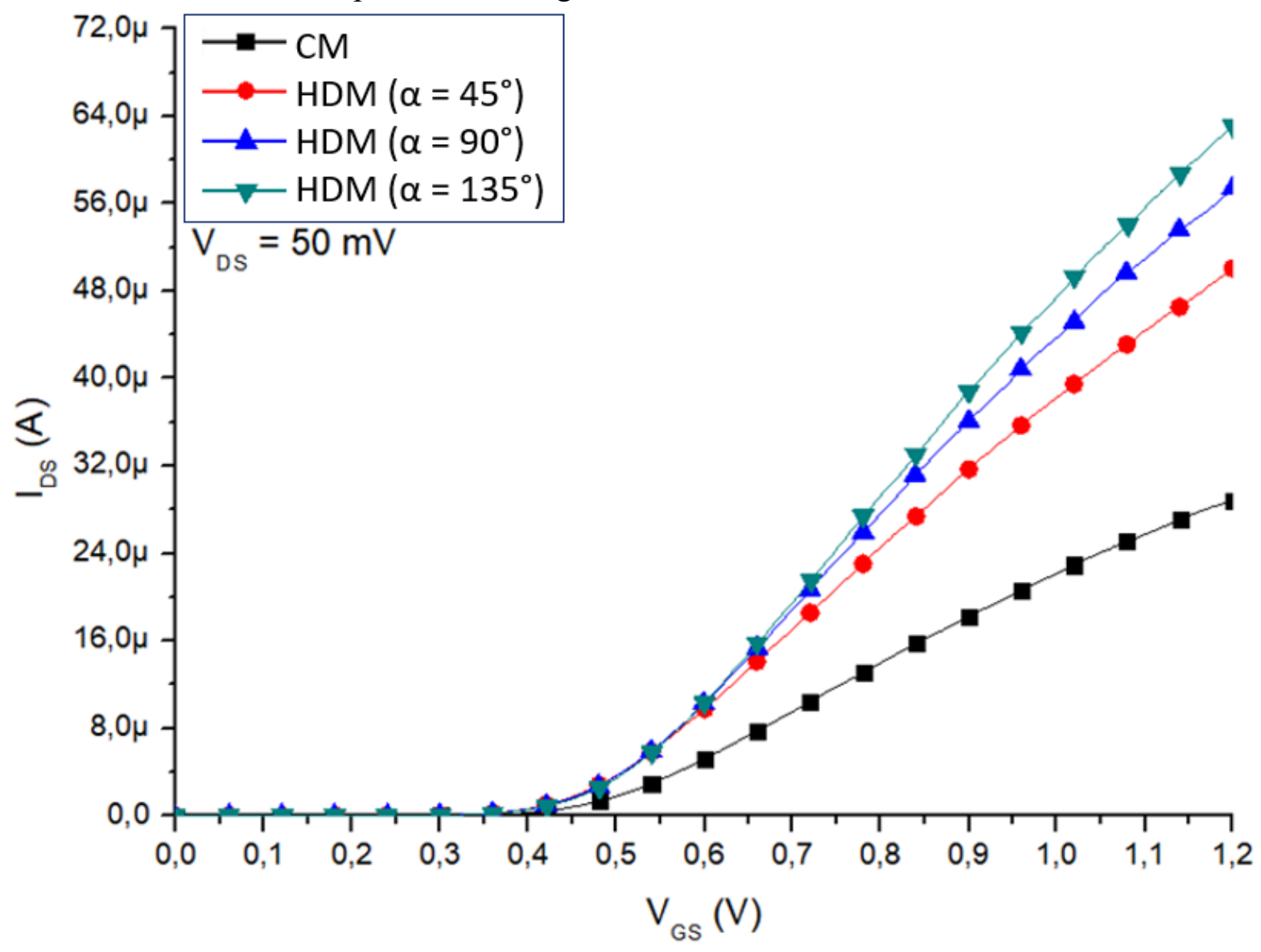

Fonte: Autor

A Tabela 2 apresenta os valores das tensões de limiar extraídas das curvas ilustradas na Figura 28, utilizando-se o método da segunda derivada de IDs em função de $V_{G S}$ para $V_{D S}$ igual a $50 \mathrm{mV}$.

Tabela 2 - Valores das tensões de limiar para os diferentes dispositivos, extraído com uma $\mathrm{V}_{\mathrm{DS}}$ igual a $50 \mathrm{mV}$.

\begin{tabular}{c|c}
\hline MOSFETs & $\mathrm{V}_{\mathrm{TH}}[\mathrm{V}]$ \\
\hline $\mathbf{C M}$ & 0,490 \\
\hline $\mathbf{H D M}\left(\boldsymbol{\alpha}=\mathbf{4 5} \mathbf{5}^{\circ}\right)$ & 0,485 \\
\hline $\mathbf{H D M}\left(\boldsymbol{\alpha}=\mathbf{9 0}^{\circ}\right)$ & 0,490 \\
\hline $\mathbf{H D M}\left(\boldsymbol{\alpha}=\mathbf{1 3 5}^{\circ}\right)$ & 0,490 \\
\hline
\end{tabular}

Fonte: Autor

As diferenças entre as $\mathrm{V}_{\mathrm{TH}}$ desses transistores estão dentro das especificações do processo de fabricação que é $0,45 \mathrm{~V} \pm 10 \%$, conforme definido pelo fabricante (TSMC). 


\subsection{CURVA [IDs/(W/L)] EM FUNÇÃO DE V $\mathrm{GT}$}

O gráfico da corrente entre o dreno e a fonte normalizada pela razão de aspecto dos MOSFETs em função da $V_{G T}$ para um determinado valor de $V_{D S}$, é uma das curvas mais importantes deste projeto de pesquisa, uma vez que é a base para a caracterização elétrica experimental da distorção harmônica total do seu sinal de saída, no qual devem operar na região de saturação para a aplicação de CIs CMOS analógicos.

É realizada a normalização da IDs pela razão de aspecto dos MOSFETs para poder eliminar as diferenças geométricas que existem entre eles e a curva é traçada em função da $V_{G T}$ para poder eliminar as diferenças de $\mathrm{V}_{\mathrm{TH}}$ deles.

A Figura 29 apresenta as curvas experimentais das IDs/(W/L) em função da $\mathrm{V}_{\mathrm{GT}}$ dos HDMs $\left(\alpha=45^{\circ} ; 90^{\circ} ; 135^{\circ}\right)$ e do $\mathrm{CM}$, para VDs igual a $700 \mathrm{mV}$.

Figura 29 - Curvas das correntes entre o dreno e a fonte normalizada em função da razão de aspecto em função da sobretensão de porta dos HDMs com diferentes ângulos $\alpha$ e do CM, para uma $V_{D S}$ igual a $700 \mathrm{mV}$ (Região de saturação).

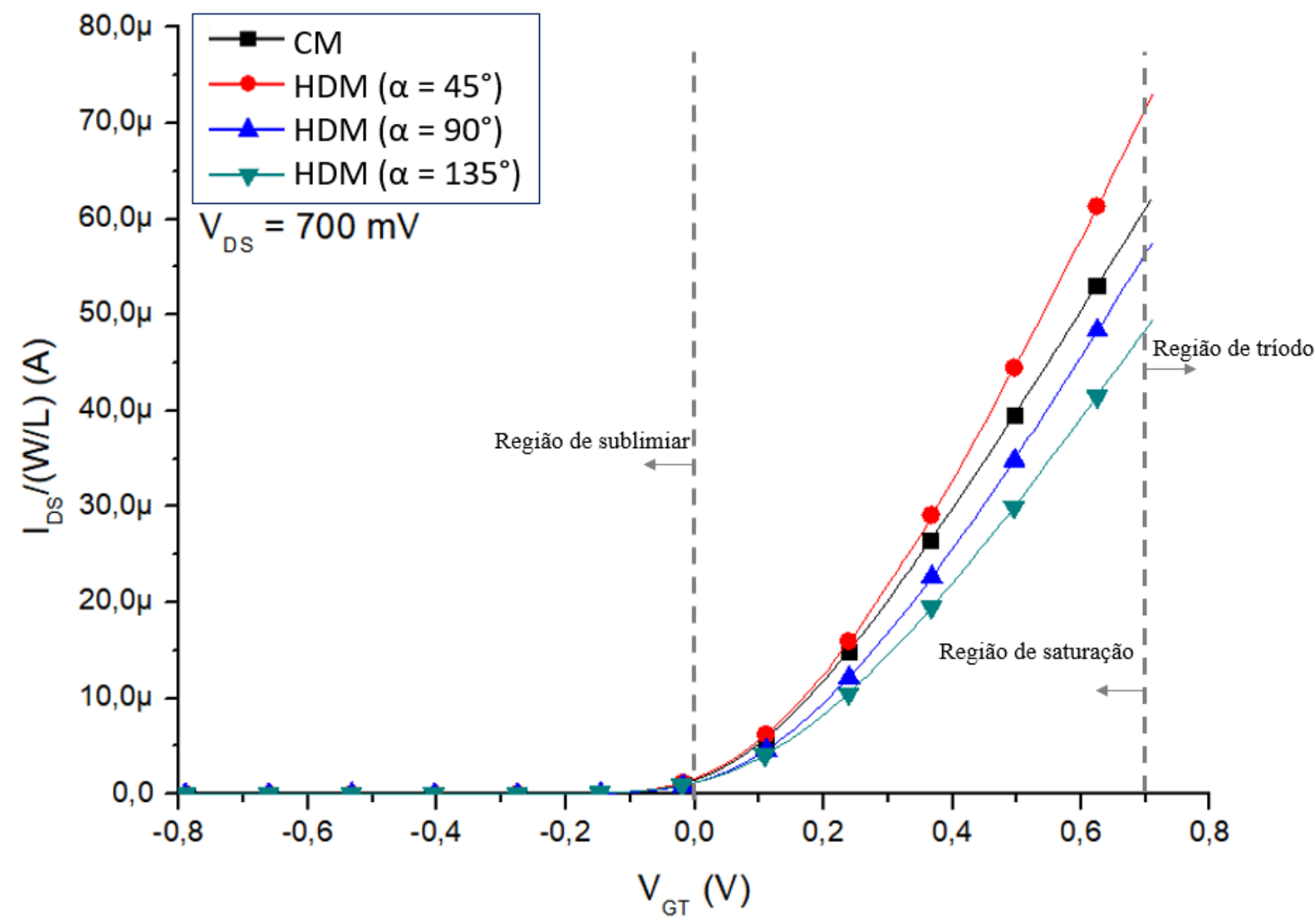

Fonte: Autor 
A Figura 29 mostra que o $\operatorname{HDM}\left(\alpha=45^{\circ}\right)$ possui uma IDS/(W/L) maior que a do CM equivalente (Diferença máxima de $17,65 \%)$ enquanto os demais HDMs $\left(\alpha=90^{\circ} ; 135^{\circ}\right)$ apresentam valores da IDS/(W/L) menores que a IDs/(W/L) do CM equivalente.

Pode-se justificar esses resultados através do LCE e o PAMDLE presentes no HDM ( $\alpha$ $=45^{\circ}$ ) os quais foram capazes de compensar a degradação da corrente entre o dreno e a fonte, apesar de possuir uma maior razão de aspecto $(3,94)$ em comparação ao CM equivalente $(2,33)$. Considerando-se um mesmo nível de corrente entre os dois MOSFETs [IDs/(W/L) $=15 \mu \mathrm{A}]$, o $\operatorname{HDM}\left(\alpha=45^{\circ}\right)$ e o $C M$, se tem a Equação (34).

$$
\left(\frac{I_{D S}}{W / L}\right)_{H D M\left(\alpha=45^{\circ}\right)}=\left(\frac{I_{D S}}{W / L}\right)_{C M}
$$

Ao substituir na Equação (34) a W/L correspondente de cada um dos MOSFET a parte referente ao $\mathrm{CM}$ teria um nível de $\operatorname{IDS} /(\mathrm{W} / \mathrm{L})$ maior que à do $\operatorname{HDM}\left(\alpha=45^{\circ}\right)$, todavia isso não ocorre, porque apesar do CM ter uma W/L menor que à do HDM $\left(\alpha=45^{\circ}\right)$, esse conta com o DM em sua estrutura o qual é capaz de aumentar a IDs devido ao LCE e PAMDLE presentes [13].

Já os HDMs com $\alpha$ iguais a $90^{\circ}$ e $135^{\circ}$ apesar de terem presentes em sua estrutura o LCE e o PAMDLE, que são responsáveis por aumentar a IDS no dispositivo, eles não foram capazes de compensar as suas maiores $\mathrm{W} / \mathrm{L}$.

\subsection{CURVA $\left[\mathrm{R}_{\mathrm{DS}} *(\mathrm{~W} / \mathrm{L})\right]$ EM FUNÇÃO DE V $\mathrm{GT}$}

A curva da $\mathrm{R}_{\mathrm{DS}} *(\mathrm{~W} / \mathrm{L})$ em função da $\mathrm{V}_{\mathrm{GT}}$, apresentada na Figura 30, é utilizada para poder realizar a extração da $\mathrm{R}_{\mathrm{S}}$ normalizada pela razão de aspecto dos MOSFETs [RS*(W/L)] e considerando uma $\mathrm{V}_{\mathrm{DS}}$ igual a $50 \mathrm{mV}$. 
Figura 30 - Curvas das resistências entre o dreno e a fonte normalizadas pela razão de aspecto dos HDMs e o CM em função de $\mathrm{V}_{\mathrm{GT}}$, para uma $\mathrm{V}_{\mathrm{DS}}$ igual a $50 \mathrm{mV}$.

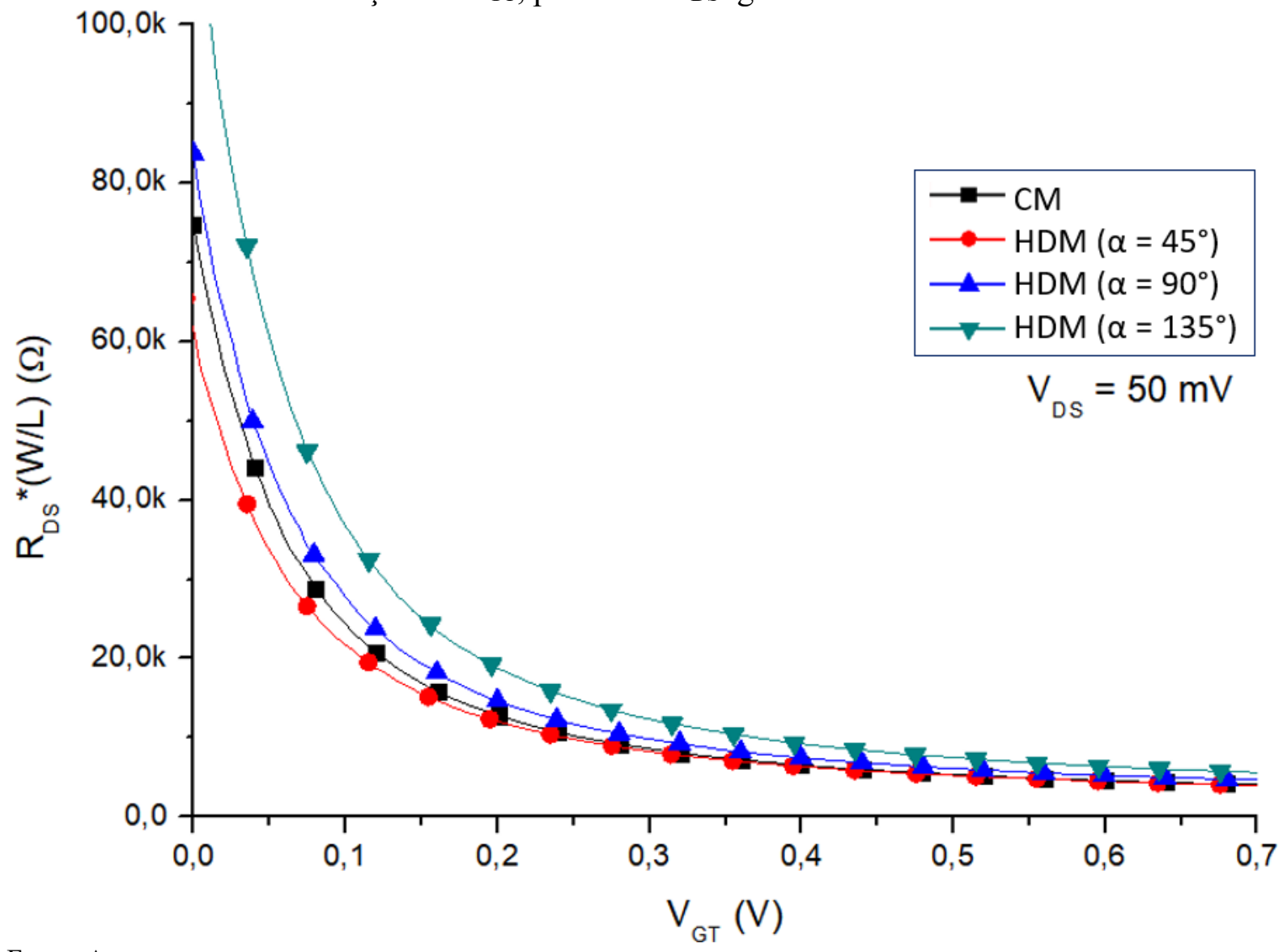

Fonte: Autor

Como o valor de $\mathrm{R}_{D S} *(\mathrm{~W} / \mathrm{L})$ foi obtido para uma $\mathrm{V}_{D S}$ baixa $\left(\mathrm{V}_{D S}=50 \mathrm{mV}\right)$ e para um valor de $\mathrm{V}_{\mathrm{GT}}$ alto $\left(\mathrm{V}_{\mathrm{GT}}=0,7 \mathrm{~V}\right)$, o valor de $\mathrm{R}_{\mathrm{CH}}$, conforme a Equação (7), cai drasticamente, pois a interface entre o canal e o óxido de porta apresenta-se totalmente invertida, fazendo com que a $\mathrm{R}_{\mathrm{DS}} *(\mathrm{~W} / \mathrm{L})$ se torne praticamente igual a $\mathrm{RS}_{\mathrm{S}}(\mathrm{W} / \mathrm{L})$ [1][3]. Dessa maneira têm-se os valores de $\mathrm{R}_{\mathrm{S}}$ normalizados por W/L.

Analisando as curvas da Figura 30, para um valor de VGT igual a 0,7 V o HDM ( $\alpha=$ $45^{\circ}$ ) possui uma $\mathrm{RS}_{\mathrm{S}}(\mathrm{W} / \mathrm{L})$ igual a $3,98 \mathrm{k} \Omega$, o $\mathrm{CM}$ possui uma $\mathrm{RS}_{\mathrm{S}} *(\mathrm{~W} / \mathrm{L})$ igual a $4,07 \mathrm{k} \Omega$, o $\operatorname{HDM}\left(\alpha=90^{\circ}\right)$ possui uma $\mathrm{RS}_{S}^{*}(\mathrm{~W} / \mathrm{L})$ igual a $4,65 \mathrm{k} \Omega$ e o $\operatorname{HDM}\left(\alpha=135^{\circ}\right)$ possui uma $\mathrm{Rs}_{\mathrm{S}} *(\mathrm{~W} / \mathrm{L})$ igual a $5,60 \mathrm{k} \Omega$.

Isso é justificado pelo LCE e o PAMDLE presentes nos HDMs. O LCE e o PAMDLE são mais pronunciados no $\operatorname{HDM}\left(\alpha=45^{\circ}\right)$, o que resulta em maior $\operatorname{IDS} /(\mathrm{W} / \mathrm{L})$ quando comparado aos demais MOSFETs estudados nesse projeto de pesquisa para uma mesma VDS num determinado $\mathrm{V}_{\mathrm{GT}}\left(\mathrm{V}_{\mathrm{DS}}\right.$ igual a $700 \mathrm{mV}$ e $\mathrm{V}_{\mathrm{GT}}$ igual $700 \mathrm{mV}$ ), e, consequentemente, obtémse menor resistência série, pela menor razão de aspecto do $\operatorname{HDM}\left(\alpha=45^{\circ}\right)$ em relação aos 
outros HDMs $\left(\alpha=90^{\circ} ; \alpha=135^{\circ}\right)$. Enquanto nos demais HDMs $\left(\alpha=90^{\circ} ; \alpha=135^{\circ}\right)$, mesmo tendo o LCE e o PAMDLE em sua estrutura devido a mudança de leiaute, apresentaram menor $\mathrm{IDS} /(\mathrm{W} / \mathrm{L})$ quando comparados ao $\mathrm{CM}$, considerando as mesma condições impostas no HDM $\left(\alpha=45^{\circ}\right)$, pois não tiveram seus efeitos evidenciados devido às suas maiores razões de aspecto, dessa maneira resulta numa maior Rs*(W/L) [3][13][29].

\subsection{TRANSCONDUTÂNCIA MÁXIMA DO MOSFET}

A Figura 31 ilustra as curvas das transcondutâncias normalizadas pelas razões de aspecto $\left[\mathrm{gm}_{\mathrm{m}} /(\mathrm{W} / \mathrm{L})\right]$ em função da $\mathrm{V}_{\mathrm{GT}}$ dos MOSFETs para uma polarização entre o dreno e a fonte igual a $700 \mathrm{mV}$ [Figura 30.(a)] e as curvas das $\mathrm{g}_{\mathrm{m}} /(\mathrm{W} / \mathrm{L})$ em função da $\mathrm{IDS}_{\mathrm{DS}} /(\mathrm{W} / \mathrm{L})$ para um $\mathrm{V}_{\text {DS }}$ igual a $700 \mathrm{mV}$ [Figura 30.(b)]. 
Figura 31 - Curvas das transcondutâncias normalizadas pelas razões de aspecto dos HDMs e do CM em função da $V_{\text {GT }}$ (a) e em função de $\operatorname{IDS} /(\mathrm{W} / \mathrm{L})$ (b), considerando uma tensão entre o dreno e a fonte igual a $700 \mathrm{mV}$.
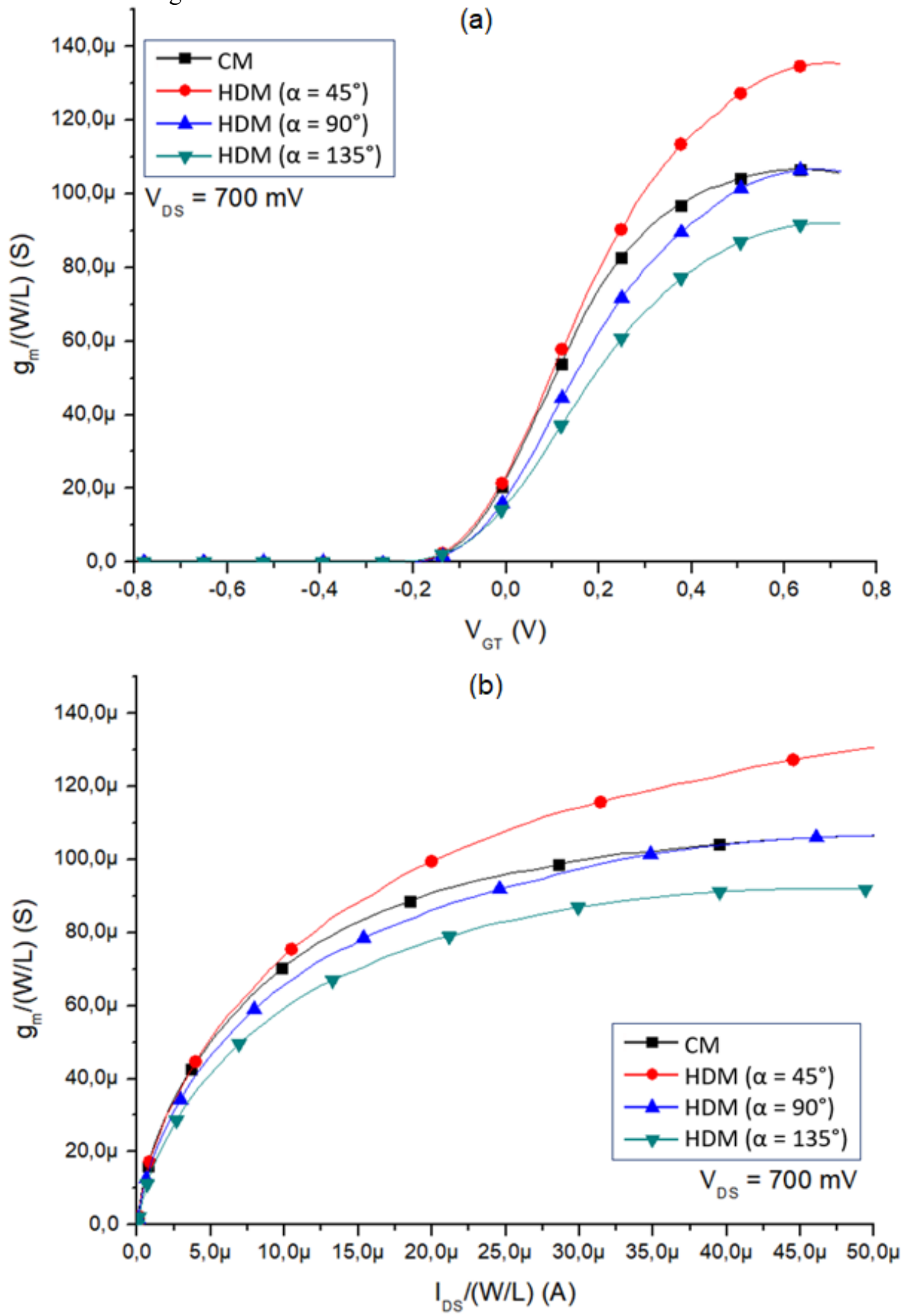

Fonte: Autor 
Baseando-se nas curvas da Figura 31.(a) obteve-se os valores das transcondutâncias dos MOSFETs (HDMs e CM) para um determinado ponto da tensão de polarização ( $V_{\mathrm{DS}}=700$ $\mathrm{mV}$ ), conforme descrito na Tabela 3.

Tabela 3 - Valores das transcondutâncias máximas dos MOSFETs com leiautes de porta dos tipos Diamante Híbrido e retangular para uma $V_{D S}$ igual a $700 \mathrm{mV}$.

\begin{tabular}{c|c|c}
\hline MOSFETs & $\mathbf{g}_{\mathbf{m}} /(\mathbf{W} / \mathbf{L})[\boldsymbol{\mu S}]$ & $\begin{array}{c}\text { Desvio entre os } \mathbf{g}_{\mathbf{m}} /(\mathbf{W} / \mathbf{L}) \mathbf{d o s} \\
\text { HDMs em relação ao CM [\%] }\end{array}$ \\
\hline $\mathbf{C M}$ & $105,89\left(\mathrm{~V}_{\mathrm{GT}}=0,700 \mathrm{~V}\right)$ & - \\
\hline HDM $\left(\boldsymbol{\alpha}=\mathbf{4 5}^{\circ}\right)$ & $135,52\left(\mathrm{~V}_{\mathrm{GT}}=0,700 \mathrm{~V}\right)$ & $+27,98 \%$ \\
\hline HDM $\left(\boldsymbol{\alpha}=\mathbf{9 0}^{\circ}\right)$ & $106,05\left(\mathrm{~V}_{\mathrm{GT}}=0,700 \mathrm{~V}\right)$ & $+0,15 \%$ \\
\hline HDM $\left(\boldsymbol{\alpha}=\mathbf{1 3 5}^{\circ}\right)$ & $92,01\left(\mathrm{~V}_{\mathrm{GT}}=0,700 \mathrm{~V}\right)$ & $-13,11 \%$ \\
\hline
\end{tabular}

Fonte: Autor

Analisando a Tabela 3 nota-se que, para um valor de $\mathrm{V}_{\text {GT }}$ igual a $700 \mathrm{mV}$ e $\mathrm{V}_{\text {DS }}$ igual a $700 \mathrm{mV}$, a $\mathrm{g}_{\mathrm{m}} /(\mathrm{W} / \mathrm{L})$ do $\mathrm{HDM}\left(\alpha=45^{\circ}\right)$ é $27,98 \%$ maior que a do CM equivalente, o HDM ( $\alpha$ $\left.=90^{\circ}\right)$ tem uma $g_{m} /(\mathrm{W} / \mathrm{L})$ praticamente igual à do $\mathrm{CM}$, e já para a $\mathrm{g}_{\mathrm{m}} /(\mathrm{W} / \mathrm{L})$ do $\operatorname{HDM}\left(\alpha=135^{\circ}\right)$ teve um valor cerca de $13,11 \%$ menor que a $\mathrm{g}_{\mathrm{m}} /(\mathrm{W} / \mathrm{L})$ do $\mathrm{CM}$.

Isso justifica-se porque o $\operatorname{HDM}\left(\alpha=45^{\circ}\right)$ apresentou o LCE e o PAMDLE, presentes nesse inovador estilo de leiaute para os MOSFETs, capazes de compensar sua maior razão de aspecto em relação ao $\mathrm{CM}$.

Portanto a substituição dos CMs por HDMs equivalentes com $\alpha$ igual a $45^{\circ}$ é viável para aumentar a transcondutância dos MOSFETs.

\subsection{INCLINAÇÕES DE SUBLIMIAR DOS MOSFETS COM LEIAUTES DE PORTA TIPO DIAMANTE HÍBRIDO E DO TIPO RETANGULAR}

A inclinação de sublimiar (SS) é o parâmetro que representa o quão rápido um MOSFET passa do estado desligado para o estado ligado, o qual é obtido por meio da Equação (35) [1].

$$
S S=\frac{d V_{G S}}{d\left(\log I_{D S}\right)}=\frac{n * k * T}{q} * \ln (10)
$$

A Figura 32 apresenta as curvas da inclinação de sublimiar dos MOSFETs dos tipos Diamante Híbrido e retangular em função da sobretensão de porta, para VDS igual a $700 \mathrm{mV}$. 
Figura 32 - Gráfico de IDS/(W/L) em função de VGT na escala logarítmica dos MOSFETs com leiautes de porta dos tipos Diamante Híbrido e retangular, para uma $\mathrm{V}_{\mathrm{DS}}$ igual a $700 \mathrm{mV}$.

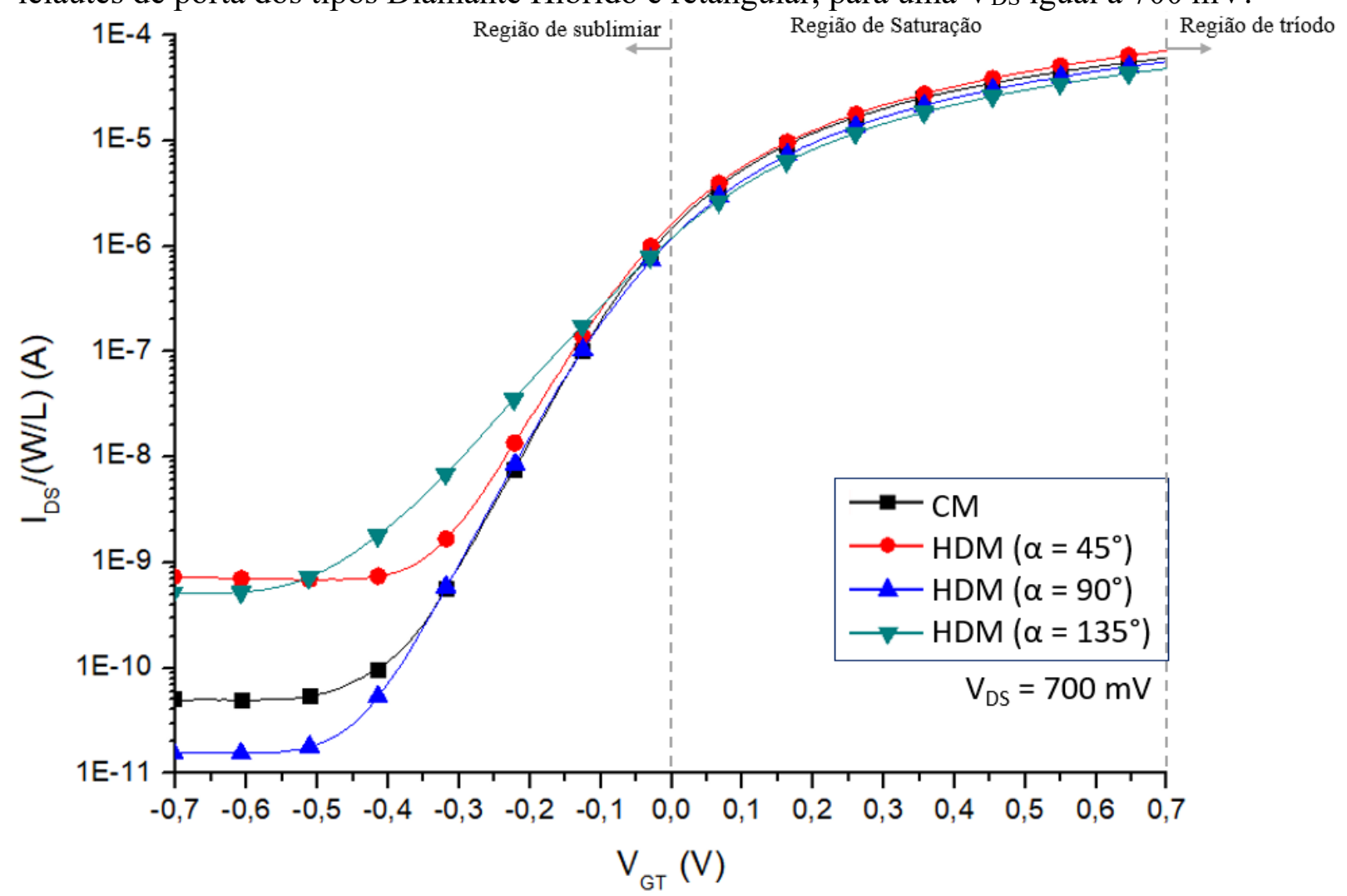

Fonte: Autor

A inclinação de sublimiar, a corrente entre o dreno e a fonte de estado ligado (Ion) normalizado pela razão de aspecto [IoN/(W/L)], a corrente entre o dreno e a fonte de estado desligado (IofF) normalizado pela razão de aspecto [IofF/(W/L)] e a razão Ion/Ioff dos MOSFETs considerados nesta dissertação são apresentados na Tabela 4.

Tabela 4 - Valores de SS, Ion, Ioff e Ion/Ioff dos MOSFETs com leiautes dos tipos Diamante Híbrido e retangular, considerando-se uma VDS igual a $700 \mathrm{mV}$.

\begin{tabular}{c|c|c|c|c}
\hline MOSFETs & $\mathbf{C M}$ & HDM $\left(\boldsymbol{\alpha}=\mathbf{4 5}^{\mathbf{}}\right)$ & HDM $\left(\boldsymbol{\alpha}=\mathbf{9 0}^{\mathbf{}}\right)$ & HDM $\left(\boldsymbol{\alpha}=\mathbf{1 3 5}^{\mathbf{}}\right)$ \\
\hline SS [mV/década] & 85,68 & 95,18 & 82,25 & 134,54 \\
\hline $\mathrm{I}_{\mathrm{ON}} /(\mathrm{W} / \mathrm{L})[\mu \mathrm{A}]$ & 60,92 & 71,43 & 56,42 & 48,39 \\
\hline $\mathrm{I}_{\mathrm{OFF}} /(\mathrm{W} / \mathrm{L})[\mathrm{pA}]$ & 49,98 & 712,29 & 15,63 & 508,67 \\
\hline $\begin{array}{c}\mathrm{I}_{\mathrm{ON}} / \mathrm{I}_{\mathrm{OFF}}[\mathrm{Ordem} \mathrm{de} \\
\text { grandeza }(\mathrm{og})]\end{array}$ & 6 & 5 & 6 & 4 \\
\hline
\end{tabular}

Fonte: Autor

Na Tabela 4, as SS do $\mathrm{CM}$ e do $\operatorname{HDM}\left(\alpha=90^{\circ}\right)$ são praticamente idênticas, como visto na Figura 32, com uma variação entre si de 4\%, visto que foram manufaturados com a mesma 
tecnologia de CI CMOS. O HDM $\left(\alpha=45^{\circ}\right)$ e o $\operatorname{HDM}\left(\alpha=135^{\circ}\right)$ possuíram valores de SS cerca de $11,09 \%$ e $57,03 \%$, respectivamente. Isso é justificado devido à degradação da corrente de estado desligado, que foi muito elevada em ambos os MOSFETs.

Ao prosseguir com as análises da Tabela 4 nota-se que a Ioff/(W/L) (Considerando uma $\mathrm{V}_{\mathrm{GT}}$ igual a $-0,6 \mathrm{~V}$ ) dos HDMs com ângulos $\alpha$ iguais a $45^{\circ}$ e a $135^{\circ}$ foram maiores que o valor da $\operatorname{Ioff} /(\mathrm{W} / \mathrm{L})$ do CM equivalente, cerca de 14,35 vezes menor e 10,18 vezes menor, respectivamente. Isso ocorre porque os $\operatorname{HDMs}\left(\alpha=45^{\circ} ; 135^{\circ}\right)$, apesar de possuírem maiores LeffS em comparação ao CM equivalente, apresentaram um perímetro da junção p-n maior que o do CM equivalente, e seus LEFs não foram capazes de se sobrepor a esse efeito de perímetro, dessa forma, suas correntes de fuga foram maiores que a do CM equivalente [13][29]. Como consequência disso, suas razões de Ion/Ioff foram 1 og menor e 2 og menor, respectivamente, em relação ao Ion/Ioff do CM equivalente.

Já o HDM $\left(\alpha=90^{\circ}\right)$ apresentou uma IofF/(W/L) menor, cerca de $68,7 \%$ menor que a do $\mathrm{CM}$ equivalente. Isso ocorre porque, apesar de apresentar um perímetro da junção p-n maior que a do CM equivalente, assim como nos casos dos HDMs com ângulo $\alpha$ iguais a $45^{\circ}$ e a $135^{\circ}$, esse não sofreu degradação do seu campo elétrico longitudinal, que é o responsável por aumentar o comprimento da região de depleção do dispositivo [8][33]. Assim, pode-se concluir que o HDM $\left(\alpha=90^{\circ}\right)$ apresentou o melhor compromisso entre o LEF e o perímetro da junção p-n por esse apresentar menor Ioff/(W/L) em relação ao valor de IofF/(W/L) do CM equivalente.

Também é possível observar que a Ion/(W/L) do HDM $\left(\alpha=45^{\circ}\right)$ possui um valor cerca de $17,25 \%$ maior que à do $\mathrm{CM}$ equivalente, considerando uma $\mathrm{V}_{\mathrm{GT}}$ igual a $0,7 \mathrm{~V}$. Adicionalmente nota-se que o valor da Ion/(W/L) do HDM $\left(\alpha=90^{\circ}\right)$ possui um valor 7,39\% menor do que o observado no CM equivalente, para uma $\mathrm{V}_{\mathrm{GT}}$ igual a $0,7 \mathrm{~V}$. E para o HDM ( $\alpha$ $=135^{\circ}$ ) constatou-se uma Ion/(W/L) 20,57\% menor que a do CM equivalente, para uma $\mathrm{V}_{\mathrm{GT}}$ igual a $0,6 \mathrm{~V}$. Isso é justificado porque os HDMs com ângulos iguais a $90^{\circ}$ e $135^{\circ}$ não apresentam LCE e PAMDLE capazes de compensar os seus maiores Leff em relação ao do CM equivalente e o HDM com ângulo $\alpha$ igual a $45^{\circ}$ apresentou um valor de IoN/(W/L) praticamente igual ao valor da IoN/(W/L) do CM equivalente. 


\subsection{CURVA DE gm/IDs EM FUNÇÃO DE IDS/(W/L) PARA OS MOSFETS COM LEIAUTES DE PORTA DOS TIPOS DIAMANTE HÍBRIDO E RETANGULAR}

Uma figura de mérito muito importante para o desenvolvimento de projetos de CIs CMOS de baixa potência e baixa tensão analógicos é a curva da razão $g_{m} / I_{D S}$ em função de IDS/(W/L). Essa curva é também utilizada para determinar o regime de inversão de canal dos MOSFETs [20][35]. Essa curva é obtida por meio da expressão apresentada na Equação (36) [20].

$$
\frac{g_{m}}{I_{D S}}=\frac{1}{I_{D S}} \frac{\partial I_{D S}}{\partial V_{G S}}=\frac{\partial\left(\ln I_{D S}\right)}{\partial V_{G S}}=\frac{\partial\left\{\ln \left[\frac{I_{D S}}{\left(\frac{W}{L}\right)}\right]\right\}}{\partial V_{G S}}
$$

A Figura 33 ilustra as curvas das razões de $\mathrm{g}_{\mathrm{m}} / \mathrm{IDs}$ em função da corrente entre o dreno e a fonte normalizada em função da razão de aspecto dos MOSFETs dos tipos Diamante Híbrido e retangular, para a condição de polarização de tensão entre o dreno e a fonte igual a $700 \mathrm{mV}$. Nas curvas apresentadas na Figura 33, o limiar entre a inversão fraca e a moderada foi a partir

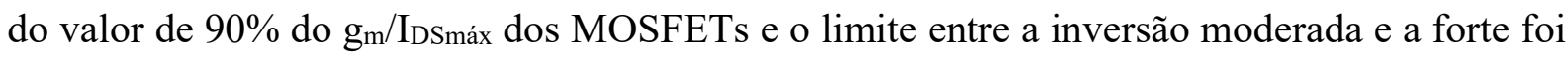
a partir do valor de $10 \%$ do $g_{m} / I_{D S m a ́ x}$ dos MOSFETs [20][35]. 
Figura 33 - Curvas experimentais das razões de $\mathrm{g}_{\mathrm{m}} / \mathrm{IDS}$ em função da corrente entre o dreno e a fonte normalizada em função da razão de aspecto [IDS/(W/L)] dos MOSFETs com leiautes de porta dos tipos Diamante Híbrido e retangular.

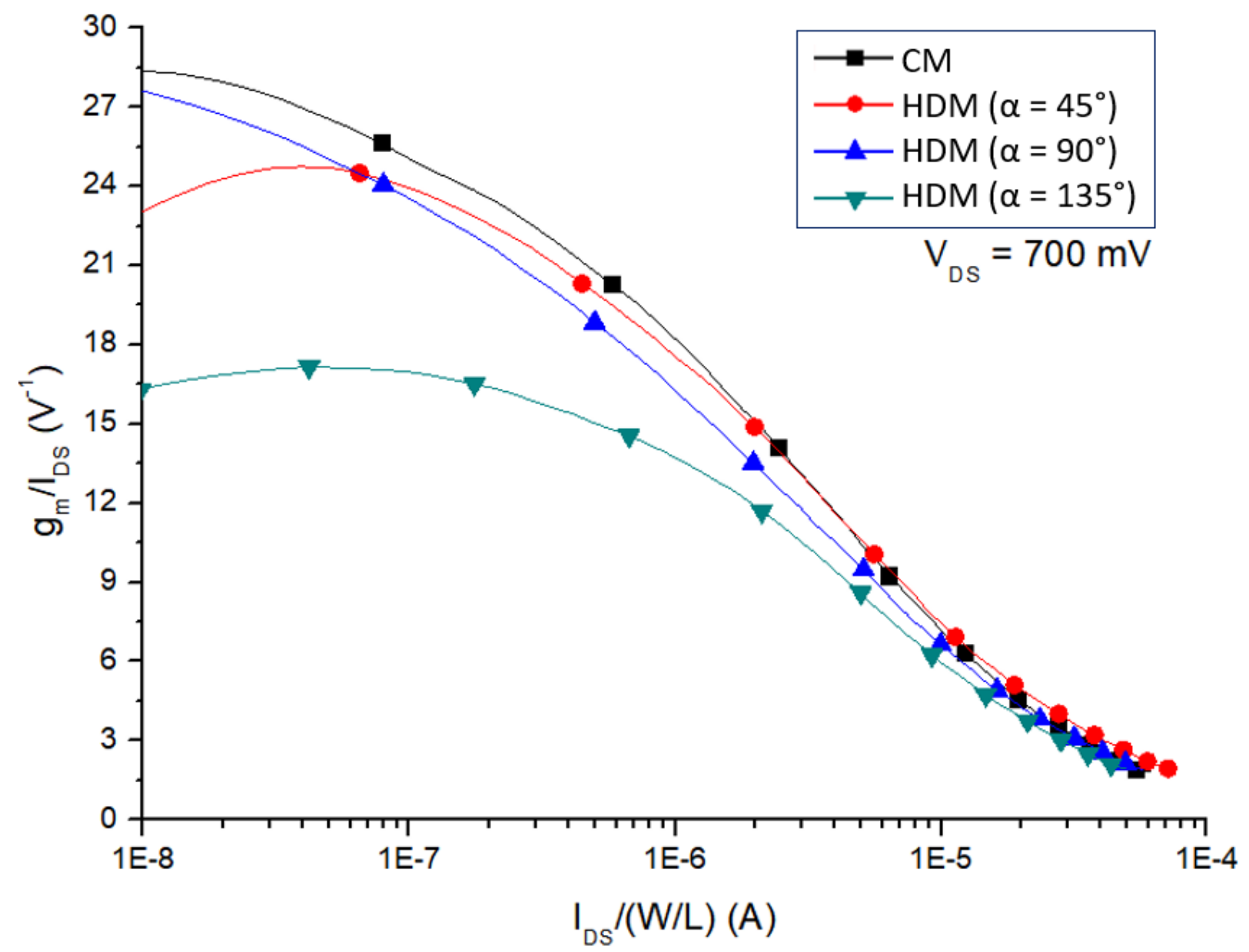

Fonte: Autor

Analisando-se a curva da Figura 33 observa-se que $g_{m} / I_{D s}$ do HDM com ângulo $\alpha$ igual a $135^{\circ}$ está bastante degradado em relação aos demais dispositivos, apresenta um valor de $\mathrm{g}_{\mathrm{m}} / \mathrm{IDs}$ cerca de $43 \%\left[\mathrm{IDS} /(\mathrm{W} / \mathrm{L})=1 \cdot 10^{-8} \mathrm{~A}\right]$ menor que o do $\mathrm{CM}$. Isso pode ser justificado por ter apresentado uma SS muito elevada (1,57 vezes maior) em relação à do CM equivalente, considerando um VDs igual a $700 \mathrm{mV}$ [1][35].

Além dessa figura de mérito apresentado na Figura 33, há outra a ser considerada, Figura 34, que é representada pela $\mathrm{g}_{\mathrm{m}} / \mathrm{I}_{\mathrm{DS}}$ em função da $\mathrm{V}_{\mathrm{GT}}$. Essa curva é utilizada para analisar como se comporta a distorção harmônica dos MOSFETs em função do regime de inversão de canal dos MOSFETs, que na figura apresentada ilustra a região de inversão de canal moderada e a forte. 
Figura 34 - Curvas experimentais das razões de $g_{m} / \mathrm{IDs}$ em função da sobretensão de porta dos MOSFETs com leiautes de porta dos tipos Diamante Híbrido e retangular.

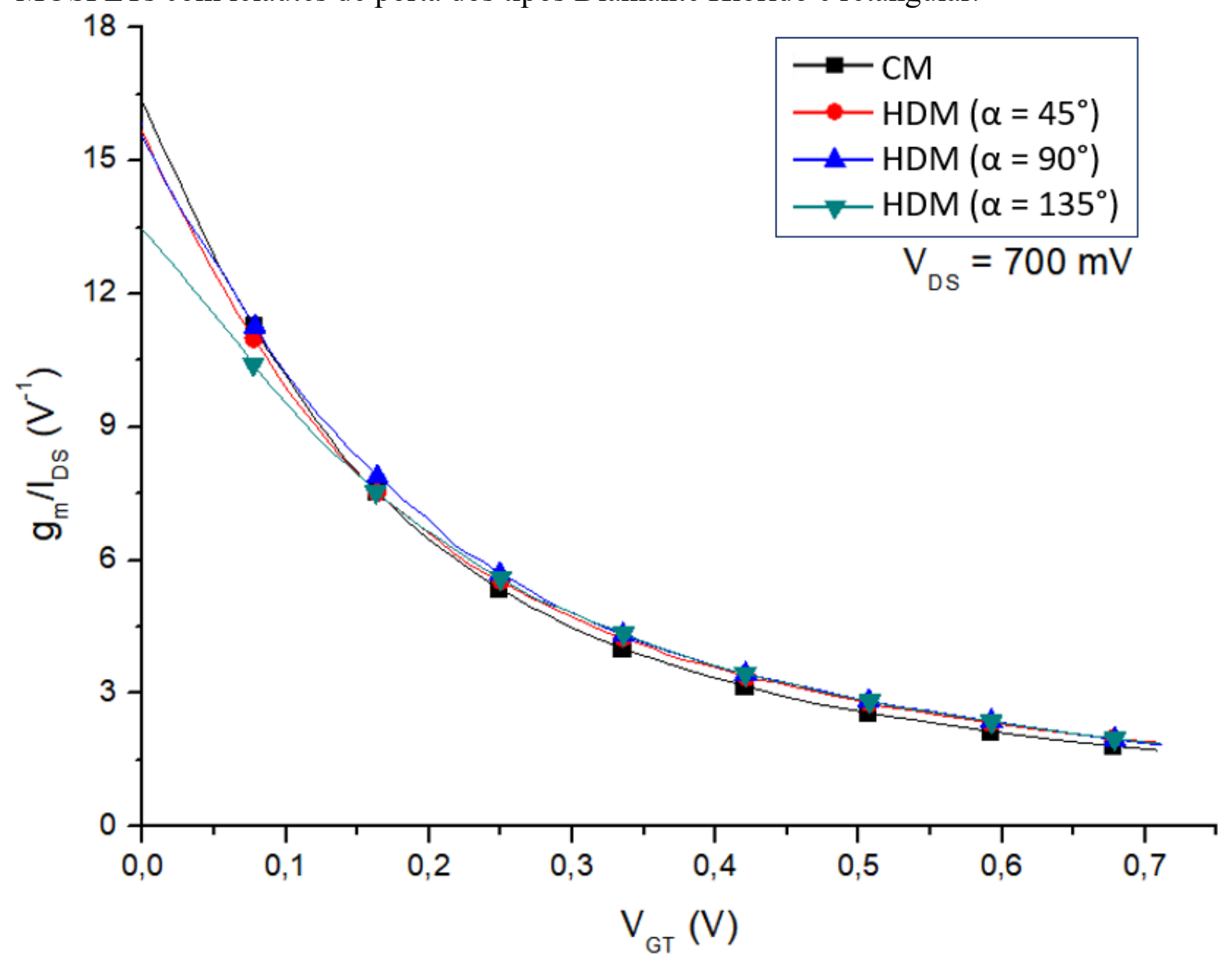

Fonte: Autor

Analisando as curvas da Figura 34, os HDMs com $\alpha$ iguais a $90^{\circ}$ e $45^{\circ}$ apresentaram valores de $\mathrm{g}_{\mathrm{m}} / \mathrm{I}_{\mathrm{DS}}$ aproximadamente $4,6 \%$ menor em relação ao valor do $\mathrm{CM}$ equivalente, considerando-se um $\mathrm{V}_{\mathrm{GT}}$ igual a $0 \mathrm{~V}$. Verifica-se também que o HDM com $\alpha$ igual a $135^{\circ}$ apresenta um valor da $\mathrm{g}_{\mathrm{m}} / \mathrm{IDS} 17,4 \%$ menor que o do CM equivalente, considerando um $\mathrm{V}_{\mathrm{GT}}$ nulo.

\subsection{CURVA IDS/(W/L) EM FUNÇÃO DE VDs E CONDUTÂNCIA DE DRENO}

A fim de obter os valores da $\mathrm{V}_{\mathrm{EA}}$ e do Av dos MOSFETs, é necessária a obtenção das curvas da IDS/(W/L) em função da VDS desses dispositivos.

As curvas das IDS/(W/L) em função de VDs são ilustradas na Figura 35 e considerandose uma $V_{\text {GT }}$ igual a $700 \mathrm{mV}$. 
Figura 35 - Curvas experimentais das IDS/(W/L) em função da $V_{D S}$, considerando-se uma $V_{G T}$ igual $700 \mathrm{mV}$, dos MOSFETs com leiautes de porta dos tipos Diamante Híbrido e retangular.

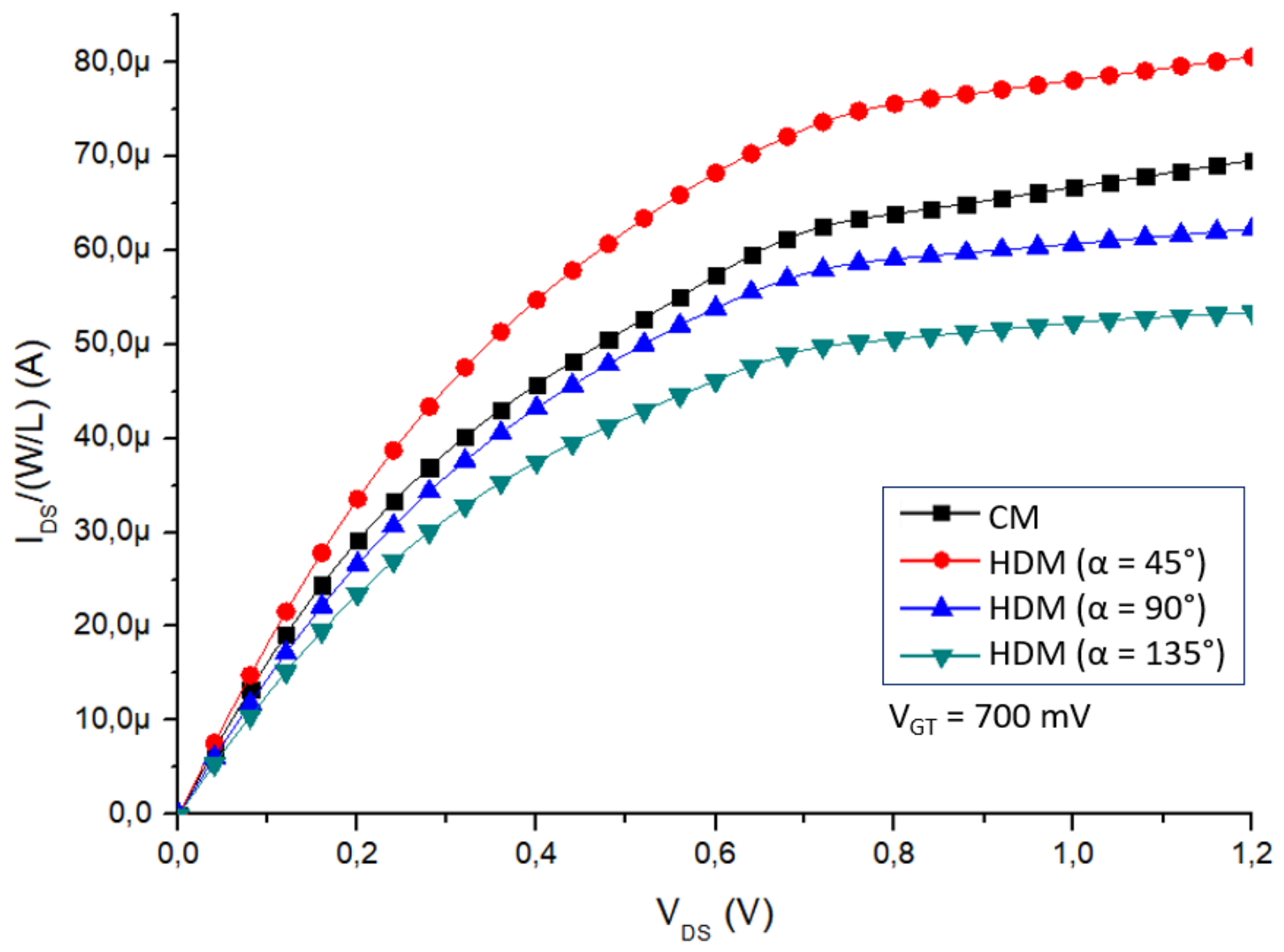

Fonte: Autor

Analisando-se a Figura 35, verifica-se que o $\operatorname{HDM}\left(\alpha=45^{\circ}\right)$ apresentou uma IDS/(W/L) na região de saturação maior que a do CM equivalente (Aproximadamente 17,07\%, para uma $\mathrm{V}_{\mathrm{DS}}$ igual a 1,0 V) quando polarizado com uma $\mathrm{V}_{\mathrm{GT}}$ igual a $700 \mathrm{mV}$. Esse maior valor de $\mathrm{IDS} /(\mathrm{W} / \mathrm{L})$ do HDM com $\alpha$ igual a $45^{\circ}$ em relação ao CM equivalente é justificado pelos efeitos de LCE e PAMDLE presentes em sua estrutura. Já para o HDM com $\alpha$ igual a $90^{\circ}$ e com $\alpha$ igual a $135^{\circ}$, apesar de possuírem esses mesmos efeitos em suas estruturas, não são capazes de compensar os seus valores de IDS/(W/L) em virtude de seus maiores valores de W/L.

Derivando a curva da IDS/(W/L) em função de VDS obtêm-se os valores da condutância de saída normalizados pela razão de aspecto [ $\left.\mathrm{g}_{\mathrm{D}} /(\mathrm{W} / \mathrm{L})\right]$ dos MOSFETs em função de $\mathrm{V}_{\mathrm{DS}}$. As curvas das $\mathrm{g}_{\mathrm{D}} /(\mathrm{W} / \mathrm{L})$ em função dos MOSFETs estudados são exibidas na Figura 36, considerando-se uma $\mathrm{V}_{\mathrm{GT}}$ igual a $700 \mathrm{mV}$. 
Figura 36 - Curvas experimentais das condutâncias de saída normalizadas pela razão de aspecto $\left[\mathrm{gD}_{\mathrm{D}} /(\mathrm{W} / \mathrm{L})\right]$ em função da tensão entre o dreno e a fonte dos MOSFETs com leiautes de porta dos tipos Diamante Híbrido e o retangular.

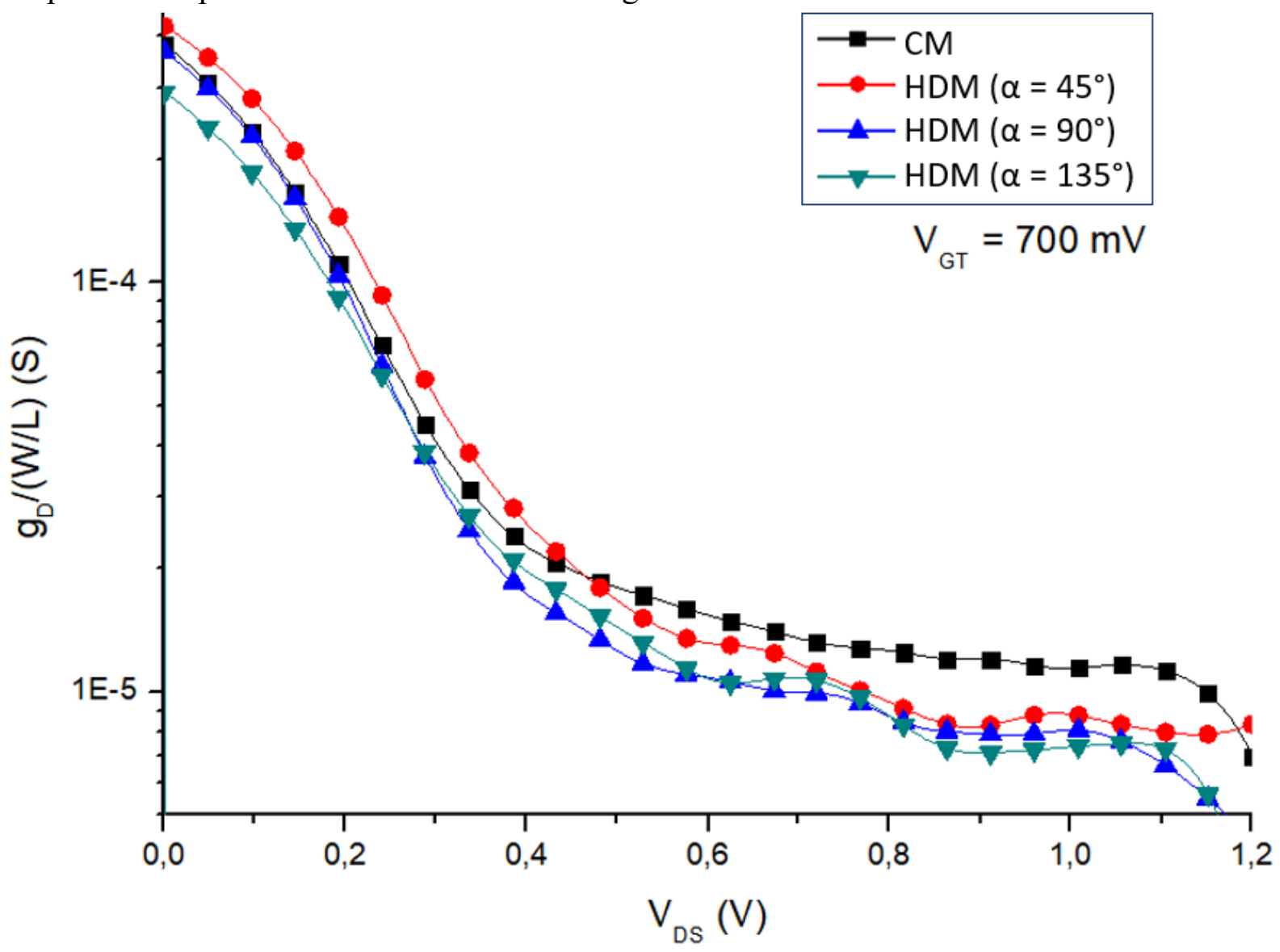

Fonte: Autor

Analisando-se as curvas da Figura 36, para uma $\mathrm{V}_{\mathrm{DS}}$ igual a $700 \mathrm{mV}$ e VGT igual a 700 $\mathrm{mV}$ verifica-se que a $\mathrm{g}_{\mathrm{D}} /(\mathrm{W} / \mathrm{L})$ do $\mathrm{CM}$ é igual a $13,48 \mu \mathrm{S}$, para o $\mathrm{HDM}\left(\alpha=45^{\circ}\right)$ o $\mathrm{gD}_{\mathrm{D}} /(\mathrm{W} / \mathrm{L})$ é igual a $11,68 \mu \mathrm{S}$, para a $\mathrm{g}_{\mathrm{D}} /(\mathrm{W} / \mathrm{L})$ do $\operatorname{HDM}\left(\alpha=90^{\circ}\right)$ é igual a $9,94 \mu \mathrm{S}$ e o $\mathrm{HDM}\left(\alpha=135^{\circ}\right)$ apresentou uma $\mathrm{gD}_{\mathrm{D}} /(\mathrm{W} / \mathrm{L})$ igual a $10,8 \mu \mathrm{S}$. Esses valores são justificados porque os HDMs apresentaram maiores valores de comprimento efetivo de canal em relação ao do $\mathrm{CM}$ equivalente, reduzindo desta forma o efeito de modulação de canal [1].

\subsection{VALORES DE VEA E Av DOS MOSFETS COM LEIAUTES DE PORTA DOS TIPOS DIAMANTE HÍBRIDO E DO TIPO RETANGULAR}

Uma vez obtidas as curvas de $\mathrm{g}_{\mathrm{m}} /(\mathrm{W} / \mathrm{L})$ e $\mathrm{g}_{\mathrm{D}} /(\mathrm{W} / \mathrm{L})$ para diferentes $\mathrm{V}_{\mathrm{GTS}}$ e considerandose um VDS igual a $700 \mathrm{mV}$, é possível obter o Av, por meio da Equação (5).

$\mathrm{Na}$ Tabela 5 são apresentados os valores dos Avs dos MOSFETs dos tipos Diamante Híbrido e retangular para diferentes valores de $\mathrm{V}_{\mathrm{GT}}$. 
Tabela 5 - Valores dos ganhos de tensão em função de $V_{\text {GT }}$ dos HDMs $\left(\alpha=45^{\circ} ; 90^{\circ} ; 135^{\circ}\right)$ e o CM equivalente, considerando-se uma $\mathrm{VDS}$ igual a $700 \mathrm{mV}$.

\begin{tabular}{c|c|c|c|c}
\hline $\begin{array}{c}\mathbf{V}_{\mathbf{G T}} \\
{[\mathrm{V}]}\end{array}$ & $\mathbf{A v} \mathbf{C M}[\mathbf{d B}]$ & $\mathbf{A v} \mathbf{H D M}\left(\boldsymbol{\alpha}=\mathbf{4 5}^{\circ}\right)[\mathbf{d B}]$ & Av HDM $\left(\boldsymbol{\alpha}=\mathbf{9 0}^{\circ}\right)[\mathbf{d B}]$ & Av HDM $(\boldsymbol{\alpha}=\mathbf{1 3 5})[\mathbf{d B}]$ \\
\hline 0,1 & 27,06 & 33,98 & 32,66 & 29,77 \\
\hline 0,2 & 26,11 & 31,47 & 29,54 & 27,97 \\
\hline 0,3 & 25,18 & 29,78 & 28,82 & 27,43 \\
\hline 0,4 & 23,02 & 28,07 & 26,82 & 25,69 \\
\hline 0,5 & 21,03 & 26,27 & 25,19 & 23,94 \\
\hline 0,6 & 19,55 & 24,85 & 23,59 & 22,56 \\
\hline 0,7 & 18,66 & 23,57 & 22,44 & 21,74 \\
\hline
\end{tabular}

Fonte: Autor

Analisando a Tabela 5, observa-se que para toda a extensão da $\mathrm{V}_{\text {GT }}$ estudada $(0,1 \mathrm{~V} \leq$ $\mathrm{V}_{\mathrm{GT}} \leq 0,7 \mathrm{~V}$ ) os HDMs apresentaram maiores valores de Av em relação ao CM. Os HDMs apresentaram valores máximos de Av para a $\mathrm{V}_{\mathrm{GT}}$ igual a $0,1 \mathrm{~V}$. O Av de todos os dispositivos, à medida que aumentava a $V_{G T}$, teve seu valor reduzido. Isso pode ser justificado por meio da Equação (5) e das curvas ilustradas na Figura 34, porque, à medida que aumenta o valor de $\mathrm{V}_{\mathrm{GT}}$, o valor de $g_{m} / I_{D s}$ e os dispositivos caminham para a região de inversão forte que é caracterizado por apresentarem um baixo Av e uma ótima resposta em frequência. Os HDMs apresentaram maiores valores de Av pois apresentaram maiores valores de transcondutâncias ou menores valores de $g_{D}$ em função da presença do LCE e do PAMDLE quando comparados aos do CM equivalente.

Logo, podemos concluir que os HDMs podem ser usados no lugar de CMs equivalentes para aumentar ainda mais os Avs de CIs CMOS analógicos (amplificadores operacionais de transcondutância, OTA, etc.), em especial o $\operatorname{HDM}\left(\alpha=45^{\circ}\right)$ por apresentar maior Av entre os dispositivos pesquisados.

Na Figura 37 são apresentadas as curvas do ganho de tensão dos MOSFETs dos tipos Diamante Híbrido e retangular em função da sobretensão de porta [Figura 37.(a)] e em função da IDS/(W/L) [Figura 37.(b)] para uma $\mathrm{V}_{\mathrm{DS}}$ igual a $700 \mathrm{mV}$. 
Figura 37 - Curvas de Av em função da $V_{G T}$ (a) e IDS/(W/L) (b) dos MOSFETs com leiautes de porta dos tipos Diamante Híbrido e retangular, para um $\mathrm{V}_{\mathrm{DS}}=700 \mathrm{mV}$.
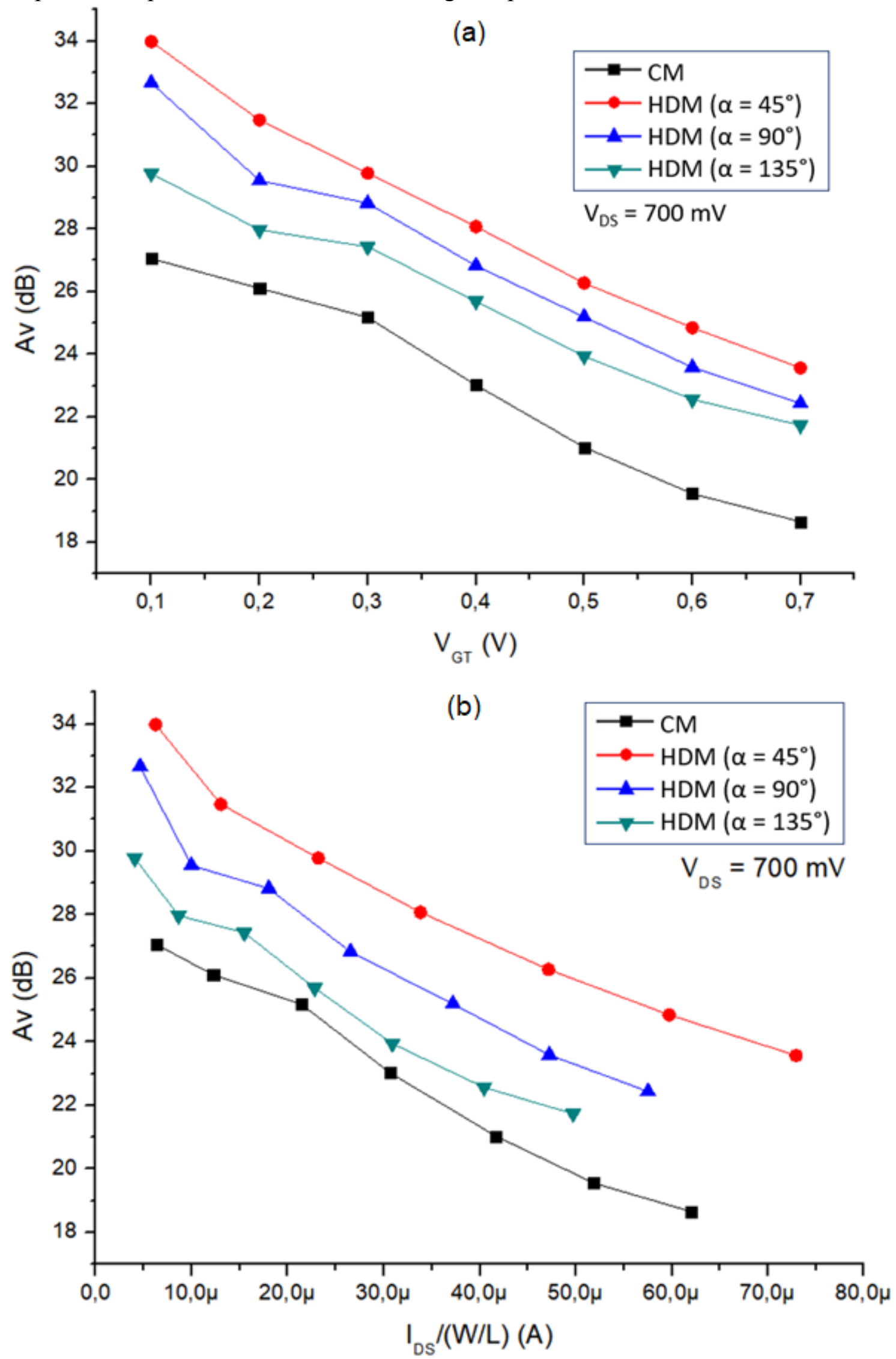

Fonte: Autor 
Para obter os valores da VEA dos MOSFETs foi utilizada a Equação (37) [2].

$$
V_{E A}=\frac{I_{D S} /(W / L)}{g_{D} /(W / L)}[V]
$$

Assim, a partir dos valores da IDs/(W/L) e da $\mathrm{g}_{\mathrm{D}} /(\mathrm{W} / \mathrm{L})$ e utilizando-se a Equação (37), pode-se obter os valores de $\mathrm{V}_{\mathrm{EA}}$ dos diferentes MOSFETs estudados, levando-se em conta diferentes valores de $\mathrm{V}_{\mathrm{GT}}$ e considerando-se uma $\mathrm{V}_{\mathrm{DS}}$ de $700 \mathrm{mV}$. Esses valores de $\mathrm{V}_{\mathrm{EA}}$ estão indicados na Tabela 6.

Tabela 6 - Valores das tensões de Early em função da sobretensão de porta dos HDMs $(\alpha=$ $45^{\circ} ; 90^{\circ} ; 135^{\circ}$ ) e o $\mathrm{CM}$ equivalente, considerando-se uma $\mathrm{V}_{\mathrm{DS}}$ igual a $700 \mathrm{mV}$.

\begin{tabular}{c|c|c|c|c}
\hline $\begin{array}{c}\mathbf{V}_{\text {GT }} \\
{[\mathrm{V}]}\end{array}$ & VEA CM [V] & VEA HDM $\left(\boldsymbol{\alpha}=\mathbf{4 5}^{\circ}\right)$ [V] & VEA HDM $\left(\boldsymbol{\alpha}=\mathbf{9 0}^{\circ}\right)$ [V] & VEA HDM $\left(\boldsymbol{\alpha}=\mathbf{1 3 5}^{\circ}\right)[\mathbf{V}]$ \\
\hline 0,1 & 2,44 & 5,29 & 4,41 & 3,49 \\
\hline 0,2 & 3,33 & 6,09 & 4,73 & 4,00 \\
\hline 0,3 & 4,23 & 6,82 & 6,00 & 5,11 \\
\hline 0,4 & 4,33 & 7,26 & 6,24 & 5,46 \\
\hline 0,5 & 4,49 & 7,63 & 6,58 & 5,74 \\
\hline 0,6 & 4,62 & 7,82 & 6,68 & 5,95 \\
\hline 0,7 & 4,98 & 7,98 & 7,22 & 6,55 \\
\hline
\end{tabular}

Fonte: Autor

Analisando a Tabela 6, observa-se que para toda a extensão de $\mathrm{V}_{\text {GT }}$ estudada, os HDMs apresentaram maiores valores de $\mathrm{V}_{\mathrm{EA}}$ em relação ao CM. Isso é justificado pois os HDMs com diferentes $\alpha$ nas faixas de $\mathrm{V}_{\mathrm{GT}}$ consideradas, ou apresentaram maiores valores de $\mathrm{IDS}_{\mathrm{DS}}(\mathrm{W} / \mathrm{L})$, como no caso do $\operatorname{HDM}\left(\alpha=45^{\circ}\right)$, ou menores valores de $g_{D}$ em função da presença dos efeitos do LCE e do PAMDLE quando comparados aos do CM equivalente.

Logo podemos concluir que os HDMs, podem ser usados no lugar de CMs equivalentes para aumentar a $\mathrm{V}_{\mathrm{EA}}$ de CIs CMOS analógicos, em especial o $\mathrm{HDM}\left(\alpha=45^{\circ}\right)$, por apresentar maior valor de $\mathrm{V}_{\mathrm{EA}}$ entre os dispositivos pesquisados.

A Figura 38 apresenta as curvas das tensões Early dos MOSFETs dos tipos Diamante Híbrido e retangular em função da sobretensão de porta, para VDs igual a $700 \mathrm{mV}$ e diferentes valores de $\mathrm{V}_{\mathrm{GT}}$. 
Figura 38 - Tensão Early em função da sobretensão de porta experimental dos MOSFETs com leiautes de porta dos tipos Diamante Híbrido e o retangular, para uma VDs igual a $700 \mathrm{mV}$.

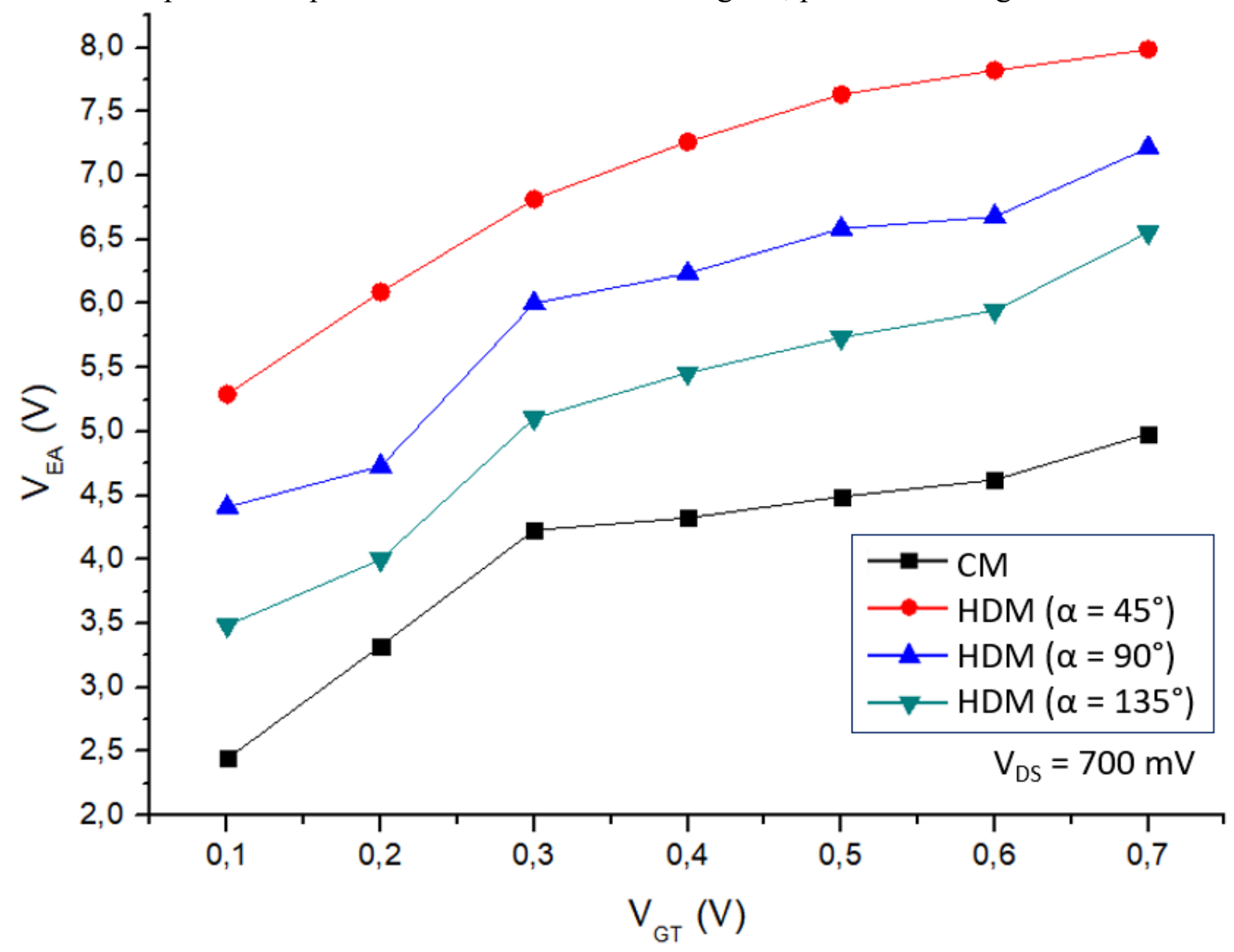

Fonte: Autor

\subsection{DISTORÇÕES HARMÔNICAS PARA OS MOSFETS OPERANDO NA REGIÃO DE SATURAÇÃO}

A distorção harmônica total, distorção da segunda harmônica e distorção da terceira harmônica dos MOSFETs dos tipos Diamante Híbridos e do retangular são analisados através do IFM, utilizando um grau do polinômio de aproximação igual a 5, suportado pela ferramenta matemática Mathcad 14.0. Para a análise da distorção harmônica, os MOSFETs foram considerados como amplificadores de tensão baseados em um único transistor, como foi ilustrado na Figura 11.

\subsubsection{Distorção Harmônica Total (THD)}

Em relação à THD dos dispositivos, a Figura 39 mostra as curvas dos MOSFETs estudados nesse projeto de pesquisa, em função da $V_{G T}$ [Figura 39.(a)] e em função de $g_{m} / I_{D S}$ 
[Figura 39.(b)], considerando uma tensão do sinal de entrada igual a $50 \mathrm{mV}, \mathrm{V}_{\mathrm{DS}}$ de $700 \mathrm{mV}$ e com variação do seu nível DC de $\mathrm{V}_{\mathrm{TH}}$ para $\mathrm{V}_{\mathrm{TH}}+0,7 \mathrm{~V}\left(0 \mathrm{~V} \leq \mathrm{V}_{\mathrm{GT}} \leq 0,7 \mathrm{~V}\right)$.

Figura 39 - Curvas da THD em função de $V_{G T}$ (a) e em função $g_{m} / I_{D S}$ (b) dos MOSFETs, considerando uma $\mathrm{V}_{\mathrm{DS}}$ igual a $700 \mathrm{mV}$, Va igual a $50 \mathrm{mV}$ e com $0 \mathrm{~V} \leq \mathrm{V}_{\mathrm{GT}} \leq 0,7 \mathrm{~V}$.
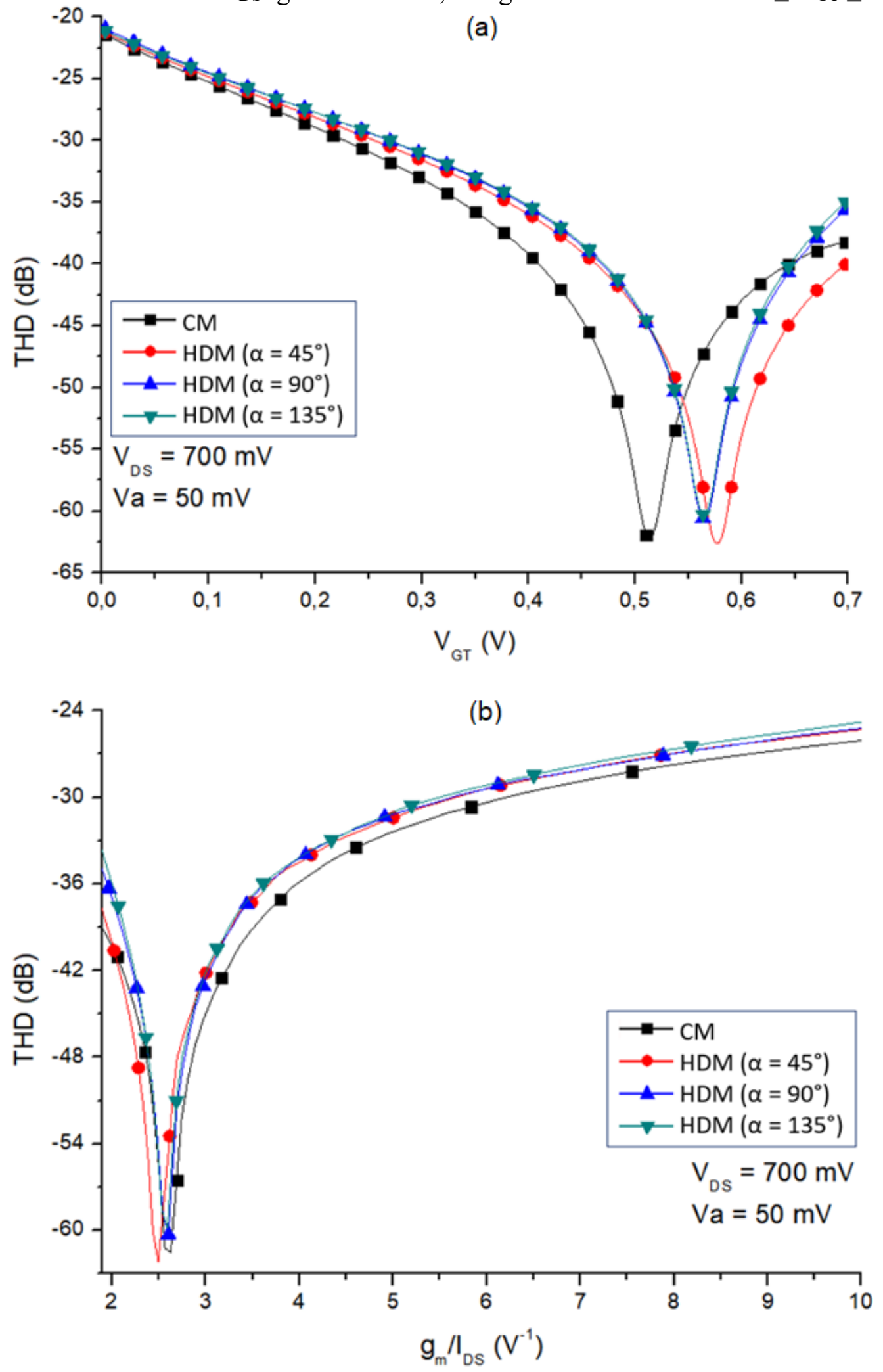

Fonte: Autor 
Analisando as curvas presentes na Figura 39.(a) notamos que, a medida que o valor da VGT aumenta, os MOSFETs passam a operar na região de triodo e no regime de inversão de canal forte, de maneira inversa, quanto menor a $V_{\text {GT }}$ os dispositivos passam a operar na região de saturação e no regime de inversão fraca do canal.

Observa-se que, para valores de $\mathrm{V}_{\mathrm{GT}}$ maiores ou iguais a $0,55 \mathrm{~V}$, o dispositivo que apresenta maior linearidade, isto é, menor distorção harmônica, é o HDM com ângulo $\alpha$ igual a $45^{\circ}$, com aproximadamente uma THD $11,15 \mathrm{~dB}$ menor que à do $\mathrm{CM}$, para uma $\mathrm{V}_{\text {GT }}$ igual a $0,6 \mathrm{~V}$.

Já para valores de $\mathrm{V}_{\mathrm{GT}}$ menores que 0,55 V, o MOSFET que apresentou maior linearidade foi o CM equivalente, com aproximadamente uma distorção harmônica total 17,57 $\mathrm{dB}$ menor do que o medido no $\mathrm{HDM}$ com ângulo $\alpha$ igual a $45^{\circ}$, para uma $\mathrm{V}_{\mathrm{GT}}$ igual a $0,51 \mathrm{~V}$.

$\mathrm{Na}$ Figura 39.(a) os picos presentes nas curvas da THD dos HDMs e do CM correspondem à transcondutância máxima em cada um dos transistores, ou seja, ao ponto de máxima mobilidade dos portadores [36].

A Figura 39.(b) ilustra as curvas da THD em função de $\mathrm{g}_{\mathrm{m}} / \mathrm{I}_{\mathrm{DS}}$ dos MOSFETs. Essa figura de mérito é obtida para poder ter uma melhor visualização da região de interesse da curva.

A redução na distorção harmônica pode ser modelada por meio da análise da derivada de primeira ordem de $g_{m}$ em função de $V_{G T}$ [4][36]. Essa figura de mérito pode ser determinada analiticamente por meio da Equação (38) [4].

$$
T H D \cong H D 2=\frac{1}{2} V a \frac{\frac{\partial g_{m}}{\partial V_{G T}}}{2 g_{m}}
$$

Esta comparação é válida porque, ao considerar o MOSFET polarizado como um amplificador para baixas amplitudes de sinal senoidal aplicado à sua entrada, em saturação o transistor é dominado pela HD2 [4], como observado na seguinte equação (38). Ao se efetuar o cálculo de HD2, percebe-se que a variação da $g_{m}$ (Figura 40) dos MOSFETs é a principal responsável pela melhora na linearidade, na qual uma maior variação de $g_{m}$ resulta numa menor THD, o que explica a melhora da THD do CM em relação aos HDMs durante o intervalo de $\mathrm{V}_{\mathrm{GT}}$ entre $0,0 \mathrm{~V}$ a $0,55 \mathrm{~V}$. A melhora observada na THD dos HDMs com o aumento da $\mathrm{V}_{\mathrm{GT}}$ $\left(\mathrm{V}_{\mathrm{GT}}>0,55 \mathrm{~V}\right)$ é devido à maior resistência série do $\operatorname{HDM}\left(\alpha=90^{\circ}\right)$ e do $\operatorname{HDM}\left(\alpha=135^{\circ}\right)$ e a maior $\mathrm{g}_{\mathrm{m}}$ do $\operatorname{HDM}\left(\alpha=45^{\circ}\right)$, em relação ao $\mathrm{CM}$, já que a partir desse ponto de $\mathrm{V}_{\mathrm{GT}}$ a $\mathrm{dg}_{\mathrm{m}} /(\mathrm{W} / \mathrm{L}) / \mathrm{dV}_{\mathrm{GT}}$ do CM torna-se praticamente igual aos demais HDMs. 
Figura 40 - Curvas da dgm/(W/L)/dV GT em função de $V_{\text {GT }}$ dos HDMs e do CM, para uma VDS igual a $700 \mathrm{mV}$.

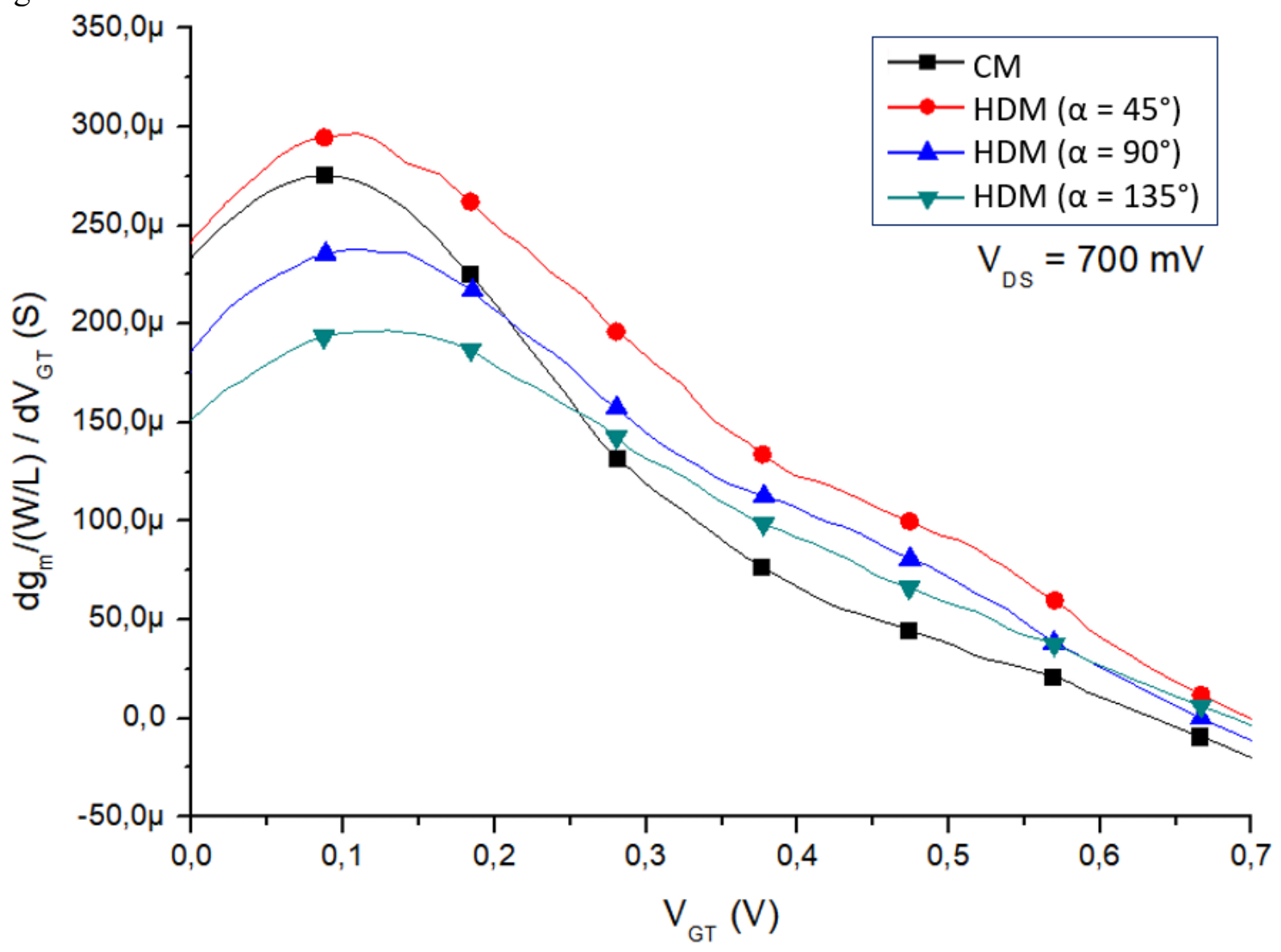

Fonte: Autor

\subsubsection{Distorção Harmônica Total normalizada pelo ganho de tensão (THD/Av)}

Dada a relação do ganho de tensão com a não-linearidade [4][35], para a correta obtenção da distorção harmônica de um dispositivo, é necessária a normalização de THD por Av [4], que é expressa por meio da Equação (39).

$$
\frac{T H D}{A v}=\frac{1}{2} V a \frac{\frac{\partial g_{m}}{\partial V_{G T}}}{2 g_{m}^{2}} g_{D}
$$

Em relação à THD/Av dos dispositivos, a Figura 41 mostra as curvas dos MOSFETs estudados nesse projeto de pesquisa em função da $V_{G T}$ [Figura 41.(a)] e em função da $g_{m} / I_{D S}$ [Figura 41.(b)], considerando uma Va de $50 \mathrm{mV}$, $\mathrm{V}_{\mathrm{DS}}$ de $700 \mathrm{mV}$ e com variação do seu nível $\mathrm{DC}$ de $\mathrm{V}_{\mathrm{TH}}+0,1 \mathrm{~V}$ para $\mathrm{V}_{\mathrm{TH}}+0,7 \mathrm{~V}\left(0,1 \mathrm{~V} \leq \mathrm{V}_{\mathrm{GT}} \leq 0,7 \mathrm{~V}\right)$. 
Figura 41 - THD/Av em função de $V_{\text {GT }}$ e em função de $g_{m} / I_{D S}$ dos MOSFETs dos tipos Diamante Híbrido com ângulos iguais a $45^{\circ}, 90^{\circ}$ e $135^{\circ}$ e do retangular para um Va igual a 50 $\mathrm{mV}$ (a) e uma $\mathrm{V}_{\mathrm{DS}}$ igual a $700 \mathrm{mV}$ (b).
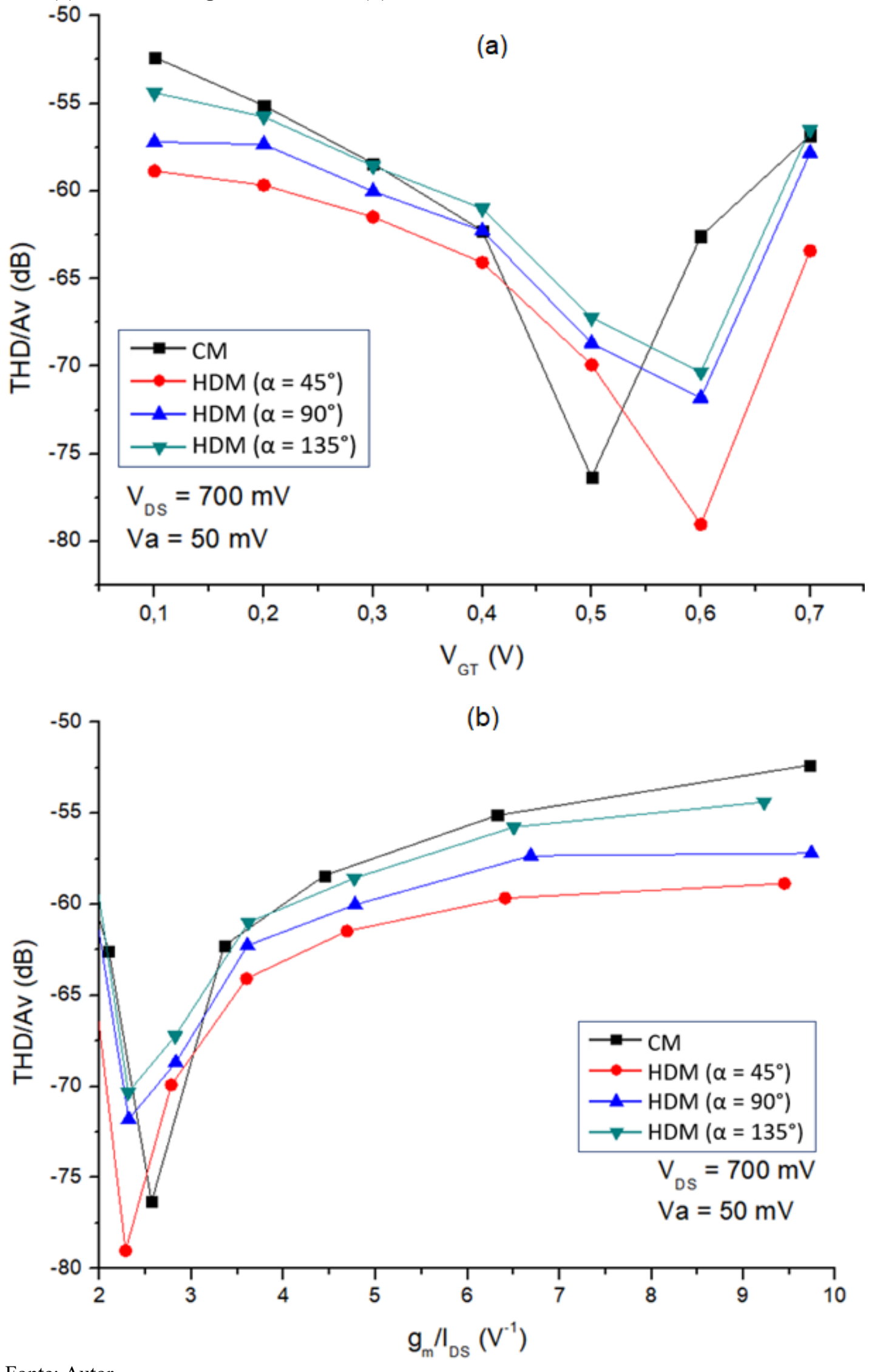

Fonte: Autor 
Examinando a Figura 41.(a), pode-se notar que a THD/Av dos HDMs é menor do que a do CM equivalente durante os intervalos de $\mathrm{V}_{\mathrm{GT}}$ entre $0,1 \mathrm{~V}$ a $0,3 \mathrm{~V}$ e $0,6 \mathrm{~V}$ a $0,7 \mathrm{~V}$, em que o $\operatorname{HDM}\left(\alpha=45^{\circ}\right)$ apresentou uma THD/Av $16,45 \mathrm{~dB}$ menor que à do CM, para uma $\mathrm{V}_{\mathrm{GT}}$ igual a 0,6 V. O CM apresentou menor THD/Av durante o intervalo de $\mathrm{V}_{\mathrm{GT}}$ de $0,4 \mathrm{~V}$ a $0,5 \mathrm{~V}$, isso é justificado, pois nesse intervalo de $\mathrm{V}_{\mathrm{GT}}$ o $\mathrm{CM}$ apresentou valores de THD e Av capazes de promoverem uma THD/Av menor que os demais dispositivos.

Quando analisada a Figura 41.(b) nota-se que para valores de $g_{m} / I_{D s}$ acima de $3 \mathrm{~V}^{-1}$ os HDMs apresentaram menores valores de THD/Av em relação ao CM, em especial o HDM ( $\alpha$ $=45^{\circ}$ ) que apresentou um valor de $\mathrm{THD} / \mathrm{Av} 6,49 \mathrm{~dB}$ menor que a do CM equivalente, para uma $\mathrm{gm} / \mathrm{IDs}$ igual a $9,4 \mathrm{~V}^{-1}$.

Isso pode ser justificado pois na inversão de canal forte $\left(\mathrm{g}_{\mathrm{m}} / \mathrm{I}_{\mathrm{DS}}\right.$ menor ou igual a $10 \mathrm{~V}^{-}$

$\left.{ }^{1}\right)$ os HDMs apresentaram maiores valores de Av em relação ao CM, melhorando, dessa maneira, a figura de mérito de THD/Av. Essa melhora de Av é resultado do LCE e o PAMDLE presentes na estrutura do $\operatorname{HDM}\left(\alpha=45^{\circ}\right)$, que são responsáveis por aumentar a $\mathrm{g}_{\mathrm{m}} /(\mathrm{W} / \mathrm{L})$ e a melhora do $\operatorname{HDM}\left(\alpha=90^{\circ}\right)$ e do $\operatorname{HDM}\left(\alpha=135^{\circ}\right)$ é devida à $\mathrm{g}_{\mathrm{D}} /(\mathrm{W} / \mathrm{L})$ desses dispositivos que apresentaram menores valores quando comparados ao $\mathrm{CM}$ e ao $\operatorname{HDM}\left(\alpha=45^{\circ}\right)$ [4][13].

Logo pode-se concluir que os HDMs são opções alternativas para a substituição de CMs equivalentes a fim de aumentar a linearidade de CIs CMOS analógicos em aplicações em alta frequência, em especial o $\operatorname{HDM}\left(\alpha=45^{\circ}\right)$ por apresentar a menor THD/Av entre os HDMs.

\subsubsection{Distorção Harmônica Total normalizada pelo ganho de tensão em função da amplitude do sinal de entrada senoidal (Va)}

Com o objetivo de verificar o comportamento da linearidade em função da amplitude de um sinal senoidal de entrada variando entre $0 \mathrm{~V}$ a $100 \mathrm{mV}$, considerando uma VDs igual a $700 \mathrm{mV}$ e o valor de $\mathrm{V}_{\mathrm{GT}}$ fixado em $600 \mathrm{mV}$, pois nesse ponto os HDMs apresentaram uma menor THD/Av, conforme verificado na Figura 40.(a).

A Figura 42 mostra a THD/Av em função de Va para os MOSFETs do Diamante Híbrido com diferentes ângulos e o retangular. 
Figura 42 - Curva experimental de THD/Av em função de Va dos MOSFETs dos tipos Diamante Híbrido com ângulos iguais a $45^{\circ}, 90^{\circ}$ e $135^{\circ}$ e do retangular operando com um $\mathrm{V}_{\mathrm{GT}}$ igual a $600 \mathrm{mV}$ e um $\mathrm{V}_{\mathrm{DS}}$ igual a $700 \mathrm{mV}$.

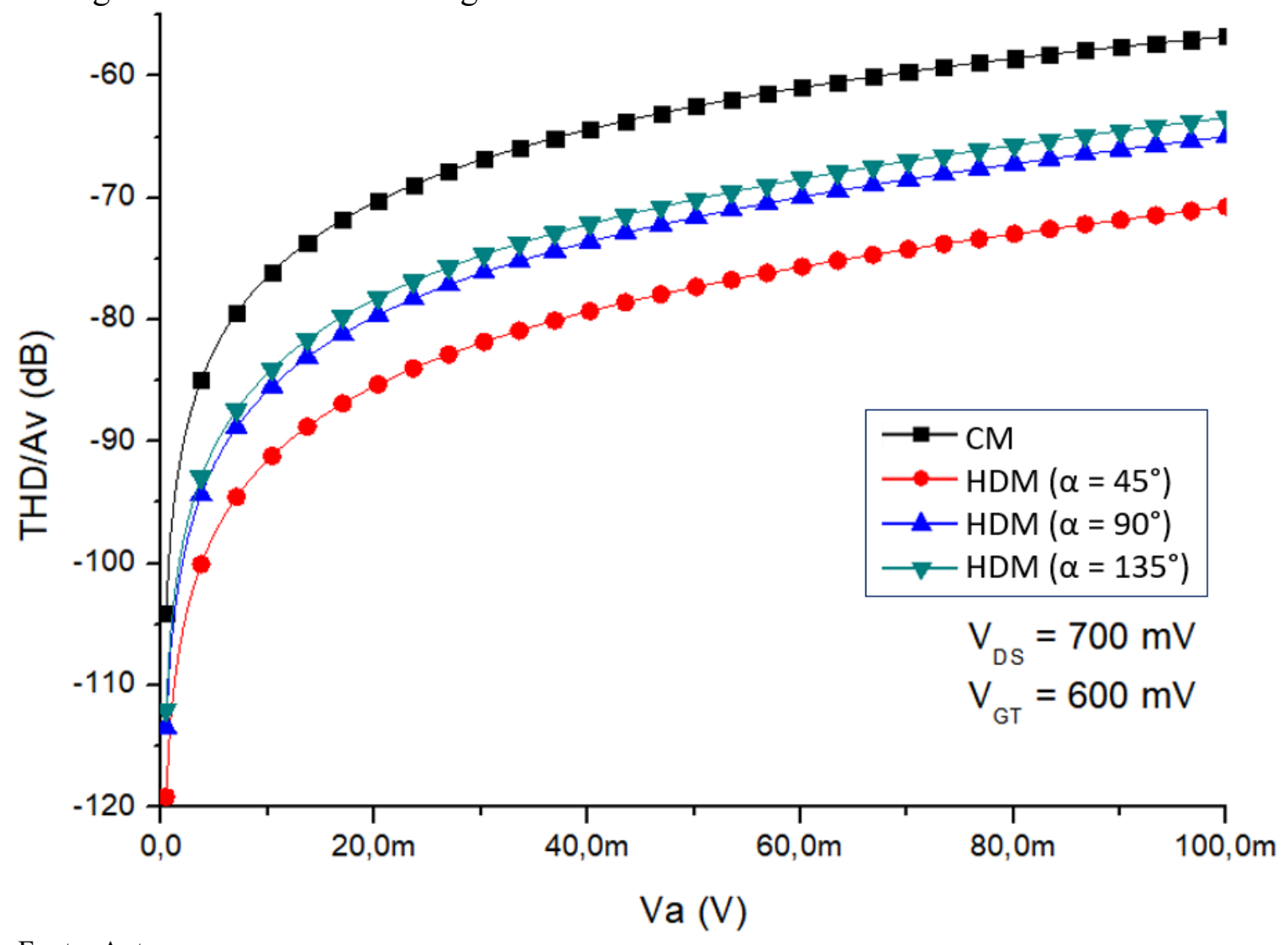

Fonte: Autor

Observa-se na Figura 42 que para Va igual a $50 \mathrm{mV}$ a THD/Av do HDM $\left(\alpha=45^{\circ}\right)$ apresentou uma THD/Av cerca de 14,84 dB menor em relação ao valor do CM equivalente. $\mathrm{O}$ mesmo comportamento é verificado para a THD/Av do HDM $\left(\alpha=90^{\circ}\right)$ que exibiu uma THD/Av em torno de 9,12 dB menor em comparação à do CM equivalente e a THD/Av do $\operatorname{HDM}\left(\alpha=135^{\circ}\right)$ apresentou um valor cerca de $7,65 \mathrm{~dB}$ menor em relação ao valor do $\mathrm{CM}$ equivalente.

Isso é justificado pelo fato de que os HDMs, durante o intervalo de $\mathrm{V}_{\mathrm{GT}}$ entre $0,6 \mathrm{~V}$ a $0,7 \mathrm{~V}$, apresentaram uma menor THD/Av em relação ao CM.

Essa figura de mérito é interessante de se obter, visto que, através dela pode-se determinar a amplitude de um sinal senoidal de entrada para uma determinada THD/Av [4]. Encontramos estes valores presentes na Tabela 7. 
Tabela 7 - THD/Av em função da Va dos MOSFETs dos tipos Diamantes Híbridos e retangular para um valor de $\mathrm{V}_{\mathrm{DS}}$ igual a $700 \mathrm{mV}$ e operando com uma $\mathrm{V}_{\mathrm{GT}}$ igual a $600 \mathrm{mV}$.

\begin{tabular}{c|cccc}
\hline THD/Av [dB] & Va CM [mV] & $\begin{array}{c}\text { Va HDM }\left(\boldsymbol{\alpha}=\mathbf{4 5}^{\circ}\right) \\
{[\mathbf{m V}]}\end{array}$ & $\begin{array}{c}\text { Va HDM }\left(\boldsymbol{\alpha}=\mathbf{9 0}^{\circ}\right) \\
{[\mathbf{m V}]}\end{array}$ & $\begin{array}{c}\mathbf{V a ~ H D M ~}\left(\boldsymbol{\alpha}=\mathbf{1 3 5}^{\circ}\right) \\
{[\mathbf{m V}]}\end{array}$ \\
\hline-105 & 0,41 & 2,07 & 1,24 & 0,83 \\
\hline-100 & 0,83 & 3,37 & 2,07 & 1,66 \\
\hline-95 & 1,24 & 6,64 & 3,31 & 2,90 \\
\hline-90 & 2,07 & 12,03 & 6,22 & 5,39 \\
\hline
\end{tabular}

Fonte: Autor

Verificando a Tabela 7, nota-se que, para uma mesma THD/Av (THD/Av $=-90 \mathrm{~dB}), \mathrm{o}$ $\operatorname{HDM}\left(\alpha=45^{\circ}\right)$ pode amplificar sinais senoidais com amplitudes de até 5,81 vezes maiores que as suportadas pelo o $\mathrm{CM}$ equivalente, o $\operatorname{HDM}\left(\alpha=90^{\circ}\right)$ foi capaz de amplificar sinais até 3 vezes maiores que as suportadas pelo o $\mathrm{CM}$ e o $\operatorname{HDM}\left(\alpha=135^{\circ}\right)$ pode amplificar sinais senoidais de amplitudes 2,6 vezes maiores que as suportadas pelo o CM equivalente, quando esses se encontram na região de saturação. 


\section{CONCLUSÃO}

Este trabalho teve por objetivo estudar a distorção harmônica (linearidade) por meio de resultados experimentais do MOSFET com tecnologia Bulk e leiaute de porta do tipo Diamante Híbrido com ângulos $\alpha$ de $45^{\circ}\left[\operatorname{HDM}\left(\alpha=45^{\circ}\right)\right], 90^{\circ}\left[\operatorname{HDM}\left(\alpha=90^{\circ}\right)\right]$ e $135^{\circ}[\operatorname{HDM}(\alpha=$ $\left.135^{\circ}\right)$ ] e com leiaute de porta retangular $(\mathrm{CM})$.

Quando analisamos a curva característica IDS/(W/L) em função da VGT, obtida experimentalmente para os MOSFETs estudados por essa dissertação de mestrado, percebe-se que o $\operatorname{HDM}\left(\alpha=45^{\circ}\right)$ possui uma IDS/(W/L) maior que o do CM, em que, para o valor de $V_{G T}$ igual a $0,7 \mathrm{~V}$, a IDs/(W/L) foi $17,65 \%$ maior que à do $\mathrm{CM}$, porém para os HDMs com ângulos iguais a $90^{\circ}$ e $135^{\circ}$, o valor da IDs/(W/L) foi menor que o valor da IDS/(W/L) do CM. Isso se deve aos LCE e PAMDLE que no $\operatorname{HDM}\left(\alpha=45^{\circ}\right)$ foram capazes de compensar a degradação da corrente entre o dreno e a fonte, mesmo tendo uma W/L maior $(3,94)$ que a do $\mathrm{CM}$ equivalente $(2,33)$ e os HDMs com $\alpha$ iguais a $90^{\circ}$ e $135^{\circ}$, apesar de possuírem em suas estruturas o LCE e o PAMDLE, não foram capazes de compensar suas maiores W/L em relação à do $\mathrm{CM}$ equivalente.

A partir da curva de IDS/(W/L), em função de $\mathrm{V}_{\text {GT }}$ com uma $\mathrm{V}_{\mathrm{DS}}$ igual a $50 \mathrm{mV}$, foi determinado a $\mathrm{R}_{S} *(\mathrm{~W} / \mathrm{L})$ dos MOSFETs. É necessário que seja determinada a $\mathrm{R}_{\mathrm{S}} *(\mathrm{~W} / \mathrm{L})$ nessas condições para que a $\mathrm{RSD}_{\mathrm{SD}}(\mathrm{W} / \mathrm{L})$ seja praticamente igual à $\mathrm{R}_{\mathrm{S}} *(\mathrm{~W} / \mathrm{L})$. Para uma $\mathrm{V}_{\mathrm{GT}}$ igual a 0,7 $\mathrm{V}$ o $\operatorname{HDM}\left(\alpha=45^{\circ}\right)$ possui uma $\left.\mathrm{Rs}_{\mathrm{s}} * \mathrm{~W} / \mathrm{L}\right)$ igual a $3,98 \mathrm{k} \Omega$, o $\mathrm{CM}$ possui uma $\mathrm{Rs}^{*}(\mathrm{~W} / \mathrm{L})$ igual a 4,07 k $\Omega$, o $\operatorname{HDM}\left(\alpha=90^{\circ}\right)$ possui uma $\mathrm{Rs}_{\mathrm{S}}(\mathrm{W} / \mathrm{L})$ igual a $4,65 \mathrm{k} \Omega$ e o $\operatorname{HDM}\left(\alpha=135^{\circ}\right)$ possui uma $\mathrm{Rs}^{*}(\mathrm{~W} / \mathrm{L})$ igual a $5,60 \mathrm{k} \Omega$. Isso é justificado a pelo LCE e o PAMDLE presentes na estrutura do $\operatorname{HDM}\left(\alpha=45^{\circ}\right)$ que é responsável por aumentar a IDS/(W/L) e dessa forma capaz de reduzir a $\mathrm{Rs}^{*}(\mathrm{~W} / \mathrm{L})$, enquanto no $\operatorname{HDM}\left(\alpha=90^{\circ}\right)$ e no $\operatorname{HDM}\left(\alpha=135^{\circ}\right)$ isso não ocorre, fazendo com que as suas IDS/(W/L) sejam menores do que a do CM e, dessa forma, aumentando a $\mathrm{RS}_{\mathrm{S}}(\mathrm{W} / \mathrm{L})$.

Realizando a derivada das curvas $\mathrm{IDS}_{\mathrm{DS}}(\mathrm{W} / \mathrm{L})$ em função de $\mathrm{V}_{\mathrm{GT}}$ extrai-se a transcondutância dos dispositivos. Na análise da $\mathrm{g}_{\mathrm{m}} /(\mathrm{W} / \mathrm{L})$ o $\mathrm{HDM}\left(\alpha=45^{\circ}\right)$ apresentou um valor cerca de $27,98 \%$ maior que à do $\mathrm{CM}$ equivalente, o $\mathrm{HDM}\left(\alpha=90^{\circ}\right)$ possui uma $\mathrm{g}_{\mathrm{m}} /(\mathrm{W} / \mathrm{L})$ praticamente igual à do $\mathrm{CM}$ e já para a $\mathrm{g}_{\mathrm{m}} /(\mathrm{W} / \mathrm{L})$ do $\operatorname{HDM}\left(\alpha=135^{\circ}\right)$ teve um valor cerca de $13,11 \%$ menor que a $\mathrm{gm}_{\mathrm{m}} /(\mathrm{W} / \mathrm{L})$ do CM. Justificado pelos valores de IDS/(W/L), uma vez que a $\mathrm{g}_{\mathrm{m}} /(\mathrm{W} / \mathrm{L})$ é obtido por meio da primeira derivada da curva de IDS/(W/L) em função de VGT. 
Ao fazer a curva IDS/(W/L) em função de $\mathrm{V}_{\mathrm{GT}}$ em escala logarítmica, para um VDS igual a $700 \mathrm{mV}$, foi possível obter a inclinação de sublimiar dos MOSFETs, em que as SS do CM e do $\operatorname{HDM}\left(\alpha=90^{\circ}\right)$ foram praticamente idênticas, com apenas uma variação entre si de $4 \%$, dado que, foram manufaturados com a mesma tecnologia de CI CMOS. O HDM $\left(\alpha=45^{\circ}\right)$ e o $\operatorname{HDM}\left(\alpha=135^{\circ}\right)$ possuíram valores de SS cerca de $11,09 \%$ e 57,03\%, respectivamente. Explicase essa característica devido à degradação da corrente de estado desligado que foi muito elevada em ambos os MOSFETs.

Considerando uma VGt igual a $-0,6 \mathrm{~V}$ a Ioff/(W/L) do $\operatorname{HDM}\left(\alpha=45^{\circ}\right)$ e do HDM $(\alpha=$ $135^{\circ}$ ) foram 14,35 e 10,18 vezes menor, respectivamente, em relação à do CM. Isso ocorre porque os HDMs $\left(\alpha=45^{\circ} ; 135^{\circ}\right)$ apesar de possuírem maiores Leffs em comparação ao CM equivalente apresentaram um perímetro da junção p-n maior que o do CM equivalente, e o seus LEFs não foram capazes se sobrepor a esse efeito de perímetro, dessa forma, as suas correntes de fuga foram maiores que a do $\mathrm{CM}$ equivalente, já o $\operatorname{HDM}\left(\alpha=90^{\circ}\right)$ apresentou uma Ioff/(W/L) menor, cerca de 68,7\%, que a do CM equivalente, porque, apesar de apresentar um perímetro da junção p-n maior que a do CM equivalente, não sofreu degradação do seu campo elétrico longitudinal, que é o responsável por aumentar o comprimento da região de depleção do dispositivo.

Analisando as curvas de $\mathrm{g}_{\mathrm{m}} / \mathrm{I}_{\mathrm{DS}}$ em função de $\mathrm{IDS}_{\mathrm{DS}}(\mathrm{W} / \mathrm{L})$ dos MOSFETs para $\mathrm{V}_{\mathrm{DS}}$ igual a $700 \mathrm{mV}$ mostrou que na região de inversão de canal fraco o $\operatorname{HDM}\left(\alpha=135^{\circ}\right)$ apresentou um valor de $g_{m} / I_{D S}$ cerca de $43 \%$ menor que a do CM. Justifica-se esse menor $\mathrm{g}_{\mathrm{m}} / \mathrm{IDS}_{\mathrm{DS}}$ do HMD ( $\alpha$ $=135^{\circ}$ ) por meio da SS dos MOSFETs. Como na região de inversão de canal fraca o $\mathrm{gm} / \mathrm{IDS}$ é inversamente proporcional à SS, para um SS elevada há uma redução do gm/IDS.

Ao analisar as curvas de $\mathrm{IDS}_{\mathrm{DS}}(\mathrm{W} / \mathrm{L})$ em função de $\mathrm{V}_{\mathrm{DS}}$ dos MOSFETs pesquisados, temse que, para um valor de $\mathrm{V}_{\mathrm{GT}}$ igual a $0,7 \mathrm{~V}$ a IDS/(W/L) do $\mathrm{HDM}\left(\alpha=45^{\circ}\right)$, no regime de saturação, apresentou um valor $17,7 \%$ maior que a do CM, enquanto os demais HDMs $(\alpha=$ $\left.90^{\circ} ; 135^{\circ}\right)$ foram menores, devido aos LCE e o PAMDLE, que para o HDM $\left(\alpha=45^{\circ}\right)$ foram capazes de compensar a sua maior razão de aspecto em relação ao CM, proporcionando, dessa forma, um aumento da corrente elétrica. Nos outros $\operatorname{HDMs}\left(\alpha=90^{\circ} ; 135^{\circ}\right)$, apesar de possuírem em suas estruturas os LCE e PAMDLE, não foram capazes de compensar as suas maiores razões de aspecto em relação à do CM.

A partir da derivada das curvas de $\mathrm{IDS}_{\mathrm{DS}}(\mathrm{W} / \mathrm{L})$ em função de $\mathrm{V}_{\mathrm{DS}}$ obteve-se a condutância de dreno, ou condutância de saída, dos MOSFETs. Ao analisar o $\mathrm{g}_{\mathrm{D}} /(\mathrm{W} / \mathrm{L})$ para um $\mathrm{V}_{\mathrm{DS}}$ igual a $700 \mathrm{mV}$ e uma $\mathrm{V}_{\mathrm{GT}}$ igual $700 \mathrm{mV}$ a $\mathrm{g}_{\mathrm{D}} /(\mathrm{W} / \mathrm{L})$ do $\operatorname{HDM}\left(\alpha=45^{\circ}\right)$ é $13,35 \%$ menor que a do 
$\mathrm{CM}$, o gD/(W/L) do HDM $\left(\alpha=90^{\circ}\right)$ foi $26,26 \%$ menor que o do $\mathrm{CM}$ e o HDM $\left(\alpha=135^{\circ}\right)$ teve um valor de $\mathrm{g}_{\mathrm{D}} /(\mathrm{W} / \mathrm{L})$ 19,88\% menor que o medido no CM. Isso ocorre pois os HDMs apresentaram maiores valores de comprimento de canal efetivo e, dessa forma, reduzindo o efeito de modulação de canal.

Uma vez obtido os valores da $\mathrm{gm}_{\mathrm{m}} /(\mathrm{W} / \mathrm{L})$ e da $\mathrm{g}_{\mathrm{D}} /(\mathrm{W} / \mathrm{L})$ dos MOSFETs foi determinado seu valor de ganho de tensão. Analisando os valores de Av constatou-se que os HDMs apresentaram maiores valores de $\mathrm{Av}$, devido à menor $\mathrm{g}_{\mathrm{D}} /(\mathrm{W} / \mathrm{L})$ desses em relação à $\mathrm{g} /(\mathrm{W} / \mathrm{L})$ do CM. O HDM $\left(\alpha=45^{\circ}\right)$ apresentou os maiores valores de Av para os diferentes valores de $\mathrm{V}_{\mathrm{GT}}$, entre os HDMs, isso é justificado por meio dos valores de $\mathrm{g}_{\mathrm{m}} /(\mathrm{W} / \mathrm{L})$ do $\mathrm{HDM}\left(\alpha=45^{\circ}\right)$ que apresentou maiores valores em comparação aos do CM equivalente.

Para poder realizar a análise da distorção harmônica foi utilizado o IFM, por entender que esse método é mais robusto a ruídos em relação aos demais métodos conhecidos na literatura como o de Fourier e a Série de Taylor.

A determinação da THD foi obtida com auxílio do software matemático Mathcad 14.0 e com o grau polinômio de aproximação, utilizado pelo IFM, igual a 5.

A curva da THD em função de $V_{\text {GT }}$ teve seu valor de Vo variando entre $0 \mathrm{~V}$ a $0,7 \mathrm{~V}$, para uma $\mathrm{V}_{\mathrm{DS}}$ igual a $0,7 \mathrm{~V}$ e com amplitude de sinal analógico fixada em $50 \mathrm{mV}$. Nessa primeira análise constatou-se que, para valores de $\mathrm{V}_{\mathrm{GT}}$ menores que $0,55 \mathrm{~V}$, o CM possui a menor THD e para valores de $\mathrm{V}_{\text {GT }}$ maiores que $0,55 \mathrm{~V}$, o HDM $\left(\alpha=45^{\circ}\right)$ apresentou a menor THD entre os MOSFETs. Isso é justificado pela derivada da transcondutância dos MOSFETs e das suas transcondutâncias, nas quais a variação da $g_{m} /(W / L)$ é um fator muito importante para a THD, uma vez que define a forma da curva da THD, isto é, maiores valores da variação de $g_{m} /(W / L)$ menores os valores de THD.

Uma vez que a distorção harmônica é muito relacionada ao Av é feita a normalização da THD pelo Av, na qual constatou-se que, de fato, os HDMs apresentam uma menor THD/Av em toda a região de inversão forte, em especial o $\operatorname{HDM}\left(\alpha=45^{\circ}\right)$, que apresentou até uma redução da THD/Av 6,49 $\mathrm{dB}$ para um $\mathrm{g}_{\mathrm{m}} / \mathrm{IDs}$ igual a $9,4 \mathrm{~V}^{-1}$. Isso se justifica porque, à medida em que os MOSFETs “caminham” para a regime de inversão de canal forte, os HDMs passam a apresentar maiores Av, além disso como a THD/Av passa a ser diretamente proporcional à $\mathrm{g}_{\mathrm{D}} /(\mathrm{W} / \mathrm{L})$, conforme a Equação (39), e os HDMs apresentaram menores valores de $\mathrm{g}_{\mathrm{D}} /(\mathrm{W} / \mathrm{L})$ que o CM, passam a ter um menor valor de THD/Av que o CM.

Ao analisar a última figura de mérito estudada neste projeto de pesquisa, THD/Av em função de $\mathrm{Va}$, em que é diretamente relacionada à capacidade de amplificação de sinais dos 
MOSFET, ao medir a THD/Av em função da amplitude de um sinal senoidal aplicado à porta do dispositivo, com VDs igual a $0,7 \mathrm{~V}$, Va variando entre $0 \mathrm{mV}$ a $100 \mathrm{mV}$ e $\mathrm{V}_{\mathrm{GT}}$ igual a 600 $\mathrm{mV}$, notou-se que o $\operatorname{HDM}\left(\alpha=45^{\circ}\right)$ foi capaz de amplificar sinais senoidais pelo menos 5,81 vezes maiores que o CM equivalente mantendo-se o mesmo nível de linearidade.

Assim conforme mostram os resultados experimentais, o MOSFET de leiaute de porta do tipo Diamante Híbrido (hexagonal híbrido) com ângulo $\alpha$ igual a $45^{\circ}$ pode ser considerado uma alternativa simples e de baixo custo para melhora do desempenho elétrico (Ex: ganho de tensão intrínseco) em paralelo a essa melhora do desempenho elétrico o $\operatorname{HDM}\left(\alpha=45^{\circ}\right)$ apresentou uma THD/Av menor que a do CM equivalente na região de saturação e regime de inversão de canal forte, o que o torna uma interessante opção para aplicações de CIs CMOS analógicos em alta frequência.

Como sugestões de trabalhos futuros, são sugeridas aqui as seguintes possibilidades de pesquisas:

- Estudar a distorção harmônica dos HDMs no regime de inversão de canal moderada e fraca, a fim de verificar se há uma melhora da sua linearidade em comparação ao CM equivalente, para as aplicações onde necessita-se de um alto ganho de tensão.

- Estudar o desempenho elétrico dos HDMs em comparação ao CM em ambientes com radiações ionizantes.

- Estudar o desempenho elétrico dos HDMs em comparação ao CM com a temperatura variando entre $0^{\circ} \mathrm{C}$ a $75^{\circ} \mathrm{C}$.

- Estudar as influências dos efeitos de segunda ordem (Ex: Mobilidade dos portadores) na THD/Av dos HDMs e do CM. 


\section{REFERÊNCIAS}

[1] COLInge, Jean Pierre; COLINGE, Cynthia A., Physics of Semiconductor Devices, Springer; $2^{\text {nd }}$ edition, 2002.

[2] COLINGE, Jean Pierre, Silicon- On-Insulator Technology: Materials to VLSI, Springer; $2^{\text {nd }}$ edition, 1997.

[3] SCHRODER, D. K. Semiconductor Material and Device Characterization. $3^{\text {rd }}$ edition. Hoboken: IEEE, 2006.

[4] GROENEWOLD, G.; LUBBERS, W. J.; Systematic Distortion Analysis for MOSFET Integrators with Use of a New MOSFET Model, IEEE Transactions on Circuits and Systems - Part II: Analog and Digital Signal Processing, v. 41, no. 9, p. 569-580, 1994.

[5] V. KILCHYTSKA ; B. KAZEMI ESFEH ; C. GIMENO ; B. PARVAIS ; N. PLANES ; M. HAOND ; J.-P. RASKIN ; D. FLANDRE; Comparative study of non-linearities in $28 \mathbf{~ n m}$ node FDSOI and Bulk MOSFETs, Joint International EUROSOI Workshop and International Conference on Ultimate Integration on Silicon (EUROSOI-ULIS), 2017.

[6] DORIA, R. T. Estudo da linearidade em transistores SOI de porta dupla com estrutura de canal gradual. 2007. 144f. Dissertação (Mestrado em Dispositivos Eletrônicos) - Centro Universitário FEI, São Bernardo do Campo, 2007.

[7] DORIA, RODRIGO T.; PAVANELLO, MARCELO A., TREVISOLI, RENAN D.; SOUZA, MICHELLY; LEE, CHI-WOO; FERAIN, ISABELLE; COLINGE, JEAN-PIERRE; Analog operation temperature dependence of nMOS junctionless transistors focusing on harmonic distortion, Journal of Integrated Circuits and Systems, v. 6, p. 114-121, 2011.

[8] DANTAS, Leandro P. Estudo da Distorção Harmônica em Transistores de Porta Circular Usando Tecnologia SOI CMOS SubMicrométrica de 0,13 $\boldsymbol{\mu m}$. 2008. $146 \mathrm{~F}$. Dissertação (Mestrado em Dispositivos Eletrônicos) - Centro Universitário FEI, São Bernardo do Campo, 2008.

[9] GIMENEZ, S. P., Diamond MOSFET: An innovative layout to improve performance of ICs, Solid-State Electronics, vol. 54, p. 1690-1699, 2010.

[10] GIMENEZ, S. P. ; GALEMBECK, E. H. S. ; FLANDRE, D. ; RENAUX, C., Diamond leiaute style impact on SOI MOSFET in high temperature environment. Microelectronics and Reliability, v. 55, p. 783/MR11492-788, 2015.

[11] SEIXAS, L.E.; FINCO, S. ; SILVEIRA, M. A. G. ; MEDINA, N.; GIMENEZ, S. P., Study of proton radiation effects among diamond and rectangular gate MOSFET layouts. Materials Research Express, vol. 4, p. 015901, 2017.

[12] FINO, L. N. S. ; NETO, E. D. ; SILVEIRA, M. A. G. ; RENAUX, C. ; FLANDRE, D. ; GIMENEZ, S. P., Boosting the total ionizing dose tolerance of digital switches by using OCTO SOI MOSFET, Semiconductor Science and Technology, v. 30, p. 105017-12p, 2015. 
[13] GIMENEZ, S. P. ; CORREIA, M. M. ; NETO, E. D. ; SILVA, C. R., An Innovative Ellipsoidal Layout Style to Further Boost the Electrical Performance of MOSFETs, IEEE Electron Device Letters, v. 36, p. 705-707, 2015.

[14] SONNENBERG, Victor; NICOLETT, Aparecido S. Transistores MOSFETs fabricados na tecnologia de silício sobre isolante - SOIMOSFET: a caminho do futuro. Boletim Técnico da Faculdade de Tecnologia de São Paulo, BT14, 14-23, Ago/2003.

[15] ZIMPECK, Alexandra L. ;MEINHARDT, Cristina e BUTZEN, Paulo F.; Análise do comportamento de portas lógicas CMOS com falhas Stuck - On em nanotecnologias. ICCEEg, vol. 1, nº 7, pp. 1-10, 2014.

[16] MARTINO, João A.; PAVANELlO, Marcelo A.; VERDONCK, Patrick B. Caracterização Elétrica de Tecnologia e Dispositivos, Pioneira Thomson Learning, São Paulo, Brazil, 2003.

[17] TERADA, K.; NISHIYAMA, K.; HATANAKA, K.; Comparison of MOSFET threshold-voltage extraction methods, Solid-State Electronics, vol. 45, p. 35-40, jan. 2001.

[18] CONDE, O. A., SÁNCHEZ, F. J. G., LIOU, J. J., CERDEIRA, A., EESTRADA, M., YUE $Y$. A review of recent MOSFET threshold voltage extraction methods, Microelectronics Reliability, v. 42, p. 583-596, 2002.

[19] M.B. Machado, O.F. Siebel, M.C. Schneider e C. Galup-Montoro. MOSFET Threshhold Voltage: Defenition, Extraction and Applications. NSTI-Nanotech 2011, Vol. 2, 2011.

[20] FLANDRE, D.; SILVEIRA, F. A $\mathbf{g}_{\mathbf{m}} / \mathbf{I}_{\mathbf{D}}$ Based Methodology for the Design of CMOS Analog Circuits and Its Application to the Synthesis of a Silicon-on-Insulator Micropower OTA. IEEE Journal of solid-state circuits, Vol. 31, n.9, set. 1996.

[21] LANGEVELDE, R. Van.; KLAASSEN, F.M.; Accurate Drain Conductance Modeling for Distortion Analysis in MOSFETs, IEEE Transactions on Electron Devices, v. 44, no. 11, p. 2044-2052, 1997.

[22] SANSEN, Willy, Distortion in Elementary Transistor Circuits, IEEE Transactions on Circuits and Systems - Part II: Analog and Digital Signal Processing, v. 46, no. 3, p. 315-326, 1999.

[23] WAMBACQ, P.; SANSEN, W.; Distortion Analysis of Analog Integrated Circuits, Dordecht: Kluwer Academic Publishers, 1998.

[24] HSU, H. P.; Análise de Fourier, Rio de Janeiro: Livros Técnicos e Científicos Editora, 1972.

[25] CERDEIRA, A.; ALEMÁN, M. A.; ESTRADA, M.; FLANDRE, D.; PARVAIS, B.; PICUN, G.; The Integral Function Method: A New Method to Determine the Non-Linear Harmonic Distortion, In: SBMicro $2003-18^{\text {th }}$ International Symposium on Microelectronics Technology and Devices, p. 131-146, São Paulo, 2003. 
[26] CERDEIRA, A.; ALEMÁN, M. A.; ESTRADA, M.; FLANDRE, D.; Integral Function Method for Determination of Nonlinear Harmonic Distortion, Solid-State Electronics, v. 48, n. 12, p. 2226-2234, 2004.

[27] CERDEIRA, A.; ESTRADA, M; QUINTERO, R; FLANDRE, D; ORTIZ-CONDE, A; GARCÍA SÁNCHEZ, F. J.; Generalization of the integral function method to evaluate distortion in SOI FD MOSFET. In: Proceedings of the 23 International Conference on Microelectronics, v. 2. p. 443-446, Nis, Yugoslavia, 2002.

[28] CERDEIRA, A.; ESTRADA, M.; Mathematical Basis of the Expressions Used by the Integral Function Method for the Determination of Nonlinear Harmonic Distortion in Devices and Circuits; In: Proceedings of the $7^{\text {th }}$ International Conference on Solid State and IC Technology, p.1143-1146, Bejing, China, 2004.

[29] GIMENEZ, S. P.; BELlodi, M. Diamond SOI MOSFET: A New Drain and Source/Channel Interface Layout to Improve Drain Current. Fifth Workshop of the Thematic Network on Silicon on Insulator Technology, Devices and Circuits, Göteborg, January 2009.

[30] ALATI, D. M.; GIMENEZ, S. P. Comparação entre o Transistor SOI nMOSFET Diamante e seu Equivalente Convencional. Anais do XI Simpósio de Iniciação Científica e Tecnológica - XI SICT, São Paulo, 07 Outubro 2009. 76.

[31] GIMENEZ, S. P.; CLASER, R.; ALATI, D. M. Using Diamond SOI nMOSFETs to Improve the Frequency Response of the Analog Integrated Circuits. Seventh Workshop of the Thematic Network on Silicon on Insulator Technology, Devices and Circuits, Granada, January 2011.

[32] ALATI, D. M.; GIMENEZ, S. P. Estudo Comparativo entre o SOI nMOSFET Diamante e Convencional, Utilizando o Simulador Sentaurus Device da Synopsys. XVII Simpósio Internacional de Iniciação Científica USP (XVII SIICUSP), São Carlos, 09 Novembro 2009.

[33] GIMENEZ, S. P. ; RENAUX, C. ; LEONI, R. D. ; FLANDRE, D., Using diamond layout style to boost MOSFET frequency response of analogue IC, Electronics Letters, vol. 50, p. 398-400, 2014.

[34] GIMENEZ, S. P.; ALATI, D. M.; SILVEIRA, M. A. G.; MELO W.; SEIXAS L. E.; MEDINA N. H.; ADDED N.; TABACNICKS M., The Protons Radiation-Robustness Of Integrated Circuits By Using The Diamond Leiaute Style, In: RADECS 2012, Biarritz, France, 2012.

[35] E. A. VITTOZ, Low-power low-voltage limitations and prospects in analog design, in Proc. Workshop Advances in Analog Circuit Design, Eindhoven, Mar. 1994.

[36] PARVAIS, B.; CERDEIRA, A.; SCHREURS, D.; RASKIN, J. P.; Harmonic Distortion Characterization of SOI MOSFETs, In: $11^{\text {th }}$ GAAS Symposium, p. 357-360, Munique, 2003.

[37] CERDEIRA, A.; AlEMÁN, M. A.; PAVANELlO, M. A.; MARTINO J. A.; VANCAILLIE, L.; FLANDRE, D.; Advantages of the Graded-Channel SOI FD MOSFET 
for Application as a Quasi-Linear Resistor, IEEE Transactions on Electron Devices, v. 52, n. 5 , p. $967-972,2005$.

[38] GLENN, Knoll F., Radiation Detection and Measurement. New York Wiley, 2000.

[39] HUGHES, R. C., Charge carrier transport phenomena in amorphous $\mathrm{SiO}_{2}$ : Direct measurement of mobility and carrier lifetime, Phys. Rev. Lett.,vol. 30, 1973.

[40] LUTZ, G., Semiconductor Radiation Detectors. Springer, 1999. 
APÊNDICE A - ARTIGOS PUBLICADOS 
- Improvement the harmonic distortion using the Diamond leiaute Style for MOSFET

SEMINATEC 2018 (Semiconductors and Micro \& Nano Technology) - JUNE 2018 http://fei.edu.br/seminatec/paper_8.pdf

- Improvement of the Harmonic Distortion by Using Diamond MOSFET

Conference: $201833^{\text {rd }}$ Symposium on Microelectronics Technology and Devices (SBMicro) - SBMICRO 2018 - AUGUST 2018

https://ieeexplore.ieee.org/document/8511499/metrics\#metrics 